\title{
An Elliptic Hypergeometric Function Approach to Branching Rules
}

\author{
Chul-hee LEE ${ }^{\dagger}$, Eric M. RAINS $\ddagger$ and S. Ole WARNAAR $\S$ \\ $\dagger$ School of Mathematics, Korea Institute for Advanced Study, Seoul 02455, Korea \\ E-mail: chlee@kias.re.kr \\ ¥Department of Mathematics, California Institute of Technology, Pasadena, CA 91125, USA \\ E-mail: rains@caltech.edu \\ $\S$ School of Mathematics and Physics, The University of Queensland, \\ Brisbane, QLD 4072, Australia \\ E-mail: o.warnaar@maths.uq.edu.au
}

Received July 08, 2020, in final form December 09, 2020; Published online December 23, 2020

https://doi.org/10.3842/SIGMA.2020.142

\begin{abstract}
We prove Macdonald-type deformations of a number of well-known classical branching rules by employing identities for elliptic hypergeometric integrals and series. We also propose some conjectural branching rules and allied conjectures exhibiting a novel type of vanishing behaviour involving partitions with empty 2 -cores.
\end{abstract}

Key words: branching formulas; elliptic hypergeometric series; elliptic Selberg integrals; interpolation functions; Koornwinder polynomials; Littlewood identities; Macdonald polynomials

2020 Mathematics Subject Classification: 05E05; 05E10; 20C33; 33D05; 33D52; 33D67

\section{Branching rules}

Let $\mathfrak{g}$ be a semisimple complex Lie algebra of rank $r$, with fundamental weights $\omega_{1}, \ldots, \omega_{r}$. Denote by $V(\lambda ; \mathfrak{g})$ the irreducible $\mathfrak{g}$-module of highest weight $\lambda=\sum_{i=1}^{r} \lambda_{i} \omega_{i}, \lambda_{i} \in \mathbb{Z}_{\geqslant 0}$. For $\mathfrak{h}$ a subalgebra of $\mathfrak{g}$, let $\left.V(\lambda ; \mathfrak{g})\right|_{\mathfrak{h}}$ be the restriction of $V(\lambda ; \mathfrak{g})$ to $\mathfrak{h}$. Generally, the $\mathfrak{h}$-module $\left.V(\lambda ; \mathfrak{g})\right|_{\mathfrak{h}}$ is not irreducible, and the branching problem refers to the problem of determining the multiplicities $\operatorname{mult}_{\lambda}(\mu)$ in

$$
\left.V(\lambda ; \mathfrak{g})\right|_{\mathfrak{h}}=\bigoplus_{\mu} \operatorname{mult}_{\lambda}(\mu) V(\mu ; \mathfrak{h})
$$

where $\mu$ runs over the weights indexing the irreducible $\mathfrak{h}$-modules, see, e.g., $[18,19,25]$. When $\operatorname{mult}_{\lambda}(\mu) \leqslant 1$ for all $\mu$ we say that the branching rule (1.1) is multiplicity free.

Proposition 1.1. Let $\mathfrak{g}=\operatorname{sl}(2 n, \mathbb{C}), \mathfrak{h}=\operatorname{sp}(2 n, \mathbb{C})$ with canonical embedding $\mathfrak{h} \hookrightarrow \mathfrak{g}$. For $m, r, p$ integers such that $1 \leqslant r \leqslant n$ and $0 \leqslant p \leqslant m$,

$$
\left.V\left(p \omega_{r-1}+(m-p) \omega_{r} ; \mathfrak{g}\right)\right|_{\mathfrak{h}}=\bigoplus_{\begin{array}{c}
m_{0}, \ldots, m_{r} \geqslant 0 \\
m_{0}+m_{1}+\cdots+m_{r}=m \\
m_{r-1}+m_{r-3}+\cdots=p
\end{array}} V\left(m_{1} \omega_{1}+\cdots+m_{r} \omega_{r} ; \mathfrak{h}\right) .
$$

This paper is a contribution to the Special Issue on Elliptic Integrable Systems, Special Functions and Quantum Field Theory. The full collection is available at https://www.emis.de/journals/SIGMA/elliptic-integrablesystems.html 
For $p=0$ this is Proctor's branching rule [34, Lemma 4] (see also [31, Theorem 2.6]), and for general $p$ it is equivalent to the following combinatorial identity of Krattenthaler [21, equation (3.3)]. Given a partition $\lambda$, let $\lambda^{\prime}$ be its conjugate and $l^{\circ}(\lambda)$ the number of odd parts of $\lambda$. Moreover, for $\lambda \subset\left(m^{r}\right)$ (i.e., $\lambda=\left(\lambda_{1}, \ldots, \lambda_{r}\right)$ such that $\left.0 \leqslant \lambda_{r} \leqslant \cdots \leqslant \lambda_{1} \leqslant m\right)$, let $\left(m^{r}\right)-\lambda$ be shorthand for the complement of $\lambda$ with respect to $\left(m^{r}\right)$ (i.e., $\left(m^{r}\right)-\lambda=\left(m-\lambda_{r}, \ldots, m-\lambda_{1}\right)$. Then

$$
s_{\left(m^{r-1}, m-p\right)}\left(x_{1}^{ \pm}, \ldots, x_{n}^{ \pm}\right)=\sum_{\substack{\lambda \subset\left(m^{r}\right) \\ l^{\circ}\left(\lambda^{\prime}\right)=p}} \operatorname{sp}_{2 n,\left(m^{r}\right)-\lambda}\left(x_{1}, \ldots, x_{n}\right) .
$$

Here $s_{\lambda}$ and $\mathrm{sp}_{2 n, \lambda}$ are the Schur function and symplectic Schur function indexed by the partition $\lambda$ respectively $[25,26]$, and $f\left(x_{1}^{ \pm}, \ldots, x_{n}^{ \pm}\right):=f\left(x_{1}, x_{1}^{-1}, \ldots, x_{n}, x_{n}^{-1}\right)$. For a more gen-

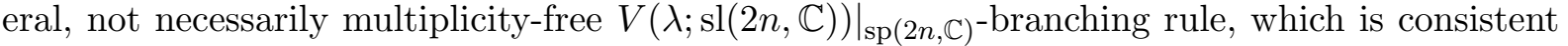
with (1.2) and which is expressed in terms of Littelmann paths, we refer to [29, 47].

Krattenthaler's Schur function identity (1.2) follows from a more general identity of his for the universal symplectic characters $\operatorname{sp}_{\lambda}=\operatorname{sp}_{\lambda}\left(x_{1}, x_{2}, \ldots\right)$ introduced by Koike and Terada [19]. The latter specialise to the symplectic Schur functions as

$$
\operatorname{sp}_{\lambda}\left(x_{1}^{ \pm}, \ldots, x_{n}^{ \pm}, 0,0, \ldots\right)= \begin{cases}\operatorname{sp}_{2 n, \lambda}\left(x_{1}, x_{2}, \ldots, x_{n}\right) & \text { if } l(\lambda) \leqslant n \\ 0 & \text { otherwise }\end{cases}
$$

and the lift of (1.2) to universal characters is given by [21, equation (3.1)]

$$
s_{\left(m^{r-1}, m-p\right)}=\sum_{\substack{\lambda \subset\left(m^{r}\right) \\ l^{\circ}\left(\lambda^{\prime}\right)=p}} \operatorname{sp}_{\left(m^{r}\right)-\lambda} .
$$

The branching problem is an important problem in representation theory, algebraic combinatorics and orthogonal polynomials on root systems, see e.g., [8, 9, 15, 16, 18, 19, 21, 22, 23, 24, $25,26,29,31,34,35,47]$. In this paper we are interested in $q, t$-analogues of multiplicity-free branching rules such as (1.2) and (1.4). We prove non-trivial new examples of what appears to be a very general phenomenon: the natural $q, t$-analogues of multiplicity-free formulas arising in the representation theory of the classical groups, be it branching formulas, tensor product decompositions or other types of multiplicity formulas, are usually 'nice'. More precisely, the $q, t$-analogue of a multiplicity 1 appears to almost always factor as ratio of products of binomials of the form $1-q^{k} t^{\ell}$. For some representative examples of this phenomenon, see, e.g., $[7,26,35,41,42]$.

The natural $q, t$-analogues of the Schur functions $s_{\lambda}$ are the Macdonald polynomials $P_{\lambda}(q, t)$ [26]. Similarly, the natural analogues of the symplectic Schur function $\mathrm{sp}_{2 n, \lambda}$ are the $\mathrm{C}_{n}$ Macdonald polynomials [27]

$$
P_{\lambda}^{\left(\mathrm{C}_{n}, \mathrm{~B}_{n}\right)}(q, t, s) \quad \text { and } \quad P_{\lambda}^{\left(\mathrm{C}_{n}, \mathrm{C}_{n}\right)}(q, t, s) .
$$

It will be convenient to view these two families of $\left(\mathrm{BC}_{n}\right.$ symmetric Laurent) polynomials as special instances of the Koornwinder polynomials $K_{\lambda}(q, t ; \underline{t})=K_{\lambda}\left(q, t ; t_{0}, t_{1}, t_{2}, t_{3}\right)$, [20]. Specifically, see, e.g., [42],

$$
\begin{aligned}
& P_{\lambda}^{\left(\mathrm{C}_{n}, \mathrm{~B}_{n}\right)}(q, t, s)=K_{\lambda}\left(q, t ; s^{1 / 2},-s^{1 / 2}, q^{1 / 2},-q^{1 / 2}\right), \\
& P_{\lambda}^{\left(\mathrm{C}_{n}, \mathrm{C}_{n}\right)}(q, t, s)=K_{\lambda}\left(q, t ; s^{1 / 2},-s^{1 / 2},(q s)^{1 / 2},-(q s)^{1 / 2}\right) .
\end{aligned}
$$


In the following we will also need the $\mathrm{B}_{n}$ Macdonald polynomial

$$
P_{\lambda}^{\left(\mathrm{B}_{n}, \mathrm{C}_{n}\right)}(q, t, s)=K_{\lambda}\left(q, t ;-1,-q^{1 / 2}, s, s q^{1 / 2}\right),
$$

where we restrict ourselves to partitions $\lambda$. $^{1}$

Finally, in view of (1.7), the $q, t$-analogues of the universal symplectic characters are specialisations of the lifted Koornwinder polynomials $\tilde{K}_{\lambda}(q, t, T ; \underline{t})$, [35]. These symmetric functions, which contain the extra parameter $T$, specialise to the Koornwinder polynomials as

$$
\tilde{K}_{\lambda}\left(x_{1}^{ \pm}, \ldots, x_{n}^{ \pm}, 0,0, \ldots ; q, t, t^{n} ; \underline{t}\right)= \begin{cases}K_{\lambda}\left(x_{1}, \ldots, x_{n} ; q, t ; \underline{t}\right) & \text { if } l(\lambda) \leqslant n \\ 0 & \text { otherwise. }\end{cases}
$$

In fact, the lifted Koornwinder polynomials (which are not polynomials) are the unique symmetric functions such that (1.7) holds, so that the above may serve as the definition of the $\tilde{K}_{\lambda}$.

The first main result of this paper is the following triple of $q, t$-branching rules. Let $C_{\lambda}^{0}(z ; q, t)$, $C_{\lambda}^{-}(z ; q, t)$ and $C_{\lambda}^{+}(z ; q, t)$ be the standard three families of $q, t$-shifted factorials indexed by partitions (see (2.7) and (2.15) below), and, for $\lambda$ a partition, let $2 \lambda:=\left(2 \lambda_{1}, 2 \lambda_{2}, \ldots\right)$ and $\lambda^{2}:=\left(\lambda_{1}, \lambda_{1}, \lambda_{2}, \lambda_{2}, \ldots\right)$.

Theorem 1.2. For $m, r$ nonnegative integers,

$$
\begin{aligned}
& P_{\left(m^{r}\right)}(q, t)=\sum_{\substack{\lambda \subset\left(m^{r}\right) \\
\lambda^{\prime} \text { even }}} c_{\lambda}\left(q^{-m}, t^{r} / T ; q, t\right) \tilde{K}_{\left(m^{r}\right)-\lambda}\left(q, t, T ; t^{1 / 2},-t^{1 / 2},(q t)^{1 / 2},-(q t)^{1 / 2}\right), \\
& P_{\left(m^{r}\right)}(q, t)=\sum_{\substack{\lambda \subset\left(m^{r}\right) \\
\lambda \text { even }}} d_{\lambda}\left(q^{-m}, t^{r} / T ; q, t\right) \tilde{K}_{\left(m^{r}\right)-\lambda}\left(q, t, T ; 1,-1, t^{1 / 2},-t^{1 / 2}\right), \\
& P_{\left(m^{r}\right)}\left(q^{2}, t^{2}\right)=\sum_{\lambda \subset\left(m^{r}\right)} e_{\lambda}\left(q^{-m}, t^{r} / T ; q, t\right) \tilde{K}_{\left(m^{r}\right)-\lambda}\left(q^{2}, t^{2}, T^{2} ;-1,-q,-t,-q t\right),
\end{aligned}
$$

where

$$
\begin{aligned}
& c_{\lambda^{2}}(w, z ; q, t):=\left(\frac{q}{t}\right)^{|\lambda|} \frac{C_{\lambda^{2}}^{0}(w ; q, t)}{C_{\lambda^{2}}^{0}(q w / t ; q, t)} \frac{C_{\lambda}^{-}\left(t ; q, t^{2}\right)}{C_{\lambda}^{-}\left(q ; q, t^{2}\right)} \frac{C_{\lambda}^{+}\left(q w^{2} z^{2} / t^{4} ; q, t^{2}\right)}{C_{\lambda}^{+}\left(w^{2} z^{2} / t^{3} ; q, t^{2}\right)}, \\
& d_{2 \lambda}(w, z ; q, t):=\left(\frac{q}{t}\right)^{|\lambda|} \frac{C_{2 \lambda}^{0}(w ; q, t)}{C_{2 \lambda}^{0}(q w / t ; q, t)} \frac{C_{\lambda}^{-}\left(q t ; q^{2}, t\right)}{C_{\lambda}^{-}\left(q^{2} ; q^{2}, t\right)} \frac{C_{\lambda}^{+}\left(q^{2} w^{2} z^{2} / t^{2} ; q^{2}, t\right)}{C_{\lambda}^{+}\left(q w^{2} z^{2} / t ; q^{2}, t\right)}, \\
& e_{\lambda}(w, z ; q, t):=\left(\frac{q}{t}\right)^{|\lambda|} \frac{C_{\lambda}^{0}\left(w^{2} ; q^{2}, t^{2}\right)}{C_{\lambda}^{0}\left(q^{2} w^{2} / t^{2} ; q^{2}, t^{2}\right)} \frac{C_{\lambda}^{-}(-t ; q, t)}{C_{\lambda}^{-}(q ; q, t)} \frac{C_{\lambda}^{+}\left(q w^{2} z^{2} / t^{2} ; q, t\right)}{C_{\lambda}^{+}\left(-w^{2} z^{2} / t ; q, t\right)} .
\end{aligned}
$$

To highlight the combinatorial and factorised nature of the coefficients in (1.9) we have rewritten each one of them in terms of the ordinary (or type-A) arm-(co)lengths and leg-(co)lengths of the squares $s \in \lambda$ as well as their type-C analogues (see Section 2.1):

$$
\begin{aligned}
c_{\lambda^{2}}(w, z ; q, t)=\prod_{s \in \lambda} & \left(\frac{q\left(1-w q^{a^{\prime}(s)} t^{-2 l^{\prime}(s)}\right)\left(1-w q^{a^{\prime}(s)} t^{-2 l^{\prime}(s)-1}\right)}{t\left(1-w q^{a^{\prime}(s)+1} t^{-2 l^{\prime}(s)-1}\right)\left(1-w q^{a^{\prime}(s)+1} t^{-2 l^{\prime}(s)-2}\right)}\right. \\
& \left.\times \frac{\left(1-q^{a(s)} t^{2 l(s)+1}\right)\left(1-w^{2} z^{2} q^{\hat{a}(s)+1} t^{-2 \hat{l}(s)-2}\right)}{\left(1-q^{a(s)+1} t^{2 l(s)}\right)\left(1-w^{2} z^{2} q^{\hat{a}(s)} t^{-2 \hat{l}(s)-1}\right)}\right),
\end{aligned}
$$

\footnotetext{
${ }^{1}$ The Macdonald polynomials $P_{\lambda}^{\left(\mathrm{B}_{n}, \mathrm{C}_{n}\right)}\left(q, t, t_{2}\right)$ may also be defined for half-partitions $\lambda=\left(\lambda_{1}, \ldots, \lambda_{n}\right)$ where $\lambda_{1} \geqslant \cdots \geqslant \lambda_{n} \geqslant 0$ and $\lambda_{i} \in \frac{1}{2}+\mathbb{Z}$, in which case the right-hand side of (1.6) needs to be slightly modified [42].
} 


$$
\begin{aligned}
d_{2 \lambda}(w, z ; q, t)=\prod_{s \in \lambda} & \left(\frac{q\left(1-w q^{2 a^{\prime}(s)} t^{-l^{\prime}(s)}\right)\left(1-w q^{2 a^{\prime}(s)+1} t^{-l^{\prime}(s)}\right)}{t\left(1-w q^{2 a^{\prime}(s)+1} t^{-l^{\prime}(s)-1}\right)\left(1-w q^{2 a^{\prime}(s)+2} t^{-l^{\prime}(s)-1}\right)}\right. \\
& \left.\times \frac{\left(1-q^{2 a(s)+1} t^{l(s)+1}\right)\left(1-w^{2} z^{2} q^{2 \hat{a}(s)+2} t^{-\hat{l}(s)-1}\right)}{\left(1-q^{2 a(s)+2} t^{l(s)}\right)\left(1-w^{2} z^{2} q^{2 \hat{a}(s)+1} t^{-\hat{l}(s)}\right)}\right),
\end{aligned}
$$

and

$$
e_{\lambda}(w, z ; q, t)=\prod_{s \in \lambda} \frac{q\left(1-w^{2} q^{2 a^{\prime}(s)} t^{-2 l^{\prime}(s)}\right)\left(1+q^{a(s)} t^{l(s)+1}\right)\left(1-w^{2} z^{2} q^{\hat{a}(s)+1} t^{-\hat{l}(s)-1}\right)}{t\left(1-w^{2} q^{2 a^{\prime}(s)+2} t^{-2 l^{\prime}(s)-2}\right)\left(1-q^{a(s)+1} t^{l(s)}\right)\left(1+w^{2} z^{2} q^{\hat{a}(s)} t^{-\hat{l}(s)}\right)} .
$$

For $t=q,(1.8 \mathrm{a})$ and (1.8b) simplify to the $p=0$ instances of (1.4) and [21, equation (3.2)]

$$
s_{\left(m^{r-p},(m-1)^{p}\right)}=\sum_{\substack{\lambda \subset\left(m^{r}\right) \\ l^{\circ}(\lambda)=p}} \mathrm{O}_{\left(m^{r}\right)-\lambda}
$$

respectively. Here $o_{\lambda}$ is a universal orthogonal character (see Section 3 for details). Finally, (1.8c) for $t=-q$ yields

$$
s_{\left(m^{r}\right)}=\sum_{\lambda \subset\left(m^{r}\right)}(-1)^{|\lambda|} \mathrm{SO}_{\left(m^{r}\right)-\lambda}
$$

where $\mathrm{so}_{\lambda}$ is a universal special orthogonal character. The fact that the parameters $q$ and $T$ (the latter only occurs on the right-hand side of (1.8a)-(1.8c)) is a consequence of Lemma 4.1 proved in Section 4.3.

By (1.5)-(1.7), Theorem 1.2 has the following corollary.

Corollary 1.3. Let $m, r, n$ be nonnegative integers such that $r \leqslant n$, and set

$$
x:=\left(x_{1}, \ldots, x_{n}\right) \quad \text { and } \quad x^{ \pm}:=\left(x_{1}, x_{1}^{-1}, \ldots, x_{n}, x_{n}^{-1}\right) .
$$

Then

$$
\begin{aligned}
& P_{\left(m^{r}\right)}\left(x^{ \pm} ; q, t\right)=\sum_{\substack{\lambda \subset\left(m^{r}\right) \\
\lambda^{\prime} \text { even }}} c_{\lambda}\left(q^{-m}, t^{-(n-r)} ; q, t\right) P_{\left(m^{r}\right)-\lambda}^{\left(\mathrm{C}_{n}, \mathrm{C}_{n}\right)}(x ; q, t, t), \\
& P_{\left(m^{r}\right)}\left(x^{ \pm} ; q, t\right)=\sum_{\substack{\lambda \subset\left(m^{r}\right) \\
\lambda \text { even }}} d_{\lambda}\left(q^{-m}, t^{-(n-r)} ; q, t\right) K_{\left(m^{r}\right)-\lambda}\left(x ; q, t ; 1,-1, t^{1 / 2},-t^{1 / 2}\right), \\
& P_{\left(m^{r}\right)}\left(x^{ \pm} ; q^{2}, t^{2}\right)=\sum_{\lambda \subset\left(m^{r}\right)} e_{\lambda}\left(q^{-m}, t^{-(n-r)} ; q, t\right) P_{\left(m^{r}\right)-\lambda}^{\left(\mathrm{B}_{n}, \mathrm{C}_{n}\right)}\left(x ; q^{2}, t^{2},-t\right) .
\end{aligned}
$$

For $t=q,(1.12 \mathrm{a})$ and (1.12b) simplify to the $p=0$ case of (1.2) and its dual [21, equation $(3.5) ; p=0]$

$$
s_{\left(m^{r}\right)}\left(x_{1}^{ \pm}, \ldots, x_{n}^{ \pm}\right)=\sum_{\substack{\lambda \subset\left(m^{r}\right) \\ \lambda \text { even }}} \mathrm{o}_{2 n,\left(m^{r}\right)-\lambda}\left(x_{1}, \ldots, x_{n}\right),
$$

respectively.

We conjecture one more branching rule of type $\left(\mathrm{C}_{n}, \mathrm{~B}_{n}\right)$. For the notation used in this conjecture we refer to Sections 2.1 and 2.2. 
Conjecture 1.4. For $m, r$ nonnegative integers,

$$
P_{\left(m^{r}\right)}(q, t)=\sum_{\substack{\lambda \subset\left(m^{r}\right) \\ 2-\operatorname{core}(\lambda)=0}} f_{\lambda}\left(q^{-m}, q^{r} / T ; q, t\right) \tilde{K}_{\left(m^{r}\right)-\lambda}\left(q, t, T ; \pm q^{1 / 2}, \pm t^{1 / 2}\right)
$$

and for $m, r, n$ nonnegative integers such that $r \leqslant n$,

$$
P_{\left(m^{r}\right)}\left(x^{ \pm} ; q, t\right)=\sum_{\substack{\lambda \subset\left(m^{r}\right) \\ 2-\operatorname{core}(\lambda)=0}} f_{\lambda}\left(q^{-m}, q^{-(n-r)} ; q, t\right) P_{\left(m^{r}\right)-\lambda}^{\left(\mathrm{C}_{n}, \mathrm{~B}_{n}\right)}(x ; q, t, t),
$$

where

$$
\begin{aligned}
f_{\lambda}(w, z ; q, t):= & \left(\frac{q}{t}\right)^{|\lambda| / 2} q^{2 \hat{n}^{\mathrm{o}}\left(\lambda^{\prime}\right)-2 \hat{n}^{\mathrm{e}}\left(\lambda^{\prime}\right)} t^{n^{\mathrm{e}}(\lambda)-n^{\mathrm{o}}(\lambda)} \\
& \times \frac{C_{\lambda}^{0}(w ; q, t)}{C_{\lambda}^{0}(q w / t ; q, t)} \frac{C_{\lambda}^{-, \mathrm{e}}(t ; q, t)}{C_{\lambda}^{-, \mathrm{o}}(q ; q, t)} \frac{C_{\lambda}^{+, \mathrm{e}}\left(q w^{2} z^{2} / t^{2} ; q, t\right)}{C_{\lambda}^{+, \mathrm{o}}\left(w^{2} z^{2} / t ; q, t\right)} .
\end{aligned}
$$

The remainder of the paper is organised as follows. The next section covers some introductory material on partitions, various kinds of shifted factorial, and elliptic hypergeometric series. Then, in Section 3, we introduce some of the standard bases of the ring of symmetric functions and discuss the various types of classical Schur functions and classical branching rules. The final introductory section is Section 4, in which we survey material from Macdonald-Koornwinder theory, including the elliptic generalisation of this theory. In Sections 5-7 we prove a number of new results needed for our proof of Theorem 1.2 and Corollary 1.3, which is presented in Section 6.2. These new results include the evaluations of two quadratic elliptic beta integrals over elliptic interpolation functions (Theorems 5.1 and 5.4), their corresponding discrete analogues (Corollaries 5.3 and 5.6), a formula for the transition coefficients between Okounkov's $\mathrm{BC}_{n}$-symmetric Macdonald interpolation polynomials and ordinary Macdonald polynomials (Theorem 6.3), and a number of quadratic summations for a new type of elliptic hypergeometric series (Theorems 7.3 and 7.5). Finally, in Section 9 we propose a number of conjectures in the spirit of Conjecture 1.4. As a simple example, we conjecture the new Littlewood-type identity

$$
\sum_{\lambda} \kappa_{\lambda}^{(1)}(q, t) P_{\lambda}(x ; q, t)=\prod_{i \geqslant 1} \frac{\left(t x_{i}^{2} ; q^{2}\right)_{\infty}}{\left(x_{i}^{2} ; q^{2}\right)_{\infty}} \prod_{i<j} \frac{\left(t x_{i} x_{j} ; q\right)_{\infty}}{\left(x_{i} x_{j} ; q\right)_{\infty}},
$$

where $(a ; q)_{\infty}:=(1-a)(1-a q)\left(1-a q^{2}\right) \cdots$,

$$
\kappa_{\lambda}^{(1)}(q, t):= \begin{cases}\frac{\prod_{(i, j) \in \lambda}^{\mathrm{e}}\left(q^{1-\lambda_{i}} t^{i-1}-q^{1-j} t^{\lambda_{j}^{\prime}}\right)}{\prod_{(i, j) \in \lambda}^{\mathrm{o}}\left(q^{1-\lambda_{i}} t^{i-1}-q^{2-j} t^{\lambda_{j}^{\prime}-1}\right)} & \text { if 2-core }(\lambda)=0, \\ 0 & \text { otherwise, }\end{cases}
$$

and

$$
\prod_{(i, j) \in \lambda}^{\mathrm{e} / \mathrm{o}} f_{i j}:=\prod_{\substack{s=(i, j) \in \lambda \\ a(s)+l(s) \text { even/odd }}} f_{i j}
$$

To answer a question by the referee regarding the connection between the branching rule (1.12a) and two conjectural branching rules by Hoshino and Shiraishi from [15] we have added a postsript on pages $48-50$. 


\section{Preliminaries}

\subsection{Partitions}

A partition $\lambda=\left(\lambda_{1}, \lambda_{2}, \ldots\right)$ is a sequence of weakly decreasing nonnegative integers such that $|\lambda|:=\lambda_{1}+\lambda_{2}+\cdots$ is finite. If $|\lambda|=n$ we say that $\lambda$ is a partition of $n$, written as $\lambda \vdash n$. The number of strictly positive $\lambda_{i}$ (the parts of $\lambda$ ) is called the length of the partition $\lambda$ and denoted by $l(\lambda)$. We also use $l^{\circ}(\lambda)$ to denote the number of odd parts of $\lambda$. If $l^{\circ}(\lambda)=0$ we say that $\lambda$ is an even partition. The set of all partitions of length at most $n$ is denoted by $P_{+}(n)$, and typically we write $\lambda=\left(\lambda_{1}, \ldots, \lambda_{n}\right)$ for partitions in $P_{+}(n)$. The multiplicity of parts of size $i$ in the partition $\lambda$ is denoted by $m_{i}=m_{i}(\lambda)$. We sometimes use the multiplicities to write a partition $\lambda$ as $\left(1^{m_{1}} 2^{m_{2}} \ldots\right)$. When only a single multiplicity arises, i.e., a partition is of the form $\left(m^{n}\right)$, we refer to it as a rectangle. When $m_{i}(\lambda) \leqslant 1$ for all $i$ we say that $\lambda$ is a distinct partition. Given a partition $\lambda \in P_{+}(n)$ such that $\lambda_{1} \leqslant m$, we write $\left(m^{n}\right)-\lambda$ for the complement of $\lambda$ with respect to the rectangle $\left(m^{n}\right)$. That is, $\left(m^{n}\right)-\lambda:=\left(m-\lambda_{n}, \ldots, m-\lambda_{1}\right) \in P_{+}(n)$. The partition $\lambda^{\prime}=\left(\lambda_{1}^{\prime}, \lambda_{2}^{\prime}, \ldots\right)$ such that $\lambda_{i}^{\prime}=\sum_{j \geqslant i} m_{j}(\lambda)$ is called the conjugate of $\lambda$. Perhaps more simply, if we identify a partition $\lambda$ with its Young diagram (in which the parts are represented by $l(\lambda)$ left-aligned rows of boxes or squares, with $i$ th row containing $\lambda_{i}$ squares) then the parts $\lambda^{\prime}$ correspond to the columns of $\lambda$. Other special notation for partitions that we will employ is $2 \lambda:=\left(2 \lambda_{1}, 2 \lambda_{2}, \ldots\right)$ and $\lambda^{2}:=\left(\lambda_{1}, \lambda_{1}, \lambda_{2}, \lambda_{2}, \ldots\right)$, so that $2 \lambda$ (resp. $\left.\lambda^{2}\right)$ corresponds to the partition in which the length of each row (resp. column) of $\lambda$ has been doubled. The partition $\mu$ is contained in $\lambda$, denoted as $\mu \subset \lambda$, if $\mu_{i} \leqslant \lambda_{i}$ for all $i$, i.e., if the diagram of $\mu$ fits in the diagram of $\lambda$. We write $\mu \prec \lambda$ if $\mu \subset \lambda$ such that the interlacing condition $\lambda_{1} \geqslant \mu_{1} \geqslant \lambda_{2} \geqslant \mu_{2} \geqslant \cdots$ holds. (Alternatively, $\mu \prec \lambda$ if the skew shape $\lambda / \mu$ is a horizontal strip, see [26].) A partition $\lambda$ has empty 2 -core, written as 2 -core $(\lambda)=0$, if its diagram can be tiled by dominoes. For example, the partition $(5,4,4,1)$ has empty 2 -core since it admits the tiling

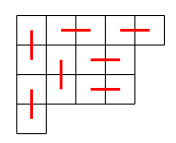

as well as four other such tilings.

Lemma 2.1. Let $\lambda \in P_{+}(n)$ and $m$ an even integer such that $m \geqslant n$. Then 2 -core $(\lambda)=0$ if and only if the ordered set

$$
\mathcal{A}_{\lambda}:=\left\{\lambda_{1}+m-1, \lambda_{2}+m-2, \ldots, \lambda_{n}+m-n, m-n-1, \ldots, 0\right\}
$$

contains $m$ even and $m$ odd integers.

Proof. The claim is (almost) trivially true by induction on the size of $\lambda$. Either we cannot remove a single domino from the border of $\lambda$, in which case $\lambda$ does not have a trivial 2-core (it is in fact a 2 -core itself) and is of the form $(n, n-1, \ldots, 1)$ for some positive $n$, or it is possible to remove a domino from the border of $\lambda$ to form a partition of size $|\lambda|-2$. The removal of a domino of shape $\square$ simply decreases one of the elements of $\mathcal{A}_{\lambda}$ by 2 , whereas the removal of a domino of shape $\boxminus$ decreases two consecutive elements of $\mathcal{A}_{\lambda}$ by 1 . The claim thus follows.

Given a square $s=(i, j) \in \lambda$ the arm-length, arm-colength, leg-length and leg-colength of $s$ are defined as

$$
\begin{array}{ll}
a(s)=a_{\lambda}(s):=\lambda_{i}-j, & a^{\prime}(s)=a_{\lambda}^{\prime}(s):=j-1, \\
l(s)=l_{\lambda}(s):=\lambda_{j}^{\prime}-i, & l^{\prime}(s)=l_{\lambda}^{\prime}(s):=i-1 .
\end{array}
$$


Extending this to type-C, we also set

$$
\hat{a}(s)=\hat{a}_{\lambda}(s):=\lambda_{i}+j-1, \quad \hat{l}(s)=\hat{l}_{\lambda}(s):=\lambda_{j}^{\prime}+i-1 .
$$

The rationale for denoting the set of partitions of length at most $n$ as $P_{+}(n)$ is that we identify such partitions with the dominant (integral) weights of $\operatorname{GL}(n, \mathbb{C})$. Frequently we also require the superset

$$
P(n):=\left\{\left(\lambda_{1}, \ldots, \lambda_{n}\right) \in \mathbb{Z}^{n}: \lambda_{1} \geqslant \lambda_{2} \geqslant \cdots \geqslant \lambda_{n}\right\}
$$

of all (integral) weights. By mild abuse of notation, we sometimes write for $\mu \in P(n+1)$ with $\mu_{n+1}=0$ that $\mu \in P_{+}(n)$, i.e., we consider $P_{+}(n)$ not just as a subset of $P(n)$ but also of $P(n+1)$.

For $\lambda$ a partition, define the statistic

$$
n(\lambda):=\sum_{s=(i, j) \in \lambda}(i-1)=\sum_{i \geqslant 1}(i-1) \lambda_{i}=\sum_{s=(i, j) \in \lambda}\left(\lambda_{j}^{\prime}-1\right) / 2=\sum_{i \geqslant 1}\left(\begin{array}{c}
\lambda_{i}^{\prime} \\
2
\end{array}\right) .
$$

This extends to skew shapes $\lambda / \mu$ in the obvious manner: $n(\lambda / \mu):=\sum_{i}(i-1)\left(\lambda_{i}-\mu_{i}\right)=$ $n(\lambda)-n(\mu)$. By further abuse of notation (since conjugation no longer makes sense) we will also use $n(\lambda)$ and $n\left(\lambda^{\prime}\right)$ for $\lambda \in P(n)$, defined as

$$
n(\lambda)=\sum_{i=1}^{n}(i-1) \lambda_{i} \quad \text { and } \quad n\left(\lambda^{\prime}\right)=\sum_{i=1}^{n}\left(\begin{array}{c}
\lambda_{i} \\
2
\end{array}\right) .
$$

Two describe some of our conjectures we also require three types of even and odd analogues of $n(\lambda)$ for $\lambda$ a partition:

$$
\begin{aligned}
n^{\mathrm{e} / \mathrm{o}}(\lambda) & :=\sum_{\begin{array}{c}
s=(i, j) \in \lambda \\
a(s)+l(s) \text { even/odd }
\end{array}}(i-1) \\
\hat{n}^{\mathrm{e} / \mathrm{o}}(\lambda):= & \sum_{\substack{s=(i, j) \in \lambda \\
a(s)+l(s) \text { even/odd }}}\left(\lambda_{j}^{\prime}-1\right) / 2
\end{aligned}
$$

and

$$
\bar{n}^{\mathrm{e} / \mathrm{o}}(\lambda):=\sum_{\substack{s=(i, j) \in \lambda \\ i+j \text { even/odd }}}(i-1) .
$$

Although

$$
n(\lambda)=n^{\mathrm{e}}(\lambda)+n^{\mathrm{o}}(\lambda)=\hat{n}^{\mathrm{e}}(\lambda)+\hat{n}^{\mathrm{o}}(\lambda)=\bar{n}^{\mathrm{e}}(\lambda)+\bar{n}^{\mathrm{o}}(\lambda),
$$

it is generally not true that $n^{\mathrm{e} / \mathrm{o}}(\lambda), \hat{n}^{\mathrm{e} / \mathrm{o}}(\lambda)$ and $\bar{n}^{\mathrm{e} / \mathrm{o}}(\lambda)$ (for fixed parity) coincide. In fact, $\hat{n}^{\mathrm{e} / \mathrm{o}}(\lambda)$ can take half-integer values.

We will in fact only use the above six functions for partitions that have empty 2-core. In that case, we have the following simple relation.

Lemma 2.2. For $\lambda$ a partition such that 2 -core $(\lambda)=0$,

$$
\bar{n}^{\mathrm{e} / \mathrm{o}}(\lambda)=2 \hat{n}^{\mathrm{o} / \mathrm{e}}(\lambda)-n^{\mathrm{e} / \mathrm{o}}(\lambda) .
$$


Proof. We again prove the claim by induction on the size of the partition $\lambda$. For $\lambda=0$ the claim is trivially true. Now let $\lambda$ be a partition of size at least two. Because $\lambda$ can be tiled by dominoes, it is always possible to remove a domino from its border to form a partition $\mu$ of size $|\lambda|-2$.

First assume it is possible to remove a domino of shape $\square$ such that the row-coordinate of the two boxes of the domino is $i$. Then

$$
n^{\mathrm{e} / \mathrm{o}}(\lambda)-n^{\mathrm{e} / \mathrm{o}}(\mu)=\bar{n}^{\mathrm{e} / \mathrm{o}}(\lambda)-\bar{n}^{\mathrm{e} / \mathrm{o}}(\mu)=2 \hat{n}^{\mathrm{e} / \mathrm{o}}(\lambda)-2 \hat{n}^{\mathrm{e} / \mathrm{o}}(\mu)=i-1 .
$$

The above gives

$$
n^{p_{1}}(\lambda)+\bar{n}^{p_{2}}(\lambda)-2 \hat{n}^{p_{3}}(\lambda)=n^{p_{1}}(\mu)+\bar{n}^{p_{2}}(\mu)-2 \hat{n}^{p_{3}}(\mu)
$$

irrespective of the choice of the parities $p_{1}, p_{2}$ and $p_{3}$. (This is consistent with the trivial fact that for $\lambda$ an even partition, $n^{p_{1}}(\lambda)=\bar{n}^{p_{2}}(\lambda)=\hat{n}^{p_{3}}(\lambda)$.)

Next assume that $\lambda^{\prime}$ is a distinct partition so that it is impossible to remove a domino of shape $\square$. We now only need to consider the first column of $\lambda$ from which a domino of shape $\square$ can be removed. If this is the $j$ th column, then $\lambda^{\prime}$ is a distinct partition such that $\lambda_{i}^{\prime}-\lambda_{i+1}^{\prime}=1$ for $1 \leqslant i \leqslant j-1$ and $\lambda_{j}^{\prime}-\lambda_{j+1}^{\prime} \geqslant 2$. (Of course, not each such a partition necessarily has an empty 2-core.) From a case-by-case analysis it follows that

$$
\begin{aligned}
& n^{\mathrm{e}}(\lambda)-n^{\mathrm{e}}(\mu)=\lambda_{1}^{\prime}-1, \quad n^{\mathrm{o}}(\lambda)-n^{\mathrm{o}}(\mu)=\lambda_{1}^{\prime}-2 j, \\
& \bar{n}^{\mathrm{e}}(\lambda)-\bar{n}^{\mathrm{e}}(\mu)= \begin{cases}\lambda_{1}^{\prime}-j & \text { if } \lambda_{1}^{\prime} \text { is odd, } \\
\lambda_{1}^{\prime}-j-1 & \text { if } \lambda_{1}^{\prime} \text { is even, }\end{cases} \\
& \bar{n}^{\mathrm{o}}(\lambda)-\bar{n}^{\mathrm{o}}(\mu)= \begin{cases}\lambda_{1}^{\prime}-j & \text { if } \lambda_{1}^{\prime} \text { is even, } \\
\lambda_{1}^{\prime}-j-1 & \text { if } \lambda_{1}^{\prime} \text { is odd, }\end{cases}
\end{aligned}
$$

and

$$
\begin{aligned}
& 2 \hat{n}^{\mathrm{e}}(\lambda)-2 \hat{n}^{\mathrm{e}}(\mu)= \begin{cases}2 \lambda_{1}^{\prime}-3 j & \text { if } \lambda_{1}^{\prime} \text { is even, } \\
2 \lambda_{1}^{\prime}-3 j-1 & \text { if } \lambda_{1}^{\prime} \text { is odd, }\end{cases} \\
& 2 \hat{n}^{\mathrm{o}}(\lambda)-2 \hat{n}^{\mathrm{o}}(\mu)= \begin{cases}2 \lambda_{1}^{\prime}-j-1 & \text { if } \lambda_{1}^{\prime} \text { is odd }, \\
2 \lambda_{1}^{\prime}-j-2 & \text { if } \lambda_{1}^{\prime} \text { is even. }\end{cases}
\end{aligned}
$$

Hence (2.2) again holds, but now with $p_{1}=p_{2} \neq p_{3}$.

\subsection{Generalised shifted factorials}

In this paper we require several types of shifted factorials. For complex $q$ such that $|q|<1$ the ordinary $q$-shifted factorial $(z ; q)_{\infty}$ is defined as

$$
(z ; q)_{\infty}:=\prod_{k \geqslant 1}\left(1-z q^{k-1}\right)
$$

This may be used to define $(z ; q)_{N}$ for arbitrary integer $N$ as

$$
(z ; q)_{N}:=\frac{(z ; q)_{\infty}}{\left(z q^{N} ; q\right)_{\infty}}
$$


In particular, if $N$ is nonnegative, $(z ; q)_{N}=\prod_{k=1}^{N}\left(1-z q^{k-1}\right)$ and if $N$ is a negative integer $1 /(q ; q)_{N}=0$. To generalise both definitions to the elliptic case, we need the elliptic gamma function $[46]$

$$
\Gamma_{p, q}(z):=\prod_{i, j=0}^{\infty} \frac{1-p^{i+1} q^{j+1} / z}{1-z p^{i} q^{j}},
$$

where $z \in \mathbb{C}^{*}$ and $p, q \in \mathbb{C}$ such that $|p|,|q|<1$. This function is symmetric in $p$ and $q$, satisfies the reflection formula $\Gamma_{p, q}(z) \Gamma_{p, q}(p q / z)=1$ and functional equation

$$
\Gamma_{p, q}(q z)=\theta(z ; p) \Gamma_{p, q}(z)
$$

where $\theta(z ; p)$ is the modified theta function

$$
\theta(z ; p):=(z ; p)_{\infty}(p / z ; p)_{\infty} .
$$

Since $\lim _{p \rightarrow 0} 1 / \Gamma_{p, q}(z)=(z ; q)_{\infty}$, the reciprocal of the elliptic gamma function can be viewed as an elliptic analogue of (2.3). The elliptic analogue of (2.4) is then

$$
(z ; q, p)_{N}:=\frac{\Gamma_{p, q}\left(z q^{N}\right)}{\Gamma_{p, q}(z)},
$$

which for nonnegative $N$ can also be expressed as

$$
(z ; q, p)_{N}=\prod_{k=1}^{N} \theta\left(z q^{k-1} ; p\right) .
$$

Clearly, $(z ; q, 0)_{N}=(z ; q)_{N}$.

Three important generalisations of $(z ; q, p)_{N}$ to the case of partitions are given by $[36,52]$

$$
\begin{aligned}
& C_{\lambda}^{0}(z ; q, t ; p):=\prod_{(i, j) \in \lambda} \theta\left(z q^{j-1} t^{1-i} ; p\right), \\
& C_{\lambda}^{-}(z ; q, t ; p):=\prod_{(i, j) \in \lambda} \theta\left(z q^{\lambda_{i}-j} t^{\lambda_{j}^{\prime}-i} ; p\right), \\
& C_{\lambda}^{+}(z ; q, t ; p):=\prod_{(i, j) \in \lambda} \theta\left(z q^{\lambda_{i}+j-1} t^{2-\lambda_{j}^{\prime}-i} ; p\right),
\end{aligned}
$$

where it is noted that

$$
C_{(N)}^{0}(z ; q, t ; p)=C_{(N)}^{-}(z ; q, t ; p)=(z ; q, p)_{N}
$$

and

$$
C_{(N)}^{+}(z ; q, t ; p)=\frac{(z ; q, p)_{2 N}}{(z ; q, p)_{N}}
$$

From the simple functional equations for the theta function

$$
\theta(p z ; p)=\theta(1 / z ; p)=-z^{-1} \theta(z ; p)
$$

it follows that the elliptic $C$-symbols satisfy the quasi-periodicities

$$
\begin{aligned}
& C_{\lambda}^{0}(p z ; q, t ; p)=(-z)^{-|\lambda|} q^{-n\left(\lambda^{\prime}\right)} t^{n(\lambda)} C_{\lambda}^{0}(z ; q, t ; p), \\
& C_{\lambda}^{-}(p z ; q, t ; p)=(-z)^{-|\lambda|} q^{-n\left(\lambda^{\prime}\right)} t^{-n(\lambda)} C_{\lambda}^{-}(z ; q, t ; p), \\
& C_{\lambda}^{+}(p z ; q, t ; p)=(-z q)^{-|\lambda|} q^{-3 n\left(\lambda^{\prime}\right)} t^{3 n(\lambda)} C_{\lambda}^{+}(z ; q, t ; p),
\end{aligned}
$$


as well as a long list of other simple identities, such as

$$
\begin{aligned}
& C_{\lambda^{\prime}}^{0}(z ; t, q ; p)=C_{\lambda}^{0}(p / z ; q, t ; p), \\
& C_{\lambda^{\prime}}^{-}(z ; t, q ; p)=C_{\lambda}^{-}(z ; q, t ; p), \\
& C_{\lambda^{\prime}}^{+}(z ; t, q ; p)=C_{\lambda}^{+}(p / z q t ; q, t ; p), \\
& C_{\lambda}^{0, \pm}(1 / z ; 1 / q, 1 / t ; p)=C_{\lambda}^{0, \pm}(p z ; q, t ; p), \\
& C_{2 \lambda}^{0, \pm}(z ; q, t ; p)=C_{\lambda}^{0, \pm}\left(z, z q ; q^{2}, t ; p\right), \\
& C_{\lambda^{2}}^{0}(z ; q, t ; p)=C_{\lambda}^{0}\left(z, z / t ; q, t^{2} ; p\right), \\
& C_{\lambda^{2}}^{-}(z ; q, t ; p)=C_{\lambda}^{-}\left(z, z t ; q, t^{2} ; p\right), \\
& C_{\lambda^{2}}^{+}(z ; q, t ; p)=C_{\lambda}^{+}\left(z / t, z / t^{2} ; q, t^{2} ; p\right), \\
& C_{\lambda}^{0, \pm}(z,-z ; q, t ; p)=C_{\lambda}^{0, \pm}\left(z^{2} ; q^{2}, t^{2} ; p^{2}\right),
\end{aligned}
$$

and

$$
\begin{aligned}
& C_{\lambda+\left(N^{n}\right)}^{0}(z ; q, t ; p)=C_{\left(N^{n}\right)}^{0}(z ; q, t ; p) C_{\lambda}^{0}\left(z q^{N} ; q, t ; p\right) \\
& C_{\lambda+\left(N^{n}\right)}^{-}(z ; q, t ; p)=C_{\left(N^{n}\right)}^{-}(z ; q, t ; p) C_{\lambda}^{-}(z ; q, t ; p) \frac{C_{\lambda}^{0}\left(z q^{N} t^{n-1} ; q, t ; p\right)}{C_{\lambda}^{0}\left(z t^{n-1} ; q, t ; p\right)} \\
& C_{\lambda+\left(N^{n}\right)}^{+}(z ; q, t ; p)=C_{\left(N^{n}\right)}^{+}(z ; q, t ; p) C_{\lambda}^{+}\left(z q^{2 N} ; q, t ; p\right) \frac{C_{\lambda}^{0}\left(z q^{2 N} t^{1-n} ; q, t ; p\right)}{C_{\lambda}^{0}\left(z q^{N} t^{1-n} ; q, t ; p\right)},
\end{aligned}
$$

where in the final set of identities it is assumed that $\lambda \in P_{+}(n)$ and $N$ is a nonnegative integer. Expressing $C_{\left(N^{n}\right)}^{0, \pm}(z ; q, t ; p)$ in terms of the elliptic shifted-factorial (2.6), we may use (2.13) to extend the elliptic $C$-symbols to arbitrary weights $\lambda \in P(n)$ :

$$
\begin{aligned}
& C_{\lambda}^{0}(z ; q, t ; p)=C_{\mu}^{0}\left(z q^{\lambda_{n}} ; q, t ; p\right) \prod_{i=1}^{n}\left(z t^{1-i} ; q, p\right)_{\lambda_{n}}, \\
& C_{\lambda}^{-}(z ; q, t ; p)=C_{\mu}^{-}(z ; q, t ; p) \frac{C_{\mu}^{0}\left(z q^{\lambda_{n}} t^{n-1} ; q, t ; p\right)}{C_{\mu}^{0}\left(z t^{n-1} ; q, t ; p\right)} \prod_{i=1}^{n}\left(z t^{n-i} ; q, p\right)_{\lambda_{n}}, \\
& C_{\lambda}^{+}(z ; q, t ; p)=C_{\mu}^{+}\left(z q^{2 \lambda_{n}} ; q, t ; p\right) \frac{C_{\mu}^{0}\left(z q^{2 \lambda_{n}} t^{1-n} ; q, t ; p\right)}{C_{\mu}^{0}\left(z q^{\lambda_{n}} t^{1-n} ; q, t ; p\right)} \prod_{i=1}^{n}\left(z q^{\lambda_{n}} t^{2-n-i} ; q, p\right)_{\lambda_{n}},
\end{aligned}
$$

where $\mu:=\left(\lambda_{1}-\lambda_{n}, \ldots, \lambda_{n-1}-\lambda_{n}, 0\right) \in P_{+}(n)$. All of the above identities, with the exception of (2.10) remain valid for non-dominant weights.

For all three elliptic $C$-symbols we also use their non-elliptic specialisations

$$
C_{\lambda}^{0, \pm}(z ; q, t):=C_{\lambda}^{0, \pm}(z ; q, t ; 0) .
$$

They satisfy the obvious analogues of (2.10)-(2.13), where it is noted that in the case of (2.10a), (2.10c) and (2.11) one first needs to eliminate an explicit $p$ in the argument on the right using (2.9) before setting $p$ to 0 . To avoid having to produce another five identities, we will always refer to the above relations - even in the non-elliptic case - when manipulating $C$-symbols. The reader should have no trouble writing down the explicit $p=0$ versions. For instance, in the case of (2.10a) on finds

$$
C_{\lambda^{\prime}}^{0}(z ; t, q)=(-z)^{|\lambda|} q^{-n\left(\lambda^{\prime}\right)} t^{n(\lambda)} C_{\lambda}^{0}(1 / z ; q, t),
$$

and so on. 
For all the shifted factorials as well as the elliptic gamma and modified theta functions adopt the usual multiplicative and plus-minus notations, such as

$$
C_{\lambda}^{0}\left(z_{1}, \ldots, z_{k} ; q, t ; p\right):=C_{\lambda}^{0}\left(z_{1} ; q, t ; p\right) \cdots C_{\lambda}^{0}\left(z_{k} ; q, t ; p\right)
$$

and

$$
\begin{aligned}
& C_{\lambda}^{0}\left(z^{ \pm} ; q, t ; p\right):=C_{\lambda}^{0}\left(z, z^{-1} ; q, t ; p\right), \\
& C_{\lambda}^{0}\left(w^{ \pm} z^{ \pm} ; q, t ; p\right):=C_{\lambda}^{0}\left(w z, w z^{-1}, w^{-1} z, w^{-1} z^{-1} ; q, t ; p\right) .
\end{aligned}
$$

To further shorten some of our expressions we also introduce the multiplicative well-poised ratio

$$
\Delta_{\lambda}^{0}\left(a \mid b_{1}, \ldots, b_{k} ; q, t ; p\right):=\frac{C_{\lambda}^{0}\left(b_{1}, \ldots, b_{k} ; q, t ; p\right)}{C_{\lambda}^{0}\left(a p q / b_{1}, \ldots, a p q / b_{k} ; q, t ; p\right)}
$$

and the non-multiplicative

$$
\Delta_{\lambda}\left(a \mid b_{1}, \ldots, b_{k} ; q, t ; p\right):=\frac{C_{2 \lambda^{2}}^{0}(a p q ; q, t ; p)}{C_{\lambda}^{-}(p q, t ; q, t ; p) C_{\lambda}^{+}(a, a p q / t ; q, t ; p)} \Delta_{\lambda}^{0}\left(a \mid b_{1}, \ldots, b_{k} ; q, t ; p\right) .
$$

Finally, there are six more non-elliptic $C$-symbols needed to describe some of our conjectures. They are defined as

$$
\begin{aligned}
& C_{\lambda}^{0, \mathrm{e} / \mathrm{o}}(z ; q, t):=\prod_{\substack{s=(i, j) \in \lambda \\
i+j \text { even/odd }}}\left(1-z q^{j-1} t^{1-i}\right), \\
& C_{\lambda}^{-, \mathrm{e} / \mathrm{o}}(z ; q, t):=\prod_{\substack{s=(i, j) \in \lambda \\
a(s)+l(s) \text { even/odd }}}\left(1-z q^{\lambda_{i}-j} t^{\lambda_{j}^{\prime}-i}\right), \\
& C_{\lambda}^{+, \mathrm{e} / \mathrm{o}}(z ; q, t):=\prod_{\substack{s=(i, j) \in \lambda \\
a(s)+l(s) \text { even/odd }}}\left(1-z q^{\lambda_{i}+j-1} t^{2-\lambda_{j}^{\prime}-i}\right) .
\end{aligned}
$$

Clearly, $C_{\lambda}^{\alpha}(z ; q, t)=C_{\lambda}^{\alpha, \mathrm{e}}(z ; q, t) C_{\lambda}^{\alpha, \mathrm{o}}(z ; q, t)$ for $\alpha \in\{0,+,-\}$.

Lemma 2.3. For $\lambda$ a partition,

$$
\begin{aligned}
& C_{\lambda^{\prime}}^{0, \mathrm{e} / \mathrm{o}}(z ; t, q)=(-z)^{\|\lambda\|^{\mathrm{e} / \mathrm{o}}} q^{-\bar{n}^{\mathrm{e} / \mathrm{o}}\left(\lambda^{\prime}\right)} t^{\bar{n}^{\mathrm{e} / \mathrm{o}}(\lambda)} C_{\lambda}^{0, \mathrm{e} / \mathrm{o}}(1 / z ; q, t), \\
& C_{\lambda^{\prime}}^{-, \mathrm{e} / \mathrm{o}}(z ; t, q)=C_{\lambda}^{-, \mathrm{e} / \mathrm{o}}(z ; q, t), \\
& C_{\lambda^{\prime}}^{+, \mathrm{e} / \mathrm{o}}(z ; t, q)=(-t z)^{|\lambda|^{\mathrm{e} / \mathrm{o}}} q^{-n^{\mathrm{e} / \mathrm{o}}\left(\lambda^{\prime}\right)-2 \hat{n}^{\mathrm{e} / \mathrm{o}}\left(\lambda^{\prime}\right)} t^{n^{\mathrm{e} / \mathrm{o}}(\lambda)+2 \hat{n}^{\mathrm{e} / \mathrm{o}}(\lambda)} C_{\lambda}^{+, \mathrm{e} / \mathrm{o}}(1 / q t z ; q, t),
\end{aligned}
$$

where $\|\lambda\|^{\mathrm{e} / \mathrm{o}}:=\mid\{(i, j) \in \lambda: i+j$ even/odd $\} \mid$ and $|\lambda|^{\mathrm{e} / \mathrm{o}}:=\mid\{s \in \lambda: a(s)+l(s)$ even/odd $\} \mid$.

We will again only be using the above for $\lambda$ a partition with empty 2-core, in which case we simply have $\|\lambda\|^{\mathrm{e} / \mathrm{o}}=|\lambda|^{\mathrm{e} / \mathrm{o}}=|\lambda| / 2$.

Proof. We will only show the last of the three identities. Applying definition (2.16c) to its left-hand side and interchanging $i$ and $j$ in the product leads to

$$
\begin{aligned}
C_{\lambda^{\prime}}^{+, \mathrm{e} / \mathrm{o}}(z ; t, q) & =\prod_{\substack{s=(i, j) \in \lambda \\
a(s)+l(s) \text { even/odd }}}\left(1-(q t z) q^{1-\lambda_{i}-j} t^{\lambda_{j}^{\prime}+i-2}\right) \\
& =C_{\lambda}^{+, \mathrm{e} / \mathrm{o}}(1 / q t z ; q, t) \prod_{\substack{s=(i, j) \in \lambda \\
a(s)+l(s) \text { even/odd }}}\left((-t z) q^{2-\lambda_{i}-j} t^{\lambda_{j}^{\prime}+i-2}\right) .
\end{aligned}
$$

By (2.1) the result now follows. 
In a similar manner it may be shown that (the $p=0$ case of) (2.11) dissects into even and odd cases as follows.

Lemma 2.4. For $\lambda$ a partition,

$$
\begin{aligned}
& C_{\lambda}^{0, \mathrm{e} / \mathrm{o}}(1 / z ; 1 / q, 1 / t)=(-z)^{-\|\lambda\|^{\mathrm{e} / \mathrm{o}}} q^{-\bar{n}^{\mathrm{e} / \mathrm{o}}\left(\lambda^{\prime}\right)} t^{\bar{n}^{\mathrm{e} / \mathrm{o}}(\lambda)} C_{\lambda}^{0, \mathrm{e} / \mathrm{o}}(z ; q, t), \\
& C_{\lambda}^{-, \mathrm{e} / \mathrm{o}}(1 / z ; 1 / q, 1 / t)=(-z)^{-|\lambda|^{\mathrm{e} / \mathrm{o}}} q^{n^{\mathrm{e} / \mathrm{o}}\left(\lambda^{\prime}\right)-2 \hat{n}^{\mathrm{e} / \mathrm{o}}\left(\lambda^{\prime}\right)} t^{n^{\mathrm{e} / \mathrm{o}}(\lambda)-2 \hat{n}^{\mathrm{e} / \mathrm{o}}(\lambda)} C_{\lambda}^{-, \mathrm{e} / \mathrm{o}}(z ; q, t), \\
& C_{\lambda}^{+, \mathrm{e} / \mathrm{o}}(1 / z ; 1 / q, 1 / t)=(-q z)^{-|\lambda|^{\mathrm{e} / \mathrm{o}}} q^{-n^{\mathrm{e} / \mathrm{o}}\left(\lambda^{\prime}\right)-2 \hat{n}^{\mathrm{e} / \mathrm{o}}\left(\lambda^{\prime}\right)} t^{n^{\mathrm{e} / \mathrm{o}}(\lambda)+2 \hat{n}^{\mathrm{e} / \mathrm{o}}(\lambda)} C_{\lambda}^{+, \mathrm{e} / \mathrm{o}}(z ; q, t) .
\end{aligned}
$$

From (2.10), (2.11) and Lemmas 2.3, 2.4 it follows that the rational functions defined in (1.9) and (1.13) satisfy the dualities

$$
\begin{aligned}
& c_{\lambda^{2}}(w, z ; q, t)=c_{\lambda^{2}}(1 / w, 1 / z ; 1 / q, 1 / t)=\left(\frac{q}{t}\right)^{|\lambda|} \frac{C_{\lambda^{2}}^{-}(t ; q, t)}{C_{\lambda^{2}}^{-}(q ; q, t)} d_{2 \lambda^{\prime}}(1 / w, 1 / z ; t, q), \\
& d_{2 \lambda}(w, z ; q, t)=d_{2 \lambda}(1 / w, 1 / z ; 1 / q, 1 / t)=\left(\frac{q}{t}\right)^{|\lambda|} \frac{C_{2 \lambda}^{-}(t ; q, t)}{C_{2 \lambda}^{-}(q ; q, t)} c_{\left(\lambda^{\prime}\right)^{2}}(1 / w, 1 / z ; t, q), \\
& e_{\lambda}(w, z ; q, t)=e_{\lambda}(1 / w, 1 / z ; 1 / q, 1 / t)=\left(-\frac{q}{t}\right)^{|\lambda|} \frac{C_{\lambda}^{-}\left(t^{2} ; q^{2}, t^{2}\right)}{C_{\lambda}^{-}\left(q^{2} ; q^{2}, t^{2}\right)} e_{\lambda^{\prime}}(1 / w, 1 / z ; t, q), \\
& f_{\lambda}(w, z ; q, t)=f_{\lambda}(1 / w, 1 / z ; 1 / q, 1 / t)=\left(\frac{q}{t}\right)^{|\lambda| / 2} \frac{C_{\lambda}^{-}(t ; q, t)}{C_{\lambda}^{-}(q ; q, t)} f_{\lambda^{\prime}}(1 / w, 1 / z ; t, q),
\end{aligned}
$$

where in the final line it is assumed that $\lambda$ is a partition with empty 2-core.

In Sections 5 and 7 we also use the $p, q$-symmetric versions of many of the generalised $q$-shifted factorials. For $\boldsymbol{\lambda}=\left(\lambda^{(1)}, \lambda^{(2)}\right)$ a pair of partitions or weights and $f_{\lambda}(q, t ; p)$ one of

$$
C_{\lambda}^{0, \pm}(\cdot ; q, t ; p), \quad \Delta_{\lambda}^{0}(\cdot \mid \cdot ; q, t ; p) \quad \text { or } \quad \Delta_{\lambda}(\cdot \mid \cdot ; q, t ; p),
$$

we set

$$
f_{\boldsymbol{\lambda}}(t ; p, q)=f_{\lambda^{(1)}}(p, t ; q) f_{\lambda^{(2)}}(q, t ; p) .
$$

Hence

$$
f_{\left(\lambda^{(1)}, \lambda^{(2)}\right)}(t ; p, q)=f_{\left(\lambda^{(2)}, \lambda^{(1)}\right)}(t ; q, p) .
$$

By slight abuse of notation we will also write $(n, m) \boldsymbol{\lambda}:=\left(n \lambda^{(1)}, m \lambda^{(2)}\right)$ for positive integers $n, m$, so that, for example,

$$
C_{(1,2) \boldsymbol{\lambda}}^{0}(a ; t ; p, q)=C_{\lambda^{(1)}}^{0}(a ; p, t ; q) C_{2 \lambda^{(2)}}^{0}(a ; q, t ; p) .
$$

Finally, $2 \boldsymbol{\lambda}:=(2,2) \boldsymbol{\lambda}$ and $\boldsymbol{\lambda}^{2}:=\left(\left(\lambda^{(1)}\right)^{2},\left(\lambda^{(2)}\right)^{2}\right)$.

\section{$2.3 \quad$ Elliptic hypergeometric series}

Our proof of Theorem 1.2 relies on (the $p \rightarrow 0$ limit of) two higher-dimensional quadratic summation formulas for elliptic hypergeometric series. In the one-dimensional case the simplest form an elliptic hypergeometric series can take is $[13,50,52]$

$$
\sum_{k=0}^{\infty} \Delta_{(k)}\left(a \mid t, b_{1}, \ldots, b_{r} ; q, t ; p\right)=\sum_{k=0}^{\infty} \frac{(a p q ; q, p)_{2 k}}{(a ; q, p)_{2 k}} \frac{\left(a, b_{1}, \ldots, b_{r} ; q, p\right)_{k}}{\left(p q, c_{1}, \ldots, c_{r} ; q, p\right)_{k}}
$$


where, for reasons of convergence, it is assumed that one of the $b_{i}$ is of the form $q^{-N}$ with $N$ a nonnegative integer so that the series terminates. If the upper and lower parameters satisfy $a b_{1} \cdots b_{r}(p q)^{3}=c_{1} \cdots c_{r}$ the series (2.17) is said to be balanced, and if $b_{i} c_{i}=a p q$ for all $i$ it is said to be very-well poised. If both these conditions are satisfied then (2.17) is an elliptic function (in multiplicative form) in each of the variables $a, b_{1}, \ldots, b_{r}$, see, e.g., [50].

The most important identity for one-dimensional elliptic hypergeometric series corresponds to (2.17) for $r=5$, and is given by Frenkel and Turaev's elliptic analogue of Jackson's sum [12]:

$$
\begin{gathered}
\sum_{k=0}^{N} \frac{(a p q ; q, p)_{2 k}}{(a ; q, p)_{2 k}} \frac{\left(a, b, c, d, e, q^{-N} ; q, p\right)_{k}}{\left(p q, a p q / b, a p q / c, a p q / d, a p q / e, a p q^{N+1} ; q, p\right)_{k}} \\
=\frac{(a p q, a p q / b c, a p q / b d, a p q / c d ; q, p)_{N}}{(a p q / b, a p q / c, a p q / d, a p q / b c d ; q, p)_{N}}
\end{gathered}
$$

where $b c d e q^{-N}=a^{2} p q$. Four other balanced, very-well poised instances of (2.17) for $r=7$ that admit closed-form evaluations are given by

$$
\begin{gathered}
\sum_{k=0}^{N} \frac{(a p q ; q, p)_{2 k}}{(a ; q, p)_{2 k}} \frac{\left(a, b q, a b q^{N}, q^{-N} ; q, p\right)_{k}}{\left(p q, a p / b, p q^{1-N} / b, a p q^{N+1} ; q, p\right)_{k}} \frac{\left(a p / b ; q, p^{2}\right)_{2 k}}{\left(a b p q ; q, p^{2}\right)_{2 k}} \\
=\frac{\theta\left(a b p ; p^{2}\right)}{\theta\left(a b p q^{2 N} ; p^{2}\right)} \frac{(a p q ; q, p)_{N}}{(b ; q, p)_{N}} \frac{\left(p q, b^{2} q ; q, p^{2}\right)_{N}}{\left(a b p, a p^{2} / b ; q, p^{2}\right)_{N}} \\
\sum_{k=0}^{N} \frac{(a p q ; q, p)_{2 k}}{(a ; q, p)_{2 k}} \frac{\left(a, b p, a b q^{N}, q^{-N} ; q, p\right)_{k}}{\left(p q, a q / b, p q^{1-N} / b, a p q^{N+1} ; q, p\right)_{k}} \frac{\left(a q / b ; q^{2}, p\right)_{k}}{\left(a b p q ; q^{2}, p\right)_{k}} \\
=\chi\left(N \text { even)} \frac{\left(q, b^{2} ; q^{2}, p\right)_{N / 2}}{\left(a b p q, a p q^{2} / b ; q^{2}, p\right)_{N / 2}} \frac{(a p q ; q, p)_{N}}{(b ; q, p)_{N}},\right. \\
\sum_{k=0}^{N} \frac{(a p q ; q, p)_{2 k}}{(a ; q, p)_{2 k}} \frac{\left(b, p q / b, q^{N+1}, q^{-N} ; q, p\right)_{k}}{\left(a p q / b, a b, a p q^{-N}, a p q^{N+1} ; q, p\right)_{k}} \frac{\left(a^{2} ; q^{2}, p\right)_{k}}{\left(p q^{2} ; q^{2}, p\right)_{k}} \\
=\frac{(a p q, b / a ; q, p)_{N}}{(q / a, a b p ; q, p)_{N}} \frac{\left(a b p q^{-N} ; q^{2}, p\right)_{N}}{\left(b q^{-N} / a ; q^{2}, p\right)_{N}}
\end{gathered}
$$

and

$$
\begin{gathered}
\sum_{k=0}^{N} \frac{\left(a^{2} p^{2} q^{2} ; q^{2}, p^{2}\right)_{2 k}}{\left(a^{2} ; q^{2}, p^{2}\right)_{2 k}} \frac{\left(a^{2}, b^{2} p^{2} q^{2}, a^{2} b^{2} q^{2 N}, q^{-2 N} ; q^{2}, p^{2}\right)_{k}}{\left(p^{2} q^{2}, a^{2} / b^{2}, p^{2} q^{2-2 N} / b^{2}, a^{2} p^{2} q^{2 N+2} ; q^{2}, p^{2}\right)_{k}} \frac{(a / b ; q, p)_{2 k}}{(a b p q ; q, p)_{2 k}} \\
\quad=\frac{(a b ; q, p)_{2 N}}{(a b p q ; q, p)_{2 N}} \frac{\left(-p q, b^{2} p q ; q, p\right)_{N}}{(a b,-a / b ; q, p)_{N}} \frac{\left(a^{2} p^{2} q^{2} ; q^{2}, p^{2}\right)_{N}}{\left(b^{2} ; q^{2}, p^{2}\right)_{N}}
\end{gathered}
$$

The last three identities are equations (1.10), (1.15) and (1.4) of [54] respectively. The identity (2.19a) does not appear to have been stated before. It follows by inverting, using [53, Lemma 3.1], the elliptic Jackson sum (2.18) with

$$
(b, c, d, e, p) \mapsto\left(b^{2} q, a q^{N} / b, a p q^{N} / b, p q^{-N}, p^{2}\right) .
$$

Because of the occurrence of base $q$ and $q^{2}$ (and nomes $p$ and $p^{2}$ ), the above identities are commonly referred to as quadratic summation formulas. In Section 7 we obtain higherdimensional analogues of $(2.19 \mathrm{a}),(2.19 \mathrm{~b})$ and $(2.19 \mathrm{~d})$. Two of these play a key role in our proof of Theorem 1.2. We remark that higher-dimensional analogues of a different type of quadratic elliptic hypergeometric series, in which the term $(a p q ; q, p)_{2 k} /(a ; q, p)_{2 k}$ in $(2.17)$ is replaced by $(a p q ; q, p)_{3 k} /(a ; q, p)_{3 k}$, were recently considered in [44]. 


\section{Schur functions and classical branching rules}

In this section we briefly review the definitions of the Schur functions of classical type as well as their occurrence in some of the branching rules stated in Section 1. For a more in-depth treatment we refer the reader to [19, 21, 25, 26, 31, 42].

Let $\Lambda_{n}:=\mathbb{Z}\left[x_{1}, \ldots, x_{n}\right]^{S_{n}}$ denote the ring of symmetric functions in $n$ variables, and $\Lambda$ the ring of symmetric functions in countably many variables, see $[26,51]$. The monomial symmetric functions $\left\{m_{\lambda}\right\}_{\lambda \in P_{+}(n)}$ and $\left\{m_{\lambda}\right\}_{\lambda}$, where

$$
m_{\lambda}\left(x_{1}, \ldots, x_{n}\right)=\sum_{w \in S_{n} / S_{n}^{\lambda}} w\left(x^{\lambda}\right), \quad \lambda \in P_{+}(n),
$$

form $\mathbb{Z}$-bases of $\Lambda_{n}$ and $\Lambda$ respectively. The elementary, complete and power-sum symmetric functions $e_{r}, h_{r}$ and $p_{r}$ are defined in terms of the monomial symmetric functions as

$$
\begin{aligned}
e_{r} & :=m_{\left(1^{r}\right)}=\sum_{1<i_{1}<i_{2}<\cdots<i_{r}} x_{i_{1}} x_{i_{2}} \cdots x_{i_{r}}, \\
h_{r} & :=\sum_{\lambda \vdash r} m_{\lambda}=\sum_{1 \leqslant i_{1} \leqslant i_{2} \leqslant \cdots \leqslant i_{r}} x_{i_{1}} x_{i_{2}} \cdots x_{i_{r}}, \\
p_{r} & :=m_{(r)}=\sum_{i \geqslant 1} x_{i}^{r} .
\end{aligned}
$$

These functions form algebraic bases of either $\Lambda$ (in the case of the $e_{r}$ and $h_{r}$ ) or of $\Lambda_{\mathbb{Q}}:=\Lambda \otimes_{\mathbb{Z}} \mathbb{Q}$ (in the case of the power sums).

A number of classical branching rules for universal characters discussed below are related by the involution $\omega$ on $\Lambda$ defined by $\omega\left(h_{r}\right)=e_{r}$ or $\omega\left(p_{r}\right)=(-1)^{r-1} p_{r}$ for all $r \geqslant 1$.

The ordinary (or GL $(n)$ ) Schur function indexed by the partition $\lambda$ is defined as

$$
s_{\lambda}\left(x_{1}, \ldots, x_{n}\right):=\frac{\operatorname{det}_{1 \leqslant i, j \leqslant n}\left(x_{i}^{\lambda_{j}+n-j}\right)}{\prod_{1 \leqslant i<j \leqslant n}\left(x_{i}-x_{j}\right)}
$$

if $l(\lambda) \leqslant n$ and 0 otherwise. To simultaneously extend this to $\Lambda$ as well as skew shapes, we use the Jacobi-Trudi identity or its dual [26, pp. 70-71]:

$$
s_{\lambda / \mu}:=\operatorname{det}_{1 \leqslant i, j \leqslant n}\left(h_{\lambda_{i}-\mu_{j}-i+j}\right)=\operatorname{det}_{1 \leqslant i, j \leqslant m}\left(e_{\lambda_{i}^{\prime}-\mu_{j}^{\prime}-i+j}\right),
$$

where $n$ and $m$ are arbitrary integers such that $n \geqslant l(\lambda)$ and $m \geqslant \lambda_{1}$. Obviously, $\omega\left(s_{\lambda / \mu}\right)=$ $s_{\lambda^{\prime} / \mu^{\prime}}$. The Littlewood-Richardson coefficients $c_{\mu \nu}^{\lambda}$ may now be defined by

$$
s_{\lambda / \mu}=\sum_{\nu} c_{\mu \nu}^{\lambda} s_{\nu}
$$

From (3.2) it follows that $s_{\lambda / \mu}=s_{\left(\left(m^{n}\right)-\mu\right) /\left(\left(m^{n}\right)-\lambda\right)}$ for $\lambda \subset\left(m^{n}\right)$ so that the LittlewoodRichardson coefficients satisfy the complementation symmetry

$$
c_{\mu \nu}^{\lambda}=c_{\left(m^{n}\right)-\lambda, \nu}^{\left(m^{n}\right)-\mu} \quad \text { for } \lambda \subset\left(m^{n}\right) .
$$

For $\lambda$ a partition, the universal orthogonal and symplectic characters indexed by $\lambda$ are given by [19, Definition 2.1.1]

$$
\begin{aligned}
& \mathrm{o}_{\lambda}:=\operatorname{det}_{1 \leqslant i, j \leqslant n}\left(h_{\lambda_{i}-i+j}-h_{\lambda_{i}-i-j}\right) \\
& \mathrm{sp}_{\lambda}:=\operatorname{det}_{1 \leqslant i, j \leqslant m}\left(e_{\lambda_{i}^{\prime}-i+j}-e_{\lambda_{i}^{\prime}-i-j}\right),
\end{aligned}
$$


with $n$ and $m$ as above. Hence $\omega\left(\mathrm{o}_{\lambda}\right)=\mathrm{sp}_{\lambda^{\prime}}$. In particular,

$$
g_{\lambda}\left(x_{1}^{ \pm}, \ldots, x_{n}^{ \pm}, 0, \ldots\right)= \begin{cases}g_{2 n, \lambda}\left(x_{1}, x_{2}, \ldots, x_{n}\right) & \text { if } \lambda \in P_{+}(n), \\ 0 & \text { otherwise }\end{cases}
$$

where $g=\operatorname{sp}($ resp. $g=0$ ) corresponds to an orthogonal or symplectic character indexed by $\lambda$. We add to the above the universal special orthogonal character indexed by $\lambda$ as

$$
\operatorname{so}_{\lambda}:=\operatorname{det}_{1 \leqslant i, j \leqslant n}\left(h_{\lambda_{i}-i+j}+h_{\lambda_{i}-i-j+1}\right)=\operatorname{det}_{1 \leqslant i, j \leqslant m}\left(e_{\lambda_{i}^{\prime}-i+j}+e_{\lambda_{i}^{\prime}-i-j+1}\right),
$$

so that $\omega\left(\mathrm{so}_{\lambda}\right)=\mathrm{so}_{\lambda^{\prime}}$. The character $\mathrm{so}_{\lambda}$ is the unique symmetric function such that

$$
\operatorname{so}_{\lambda}\left(x_{1}^{ \pm}, \ldots, x_{n}^{ \pm}, 0, \ldots\right)= \begin{cases}\operatorname{so}_{2 n+1, \lambda}\left(x_{1}, x_{2}, \ldots, x_{n}\right) & \text { if } \lambda \in P_{+}(n) \\ 0 & \text { otherwise }\end{cases}
$$

where $\mathrm{so}_{2 n+1, \lambda}$ is the odd-orthogonal Schur function indexed by $\lambda$. The character so $_{\lambda}$ may readily be related to the universal symplectic and orthogonal characters as

$$
\operatorname{so}_{\lambda}=\sum_{\mu^{\prime} \prec \lambda^{\prime}} \operatorname{sp}_{\mu}=\sum_{\mu \prec \lambda} \mathrm{o}_{\mu}
$$

and

$$
\operatorname{sp}_{\lambda}=\sum_{\mu \prec \lambda}(-1)^{|\lambda / \mu|} \mathrm{so}_{\mu}, \quad \mathrm{o}_{\lambda}=\sum_{\mu^{\prime} \prec \lambda^{\prime}}(-1)^{|\lambda / \mu|} \mathrm{so}_{\mu} .
$$

For the actual symplectic, orthogonal and odd-orthogonal Schur functions we have [25]

$$
\begin{aligned}
& \operatorname{sp}_{2 n, \lambda}\left(x_{1}, \ldots, x_{n}\right)=\frac{\operatorname{det}_{1 \leqslant i, j \leqslant n}\left(x_{i}^{\lambda_{j}+2 n-j+1}-x_{i}^{-\lambda_{j}+j-1}\right)}{\prod_{i=1}^{n}\left(x_{i}^{2}-1\right) \prod_{1 \leqslant i<j \leqslant n}\left(x_{i}-x_{j}\right)\left(x_{i} x_{j}-1\right)}, \\
& \mathrm{o}_{2 n, \lambda}\left(x_{1}, \ldots, x_{n}\right)=f_{l(\lambda), n} \frac{\operatorname{det}_{1 \leqslant i, j \leqslant n}\left(x_{i}^{\lambda_{j}+2 n-j-1}+x_{i}^{-\lambda_{j}+j-1}\right)}{\prod_{1 \leqslant i<j \leqslant n}\left(x_{i}-x_{j}\right)\left(x_{i} x_{j}-1\right)},
\end{aligned}
$$

where $f_{n, n}=1$ and $f_{i, n}=1 / 2$ if $i<n$, and

$$
\operatorname{so}_{2 n+1, \lambda}\left(x_{1}, \ldots, x_{n}\right)=\frac{\operatorname{det}_{1 \leqslant i, j \leqslant n}\left(x_{i}^{\lambda_{j}+2 n-j}-x_{i}^{-\lambda_{j}+j-1}\right)}{\prod_{i=1}^{n}\left(x_{i}-1\right) \prod_{1 \leqslant i<j \leqslant n}\left(x_{i}-x_{j}\right)\left(x_{i} x_{j}-1\right)} .
$$

Littlewood [25] and Koike and Terada [19] proved some very general branching formulas for the classical groups. For example, in the universal case [19, Theorem 2.3.1],

$$
s_{\lambda}=\sum_{\mu}\left(\sum_{\nu \text { even }} c_{\mu \nu}^{\lambda}\right) \mathrm{o}_{\mu}=\sum_{\mu}\left(\sum_{\nu^{\prime} \text { even }} c_{\mu \nu}^{\lambda}\right) \operatorname{sp}_{\mu} .
$$

By (3.3) it is not hard to show that (1.4) and (1.10) follow from (3.11). Indeed,

$$
s_{\left(m^{r-1}, m-p\right)}=\sum_{\mu} \sum_{\nu \text { even }} c_{\mu \nu}^{\left(m^{r-1}, m-p\right)} \mathrm{o}_{\mu}=\sum_{\mu \subset\left(m^{r}\right)} \sum_{\nu \text { even }} c_{\left(1^{p}\right), \nu}^{\mu} \mathrm{o}_{\left(m^{r}\right)-\mu} .
$$

By the $e$-Pieri rule [26, p. 73],

$$
c_{\left(1^{p}\right), \nu}^{\mu}= \begin{cases}1 & \text { if } \nu^{\prime} \prec \mu^{\prime} \text { and }|\mu / \nu|=p, \\ 0 & \text { otherwise. }\end{cases}
$$


Hence

$$
s_{\left(m^{r-1}, m-p\right)}=\sum_{\mu \subset\left(m^{r}\right)} \sum_{\substack{\nu^{\prime} \prec \mu^{\prime} \\ \nu \text { even } \\ \nu|=| \mu \mid-p}} \mathrm{O}_{\left(m^{r}\right)-\mu} \cdot
$$

Since

$$
\sum_{\substack{\nu^{\prime} \prec \mu^{\prime} \\ \nu \text { even } \\|\nu|=|\mu|-p}} 1= \begin{cases}1 & \text { if } l^{\circ}(\mu)=p \\ 0 & \text { otherwise }\end{cases}
$$

the branching rule (1.4) follows.

We also remark that the three universal branching rules (1.4), (1.10) and (1.11) are not independent. Obviously, (1.10) follows from (1.4) by application of $\omega$ and vice versa. Also, the rectangular (i.e., $p=0$ ) cases of each of the branching rules are related via (3.9). For example, from (1.11) and (3.9a), and the fact that $\left(m^{r}\right)-\lambda \prec\left(m^{r}\right)-\mu$ (for $\lambda, \mu \subset\left(m^{r}\right)$ ) implies $\mu \succ \lambda$, it follows that

$$
\begin{aligned}
s_{\left(m^{r}\right)} & =\sum_{\lambda \subset\left(m^{r}\right)}(-1)^{|\lambda|} \mathrm{SO}_{\left(m^{r}\right)-\lambda}=\sum_{\lambda \subset\left(m^{r}\right)}(-1)^{|\lambda|} \sum_{\mu \succ \lambda} \mathrm{O}_{\left(m^{r}\right)-\mu} \\
& =\sum_{\mu \subset\left(m^{r}\right)} \sum_{\lambda \prec \mu}(-1)^{|\lambda|} \mathrm{O}_{\left(m^{r}\right)-\mu}=\sum_{\substack{\mu \subset\left(m^{r}\right) \\
\mu \text { even }}} \mathrm{O}_{\left(m^{r}\right)-\mu},
\end{aligned}
$$

where in the last step we have used

$$
\sum_{\lambda \prec \mu}(-1)^{|\lambda|}= \begin{cases}1 & \text { if } \mu \text { is even, } \\ 0 & \text { otherwise. }\end{cases}
$$

Conversely, from (1.10) and (3.9b),

$$
\begin{aligned}
s_{\left(m^{r}\right)} & =\sum_{\substack{\lambda \subset\left(m^{r}\right) \\
\lambda \text { even }}} \mathrm{O}_{\left(m^{r}\right)-\lambda}=\sum_{\substack{\lambda \subset\left(m^{r}\right) \\
\lambda \text { even }}} \sum_{\mu^{\prime} \succ \lambda^{\prime}}(-1)^{|\mu / \lambda|} \mathrm{SO}_{\left(m^{r}\right)-\mu} \\
& =\sum_{\mu \subset\left(m^{r}\right)}(-1)^{|\mu|} \sum_{\substack{\lambda^{\prime} \prec \mu^{\prime} \\
\lambda \text { even }}} \operatorname{so}_{\left(m^{r}\right)-\mu}=\sum_{\mu \subset\left(m^{r}\right)}(-1)^{|\mu|} \mathrm{SO}_{\left(m^{r}\right)-\mu},
\end{aligned}
$$

since there is a unique even partition $\lambda$ such that $\lambda^{\prime} \prec \mu^{\prime}$.

In the $q, t$-case, we neither have analogues of (3.9) nor of (3.11), making the proof of Theorem 1.2 much harder than in the classical case. As we shall see, however, (1.8a) and (1.8b) are related by the $q, t$-analogue of the involution $\omega$.

\section{Macdonald-Koornwinder theory}

In this section we survey some necessary background material from the theory of Macdonald and Koornwinder polynomials, covering Macdonald polynomials, $\mathrm{BC}_{n}$-symmetric (Macdonald) interpolation polynomials, (lifted) Koornwinder polynomials and elliptic interpolation functions. 


\subsection{Macdonald polynomials}

Let $0<|q|,|t|<1$. For $f$ an $S_{n}$-symmetric function (not necessarily an $S_{n}$-symmetric Laurent polynomial) we define

$$
\langle f\rangle_{q, t}^{(n)}:=\frac{1}{S_{n}(q, t)} \frac{1}{n !(2 \pi \mathrm{i})^{n}} \int_{\mathbb{T}^{n}} f(z) \prod_{1 \leqslant i<j \leqslant n} \frac{\left(z_{i} / z_{j}, z_{j} / z_{i} ; q\right)_{\infty}}{\left(t z_{i} / z_{j}, t z_{j} / z_{i} ; q\right)_{\infty}} \frac{\mathrm{d} z_{1}}{z_{1}} \cdots \frac{\mathrm{d} z_{n}}{z_{n}},
$$

where $\mathbb{T}$ is the positively-oriented unit circle and [26]

$$
S_{n}(q, t):=\frac{1}{n !(2 \pi \mathrm{i})^{n}} \int_{\mathbb{T}^{n}} \prod_{1 \leqslant i<j \leqslant n} \frac{\left(z_{i} / z_{j}, z_{j} / z_{i} ; q\right)_{\infty}}{\left(t z_{i} / z_{j}, t z_{j} / z_{i} ; q\right)_{\infty}} \frac{\mathrm{d} z_{1}}{z_{1}} \cdots \frac{\mathrm{d} z_{n}}{z_{n}}=\prod_{i=1}^{n} \frac{\left(t, q t^{i-1} ; q\right)_{\infty}}{\left(q, t^{i} ; q\right)_{\infty}} .
$$

Recall the dominance (partial) order on partitions: for $\lambda, \mu \vdash m, \lambda \geqslant \mu$ if $\lambda_{1}+\cdots+\lambda_{i} \geqslant$ $\mu_{1}+\cdots+\mu_{i}$ for all $i \geqslant 1$, and $\lambda>\mu$ if $\lambda \geqslant \mu$ and $\lambda \neq \mu$. Also let $x^{-1}:=\left(x_{1}^{-1}, \ldots, x_{n}^{-1}\right)$. Then Macdonald polynomials $P_{\lambda}=P_{\lambda}(q, t)=P_{\lambda}(x ; q, t)$ for $\lambda \in P_{+}(n)$ are the unique homogeneous symmetric functions in $\Lambda_{\mathbb{Q}(q, t)}:=\Lambda \otimes_{\mathbb{Z}} \mathbb{Q}(q, t)=\mathbb{Q}(q, t)\left[x_{1}, \ldots, x_{n}\right]^{S_{n}}$ of the form

$$
P_{\lambda}=m_{\lambda}+\sum_{\mu<\lambda} c_{\lambda \mu}(q, t) m_{\mu}
$$

such that (for $0<|q|,|t|<1$ )

$$
\left\langle P_{\lambda}(x ; q, t) P_{\mu}\left(x^{-1} ; q, t\right)\right\rangle_{q, t}^{(n)}=0
$$

if $\lambda \neq \mu$, see [26, pp. 368-376]. For $\lambda=\mu$,

$$
\begin{aligned}
\left\langle P_{\lambda}(x ; q, t) P_{\lambda}\left(x^{-1} ; q, t\right)\right\rangle_{q, t}^{(n)} & =\prod_{1 \leqslant i<j \leqslant n} \frac{\left(t^{j-i+1}, q t^{j-i-1} ; q\right)_{\lambda_{i}-\lambda_{j}}}{\left(t^{j-i}, q t^{j-i} ; q\right)_{\lambda_{i}-\lambda_{j}}} \\
& =\frac{C_{\lambda}^{0}\left(t^{n} ; q, t\right) C_{\lambda}^{-}(q ; q, t)}{C_{\lambda}^{0}\left(q t^{n-1} ; q, t\right) C_{\lambda}^{-}(t ; q, t)} .
\end{aligned}
$$

The Macdonald polynomials satisfy the symmetry

$$
P_{\lambda}(x ; q, t)=P_{\lambda}(x ; 1 / q, 1 / t)
$$

and (dual) Cauchy identity

$$
\sum_{\lambda \subset\left(m^{n}\right)} P_{\lambda}\left(x_{1}, \ldots, x_{n} ; q, t\right) P_{\lambda^{\prime}}\left(y_{1}, \ldots, y_{m} ; t, q\right)=\prod_{i=1}^{n} \prod_{j=1}^{m}\left(1+x_{i} y_{j}\right) .
$$

Since

$$
P_{\left(\lambda_{1}+1, \ldots, \lambda_{n}+1\right)}(x ; q, t)=\left(x_{1} \cdots x_{n}\right) P_{\left(\lambda_{1}, \ldots, \lambda_{n}\right)}(x ; q, t),
$$

they can be extended from $\lambda \in P_{+}(n)$ to arbitrary weights $\lambda \in P(n)$ via

$$
P_{\lambda}(x ; q, t)=\left(x_{1} \cdots x_{n}\right)^{\lambda_{n}} P_{\mu}(x ; q, t),
$$

where $\mu:=\left(\lambda_{1}-\lambda_{n}, \ldots, \lambda_{n-1}-\lambda_{n}, 0\right) \in P_{+}(n)$. Then $\left\{P_{\lambda}(q, t)\right\}_{\lambda \in P(n)}$ forms a $\mathbb{Q}(q, t)$-basis of the ring of $S_{n}$-symmetric Laurent polynomials, which in the following we will denote by $\Lambda_{\mathrm{GL}(n)}$. Since,

$$
P_{\lambda}(x ; q, t) P_{\mu}\left(x^{-1} ; q, t\right)=P_{\left(\lambda_{1}-m, \ldots, \lambda_{n}-m\right)}(x ; q, t) P_{\left(\mu_{1}-m, \ldots, \mu_{n}-m\right)}\left(x^{-1} ; q, t\right),
$$


where $m=\min \left\{\lambda_{n}, \mu_{n}\right\}$, the orthogonality (4.3) and evaluation (4.4) extend to all $\lambda, \mu \in P(n)$. In the case of (4.4) for $\lambda$ a non-dominant weight, the first expression on the right should be used as both $C_{\lambda}^{-}(z q ; q, t)$ and $1 / C_{\lambda}^{0}\left(z q t^{n-1} ; q, t\right)$ have a pole (of order one) at $z=1$.

For later use we note that by (4.7) and the complementation symmetry for Macdonald polynomials (see, e.g., [3])

$$
P_{\left(\lambda_{1}, \ldots, \lambda_{n}\right)}\left(x^{-1} ; q, t\right)=\left(x_{1} \cdots x_{n}\right)^{-\lambda_{1}} P_{\left(\lambda_{1}-\lambda_{n}, \ldots, \lambda_{1}-\lambda_{2}, 0\right)}(x ; q, t)
$$

we have the further symmetry

$$
P_{\left(\lambda_{1}, \ldots, \lambda_{n}\right)}\left(x^{-1} ; q, t\right)=P_{\left(-\lambda_{n}, \ldots,-\lambda_{1}\right)}(x ; q, t) .
$$

If the involution $\omega$ on $\Lambda$ is extended to the following automorphism of $\Lambda_{\mathbb{Q}(q, t)}$ :

$$
\omega_{q, t}\left(p_{r}\right)=(-1)^{r-1} \frac{1-q^{r}}{1-t^{r}} p_{r}
$$

for all $r \geqslant 1$, then

$$
\omega_{q, t}\left(P_{\lambda}(q, t)\right)=Q_{\lambda^{\prime}}(t, q),
$$

where

$$
Q_{\lambda}(q, t):=\frac{C_{\lambda}^{-}(t ; q, t)}{C_{\lambda}^{-}(q ; q, t)} P_{\lambda}(q, t)
$$

\section{2 $\quad \mathrm{BC}_{n}$-symmetric interpolation polynomials}

Let $R$ be a coefficient ring or field, such as $\mathbb{Q}(q, t)\left[s^{ \pm}\right]$or $\mathbb{Q}\left(q, t, t_{0}, t_{1}, t_{2}, t_{3}\right)$. A polynomial $f \in R\left[x_{1}^{ \pm}, \ldots, x_{n}^{ \pm}\right]$is said to be $\mathrm{BC}_{n}$-symmetric if it is symmetric under the canonical action of the hyperoctahedral group $W:=S_{n} \ltimes(\mathbb{Z} / 2 \mathbb{Z})^{n}$ on $R\left[x_{1}^{ \pm}, \ldots, x_{n}^{ \pm}\right]$. Let $x:=\left(x_{1}, \ldots, x_{n}\right)$. The monomial basis in the ring of $\mathrm{BC}_{n}$-symmetric polynomials, $\Lambda_{\mathrm{BC}(n)}=R\left[x_{1}^{ \pm}, \ldots, x_{n}^{ \pm}\right]^{W}$, is given by $\left\{m_{\lambda}^{W}\right\}_{\lambda \in P_{+}(n)}$, where

$$
m_{\lambda}^{W}=m_{\lambda}^{W}(x):=\sum_{w \in W / W^{\lambda}} w\left(x^{\lambda}\right) .
$$

Any non-constant $\mathrm{BC}_{n}$-symmetric polynomial is necessarily inhomogeneous. It will thus be convenient to extend the dominance order from partitions of the same size to all partitions in the obvious way: $\lambda \geqslant \mu$, if $\lambda_{1}+\cdots+\lambda_{i} \geqslant \mu_{1}+\cdots+\mu_{i}$ for all $i \geqslant 1$.

Let $R=\mathbb{Q}(q, t)\left[s^{ \pm}\right], \lambda, \mu \in P_{+}(n)$ and

$$
\langle\lambda\rangle_{n ; q, t}:=\left(q^{\lambda_{1}} t^{n-1}, \ldots, q^{\lambda_{n-1}} t, q^{\lambda_{n}}\right)
$$

a spectral vector. Then the $\mathrm{BC}_{n}$-symmetric (Macdonald) interpolation polynomial

$$
\bar{P}_{\mu}^{*}(q, t, s)=\bar{P}_{\mu}^{*}(x ; q, t, s)
$$

is the unique polynomial in $\Lambda_{\mathrm{BC}(n)}$ of the form

$$
\bar{P}_{\mu}^{*}(q, t, s)=m_{\mu}^{W}+\sum_{\substack{\lambda \in P_{+}(n) \\ \lambda<\mu}} c_{\mu \lambda}(q, t, s) m_{\lambda}^{W}
$$


satisfying the vanishing conditions

$$
P_{\mu}\left(s\langle\lambda\rangle_{n ; q, t} ; q, t, s\right)=0 \quad \text { if } \mu \not \subset \lambda,
$$

see $[32,35]$. Since any triangular $\mathrm{BC}_{n}$-symmetric polynomial with leading term $m_{\mu}^{W}$ is uniquely determined by its values at $z\langle\lambda\rangle_{n ; q, t}$ for $\lambda<\mu$ and some arbitrary nonzero $z$, the above vanishing conditions in fact lead to an overdetermined linear system for the coefficients $c_{\mu \lambda}$. One of the main results of [32] is the actual existence of the interpolation polynomials. ${ }^{2}$

The interpolation polynomial $\bar{P}_{\mu}^{*}(q, t, s)$, whose top-degree term coincides with the Macdonald polynomial $P_{\mu}(q, t)$, satisfies the symmetries

$$
\bar{P}_{\mu}^{*}(x ; q, t, s)=\bar{P}_{\mu}^{*}(x ; 1 / q, 1 / t, 1 / s)=(-1)^{|\mu|} \bar{P}_{\mu}^{*}(-x ; q, t,-s)
$$

and

$$
\bar{P}_{\mu+\left(N^{n}\right)}^{*}(x ; q, t, s)=(-s)^{-n N} q^{-n\left(\begin{array}{c}
N \\
2
\end{array}\right)} \bar{P}_{\mu}^{*}\left(x ; q, t, s q^{N}\right) \prod_{i=1}^{n}\left(s x_{i}^{ \pm} ; q\right)_{N}
$$

for $N$ an arbitrary integer such that $N \geqslant-\mu_{n}$. Like the Macdonald polynomials, this can be used to extend the $\mathrm{BC}_{n}$ interpolation polynomials to arbitrary weights $\mu \in P(n)$ :

$$
\bar{P}_{\mu}^{*}(x ; q, t, s)=(-s)^{-n \mu_{n}} q^{-n\left(\begin{array}{c}
\mu_{n} \\
2
\end{array}\right)} \bar{P}_{\nu}^{*}\left(x ; q, t, s q^{\mu_{n}}\right) \prod_{i=1}^{n}\left(s x_{i}^{ \pm} ; q\right)_{\mu_{n}}
$$

where $\nu:=\left(\mu_{1}-\mu_{n}, \ldots, \mu_{n-1}-\mu_{n}, 0\right) \in P_{+}(n)$. Of course, for $\mu$ not dominant, i.e., for $\mu \notin P_{+}(n), \bar{P}_{\mu}^{*}(x ; q, t, s)$ is not a Laurent polynomial but a rational function in $x$.

The interpolation polynomials also admit a closed-form evaluation at $x=s\langle\mu\rangle_{n ; q, t}$

$$
\begin{aligned}
& P_{\mu}\left(s\langle\mu\rangle_{n ; q, t} ; q, t, s\right) \\
& \quad=\left(s q t^{n-1}\right)^{-|\mu|} q^{-2 n\left(\mu^{\prime}\right)} t^{n(\mu)} C_{\mu}^{-}(q ; q, t) C_{\mu}^{+}\left(s^{2} t^{2 n-2} ; q, t\right) \quad \text { for } \mu \in P_{+}(n),
\end{aligned}
$$

as well as a principal specialisation formula

$$
\begin{aligned}
& \bar{P}_{\mu}^{*}\left(z\langle 0\rangle_{n ; q, t} ; q, t, s\right) \\
& \quad=\left(-s t^{n-1}\right)^{-|\mu|} q^{-n\left(\mu^{\prime}\right)} t^{2 n(\mu)} \frac{C_{\mu}^{0}\left(t^{n}, s / z, z s t^{n-1} ; q, t\right)}{C_{\mu}^{-}(t ; q, t)} \quad \text { for } \mu \in P(n) .
\end{aligned}
$$

They may also be used to define generalised $q$-binomial coefficients [35, p. 81]

$$
\left[\begin{array}{l}
\lambda \\
\mu
\end{array}\right]_{q, t, s}:=\frac{\bar{P}_{\mu}^{*}\left(s t^{1-n}\langle\lambda\rangle_{n ; q, t} ; q, t, s t^{1-n}\right)}{\bar{P}_{\mu}^{*}\left(s t^{1-n}\langle\mu\rangle_{n ; q, t} ; q, t, s t^{1-n}\right)}
$$

where $\lambda, \mu$ are partitions and $n$ is an arbitrary integer such that $n \geqslant l(\lambda), l(\mu)$. The independence of $n$ readily follows from the fact that for any $\mu \in P(n+1)$,

$$
\bar{P}_{\mu}^{*}\left(x_{1}, \ldots, x_{n}, s ; q, t, s\right)= \begin{cases}\bar{P}_{\mu}^{*}\left(x_{1}, \ldots, x_{n} ; q, t, s t\right) & \text { if } \mu \in P_{+}(n), \\ 0 & \text { otherwise. }\end{cases}
$$

\footnotetext{
${ }^{2}$ Alternatively one may replace (4.12) by vanishing for $\lambda<\mu$ so that uniqueness and existence are immediate and then prove the extra vanishing conditions.
} 
Clearly, $\left[\begin{array}{l}\lambda \\ \mu\end{array}\right]_{q, t, s}=0$ unless $\mu \subset \lambda$ and $\left[\begin{array}{l}\lambda \\ 0\end{array}\right]_{q, t, s}=\left[\begin{array}{l}\lambda \\ \lambda\end{array}\right]_{q, t, s}=1$. By (4.13) it also follows that

$$
\left[\begin{array}{l}
\lambda \\
\mu
\end{array}\right]_{q, t, s}=\left[\begin{array}{l}
\lambda \\
\mu
\end{array}\right]_{1 / q, 1 / t, 1 / s}=\left[\begin{array}{l}
\lambda \\
\mu
\end{array}\right]_{q, t,-s},
$$

so that, in particular, the generalised binomial coefficients are a function of $s^{2}$ only. Less obvious is that the $q, t, s$-binomial coefficients satisfy conjugation symmetry [35, Corollary 4.3]

$$
\left[\begin{array}{l}
\lambda^{\prime} \\
\mu^{\prime}
\end{array}\right]_{t, q, s}=\left[\begin{array}{l}
\lambda \\
\mu
\end{array}\right]_{q, t, s^{-1}(q t)^{-1 / 2}} .
$$

As an immediate consequence of (4.14),

$$
\begin{aligned}
& {\left[\begin{array}{l}
\lambda+\left(N^{n}\right) \\
\mu+\left(N^{n}\right)
\end{array}\right]_{q, t, s}} \\
& =q^{-|\lambda / \mu| N} \frac{C_{\lambda}^{0}\left(s^{2} q^{2 N} t^{1-n}, q^{N+1} t^{n-1} ; q, t\right) C_{\mu}^{0}\left(s^{2} q^{N} t^{1-n}, q t^{n-1} ; q, t\right)}{C_{\mu}^{0}\left(s^{2} q^{2 N} t^{1-n}, q^{N+1} t^{n-1} ; q, t\right) C_{\lambda}^{0}\left(s^{2} q^{N} t^{1-n}, q t^{n-1} ; q, t\right)}\left[\begin{array}{c}
\lambda \\
\mu
\end{array}\right]_{q, t, s q^{N}} .
\end{aligned}
$$

Similarly, from (4.16) and (4.17),

$$
\left[\begin{array}{c}
\left(N^{n}\right) \\
\mu
\end{array}\right]_{q, t, s}=(-q)^{|\mu|} q^{n\left(\mu^{\prime}\right)} t^{n(\mu)} \frac{C_{\mu}^{0}\left(t^{n}, s^{2} q^{N} t^{1-n}, q^{-N} ; q, t\right)}{C_{\mu}^{-}(q, t ; q, t) C_{\mu}^{+}\left(s^{2} ; q, t\right)} .
$$

For $t=q$ the $\mathrm{BC}_{n}$ Macdonald interpolation polynomials simplify to the corresponding Schur functions, see, e.g., [33]. These are expressible as a simple Weyl-type determinant as

$$
\bar{P}_{\mu}^{*}(x ; q, q, s)=\frac{\operatorname{det}_{1 \leqslant i, j \leqslant n}\left(\bar{P}_{\left(\mu_{j}+n-j\right)}^{*}\left(x_{i} ; q, q, s\right)\right)}{\prod_{i=1}^{n} x_{i}^{1-n} \prod_{1 \leqslant i, j \leqslant n}\left(x_{i}-x_{j}\right)\left(x_{i} x_{j}-1\right)},
$$

where

$$
\bar{P}_{(k)}^{*}(z ; q, q, s)=(-s)^{-k} q^{\left(\begin{array}{c}
k \\
2
\end{array}\right)}\left(s z^{ \pm} ; q\right)_{k} .
$$

By (4.18) this yields the following determinantal expression for the generalised binomial coefficients when $t=q$ :

$$
\begin{aligned}
& {\left[\begin{array}{l}
\lambda \\
\mu
\end{array}\right]_{q, q, s}=(-1)^{|\nu|} q^{n\left(\nu^{\prime}\right)+n(\nu)-n(\kappa)-(n-2)|\nu|+(n-1)|\kappa|} \prod_{i=1}^{n} \frac{\left(s^{2} q^{2-2 n} ; q\right)_{\nu_{i}}}{(q ; q)_{\nu_{i}}\left(s^{2} q^{2-2 n} ; q\right)_{2 \nu_{i}}}} \\
& \quad \times \prod_{1 \leqslant i<j \leqslant n} \frac{\left(1-q^{\nu_{i}-\nu_{j}}\right)\left(1-s^{2} q^{\nu_{i}+\nu_{j}-2 n+2}\right)}{\left(1-q^{\kappa_{i}-\kappa_{j}}\right)\left(1-s^{2} q^{\kappa_{i}+\kappa_{j}-2 n+2}\right)} \operatorname{det}_{1 \leqslant i, j \leqslant n}\left(\left(s^{2} q^{\kappa_{i}-2 n+2}, q^{-\kappa_{i}} ; q\right)_{\nu_{j}}\right),
\end{aligned}
$$

where $n \geqslant l(\mu), l(\lambda)$ and $\kappa=\left(\kappa_{1}, \ldots, \kappa_{n}\right), \nu=\left(\nu_{1}, \ldots, \nu_{n}\right)$ are strict partitions defined by $\kappa_{i}:=\lambda_{i}+n-i, \nu_{i}:=\mu_{i}+n-i$.

\section{3 (Lifted) Koornwinder polynomials}

Let $\underline{t}:=\left(t_{0}, t_{1}, t_{2}, t_{3}\right)$ and $x:=\left(x_{1}, \ldots, x_{n}\right)$. For our purposes the most convenient way to define the Koornwinder polynomials

$$
K_{\lambda}(q, t ; \underline{t})=K_{\lambda}(x ; q, t ; \underline{t})
$$


for $\lambda \in P_{+}(n)$ - which are $\mathrm{BC}_{n}$-symmetric polynomials with coefficients in $\mathbb{Q}(q, t, \underline{t})$, see [20] is through Okounkov's binomial formula [32]:

$$
\begin{aligned}
K_{\lambda}(q, t ; \underline{t}):=\sum_{\mu \subset \lambda} & \left(t_{0} t^{n-1}\right)^{-|\lambda / \mu|} t^{n(\lambda / \mu)} \frac{C_{\mu}^{-}(t ; q, t) C_{\mu}^{+}\left(T^{2} ; q, t\right)}{C_{\lambda}^{-}(t ; q, t) C_{\lambda}^{+}\left(T^{2} ; q, t\right)} \\
& \times \frac{C_{\lambda}^{0}\left(t^{n}, t^{n-1} t_{0} t_{1}, t^{n-1} t_{0} t_{2}, t^{n-1} t_{0} t_{3} ; q, t\right)}{C_{\mu}^{0}\left(t^{n}, t^{n-1} t_{0} t_{1}, t^{n-1} t_{0} t_{2}, t^{n-1} t_{0} t_{3} ; q, t\right)}\left[\begin{array}{l}
\lambda \\
\mu
\end{array}\right]_{q, t, T} \bar{P}_{\mu}^{*}\left(q, t, t_{0}\right),
\end{aligned}
$$

where $T^{2}:=t^{2 n-2} t_{0} t_{1} t_{2} t_{3} / q$, and where we recall that $\left[\begin{array}{l}\lambda \\ \mu\end{array}\right]_{q, t, T}$ is a function of $T^{2}$ only. By (4.13) and (4.20) it follows that

$$
K_{\lambda}(x ; q, t ; \underline{t})=K_{\lambda}(x ; 1 / q, 1 / t ; 1 / \underline{t})=(-1)^{|\lambda|} K_{\lambda}(-x ; q, t ;-\underline{t}),
$$

where $1 / \underline{t}$ is shorthand for $\left(1 / t_{0}, 1 / t_{1}, 1 / t_{2}, 1 / t_{3}\right)$. One drawback of the above definition of the Koornwinder polynomials is that it hides the $S_{4}$-symmetry in the parameters $t_{0}, t_{1}, t_{2}, t_{3}$. It however follows from the connection coefficient formula for the interpolation polynomials [35, Theorem 3.12] combined with the multivariable $q$-Pfaff-Saalschütz summation [35, Theorem 4.2] that the obvious $S_{3}$-symmetry lifts to $S_{4}$, see [35] for details.

The main result for Koornwinder polynomial that we will be needing is Mimachi's Cauchy identity [28, Theorem 2.1]

$$
\sum_{\lambda \subset\left(m^{n}\right)}(-1)^{|\lambda|} K_{\left(m^{n}\right)-\lambda}(x ; q, t ; \underline{t}) K_{\lambda^{\prime}}(y ; t, q ; \underline{t})=\prod_{i=1}^{n} \prod_{j=1}^{m}\left(x_{i} y_{j}\right)^{-1}\left(x_{i}-y_{j}\right)\left(x_{i} y_{j}-1\right),
$$

where $y:=\left(y_{1}, \ldots, y_{m}\right)$.

As mentioned in the introduction, the lifted Koornwinder polynomial [35]

$$
K_{\lambda}(q, t, T ; \underline{t})=K_{\lambda}\left(x_{1}, x_{2}, \ldots ; q, t, T ; \underline{t}\right)
$$

is the unique symmetric function such that

$$
\tilde{K}_{\lambda}\left(x_{1}^{ \pm}, \ldots, x_{n}^{ \pm}, 0,0, \ldots ; q, t, t^{n} ; \underline{t}\right)= \begin{cases}K_{\lambda}\left(x_{1}, \ldots, x_{n} ; q, t ; \underline{t}\right) & \text { if } l(\lambda) \leqslant n, \\ 0 & \text { otherwise. }\end{cases}
$$

Some care is required when dealing with this function since the above equation requires $\underline{t}$ to be generic. Issues may arise for $\underline{t}$ such that $C_{\lambda}^{+}\left(t^{2 n-2} t_{0} t_{1} t_{2} t_{3} / q ; q, t\right)=0$. This for example happens for the parameter choice $\underline{t}=\left(1,-1, t^{1 / 2},-t^{1 / 2}\right)$ in which case it is important to specialise $T=t^{n}$ before specialising $\underline{t}$.

Recall the universal orthogonal, symplectic and special orthogonal characters, defined in (3.4), (3.5) and (3.7).

Lemma 4.1. We have

$$
\begin{aligned}
& \operatorname{sp}_{\lambda}=\tilde{K}_{\lambda}\left(q, q, T ; q^{1 / 2},-q^{1 / 2}, q,-q\right), \\
& \mathrm{o}_{\lambda}=\tilde{K}_{\lambda}\left(q, q, T ; 1,-1, q^{1 / 2},-q^{1 / 2}\right), \\
& \operatorname{so}_{\lambda}=\tilde{K}_{\lambda}\left(q, q, T ;-1,-q^{1 / 2}, q^{1 / 2}, q\right) .
\end{aligned}
$$

Proof. Given that the lifted Koornwinder polynomials are the unique symmetric functions satisfying (4.28) and the universal characters $\mathrm{sp}_{\lambda}, \mathrm{o}_{\lambda}$ and $\mathrm{so}_{\lambda}$ are the unique symmetric functions satisfying (3.6) (in the case of $\mathrm{sp}_{\lambda}$ and $\mathrm{o}_{\lambda}$ ) or (3.8) (in the case of $\mathrm{so}_{\lambda}$ ), it suffices to show that

$$
\begin{aligned}
& \operatorname{sp}_{2 n, \lambda}\left(x_{1}, \ldots, x_{n}\right)=K_{\lambda}\left(x_{1}, \ldots, x_{n} ; q, q ; q^{1 / 2},-q^{1 / 2}, q,-q\right), \\
& \mathrm{O}_{2 n, \lambda}\left(x_{1}, \ldots, x_{n}\right)=K_{\lambda}\left(x_{1}, \ldots, x_{n} ; q, q ; 1,-1, q^{1 / 2},-q^{1 / 2}\right), \\
& \mathrm{so}_{2 n+1, \lambda}\left(x_{1}, \ldots, x_{n}\right)=K_{\lambda}\left(x_{1}, \ldots, x_{n} ; q, q ;-1,-q^{1 / 2}, q^{1 / 2}, q\right) .
\end{aligned}
$$


All three of the above identities follow directly from [42, Section 2.6]. For example, for $t=q$ and $\underline{t}=\left(q^{1 / 2},-q^{1 / 2}, q,-q\right)$ the Koornwinder density $\Delta(z ; q, t ; \underline{t})$ simplifies to the standard $\mathrm{C}_{n}$ density

$$
\Delta_{\mathrm{C}}(z)=\prod_{i=1}^{n}\left(-z_{i}^{-2}\right)\left(1-z_{i}^{2}\right)^{2} \prod_{1 \leqslant i<j \leqslant n} z_{i}^{-2}\left(1-z_{i} / z_{j}\right)^{2}\left(1-z_{i} z_{j}\right)^{2}
$$

for which

$$
\frac{1}{2^{n} n !(2 \pi \mathrm{i})^{n}} \int_{\mathbb{T}^{n}} \operatorname{sp}_{2 n, \lambda}(z) \operatorname{sp}_{2 n, \mu}(z) \Delta_{\mathrm{C}}(z) \frac{\mathrm{d} z_{1}}{z_{1}} \cdots \frac{\mathrm{d} z_{n}}{z_{n}}=\delta_{\lambda, \mu} .
$$

Similar reductions to Weyl-type orthogonality relations hold for the other two cases.

\subsection{Elliptic interpolation functions}

The $\left(\mathrm{BC}_{n}\right.$-symmetric) elliptic interpolation functions $R_{\mu}^{*}(a, b ; q, t ; p)[5,36,39]$ are an elliptic analogue of the $\mathrm{BC}_{n}$-symmetric interpolation polynomials $\bar{P}_{\mu}^{*}(q, t, s)$. Although they satisfy analogous vanishing conditions, their definition is more complicated. Below we follow the characterisation of these function given in [36].

$\mathrm{A} \mathrm{BC}_{n}$-symmetric theta function of degree $m$ is a $\mathrm{BC}_{n}$-symmetric meromorphic function $f$ on $\left(\mathbb{C}^{*}\right)^{n}$ such that

$$
f\left(p x_{1}, x_{2}, \ldots, x_{n}\right)=\left(1 / p x_{1}^{2}\right)^{m} f\left(x_{1}, x_{2}, \ldots, x_{n}\right) .
$$

For example, $\prod_{i=1}^{n} \theta\left(u x_{i}^{ \pm} ; p\right)$ for $u \in \mathbb{C}^{*}$ is a $\mathrm{BC}_{n^{-}}$-symmetric theta function of degree 1 .

Given two partitions $\lambda, \mu \subset\left(m^{n}\right)$ such that $\lambda \neq \mu$ let

$$
l_{0}=\max \left\{i: \lambda_{i} \neq \mu_{i}\right\} \quad \text { and } \quad l_{1}=\max \left\{i: \lambda_{i}=m\right\},
$$

where $l_{1}=0$ if $\lambda_{1}<m$. Given such $l_{1}$ and $l_{2}$, further let

$$
l= \begin{cases}l_{1} & \text { if } \lambda_{l_{0}}<\mu_{l_{0}} \\ l_{0} & \text { otherwise }\end{cases}
$$

Note that $l=n$ if and only if $\lambda_{n}>\mu_{n}$ and $l=0$ if $\lambda_{i}<\mu_{i}$ for all $1 \leqslant i \leqslant n$.

Now fix a nonnegative integer $m$ and partition $\mu \subset\left(m^{n}\right)$. Then the interpolation theta function

$$
P_{\mu}^{*(m)}\left(x_{1}, \ldots, x_{n} ; a, b ; q, t ; p\right)
$$

is the unique $\mathrm{BC}_{n}$-symmetric theta function of degree $m$ such that for all $\lambda \subset\left(m^{n}\right), \lambda \neq \mu,{ }^{3}$

$$
P_{\mu}^{*(m)}\left(b q^{m-\lambda_{1}}, \ldots, b q^{m-\lambda_{l}} t^{l-1}, a q^{\lambda_{l+1}} t^{n-l-1}, \ldots, a q^{\lambda_{n}} ; a, b ; q, t ; p\right)=0
$$

and

$$
\begin{aligned}
& P_{\mu}^{*(m)}\left(z\langle 0\rangle_{n ; q, t} ; a, b ; q, t ; p\right) \\
& \quad=C_{\left(m^{n}\right)}^{0}\left(t^{n-1} b z, b / z ; q, t ; p\right) \Delta_{\mu}^{0}\left(a q^{-m} t^{n-1} / b \mid t^{n-1} a z, a / z ; q, t ; p\right) .
\end{aligned}
$$

\footnotetext{
${ }^{3}$ It is assumed that the parameters $a, b, q, t$ of $P_{\mu}^{*(m)}(a, b ; q, t ; p)$ are chosen to be generic, and similarly for $z$ in the normalisation (4.29).
} 
Since the interpolation theta functions satisfy

$$
P_{\mu}^{*(m+1)}(x ; a, b ; q, t ; p)=P_{\mu}^{*(m)}(x ; a, b q ; q, t ; p) \prod_{i=1}^{n} \theta\left(b x_{i}^{ \pm} ; q, p\right), \quad \mu \subset\left(m^{n}\right),
$$

(and thus $\left.P_{0}^{*(m)}(x ; a, b ; q, t ; p)=\prod_{i=1}^{n}\left(b x_{i}^{ \pm} ; q, p\right)_{m}\right)$, the ratio

$$
R_{\mu}^{*}(a, b ; q, t ; p):=\frac{P_{\mu}^{*(m)}\left(a, b q^{-m} ; q, t ; p\right)}{P_{0}^{*(m)}\left(a, b q^{-m} ; q, t ; p\right)},
$$

is independent of $m$ (provided $m \geqslant \mu_{1}$ ) and a degree-0 (hence elliptic) $\mathrm{BC}_{n}$-symmetric theta function. The elliptic interpolation functions satisfy vanishing conditions analogous to (4.12):

$$
R_{\mu}^{*}\left(a\langle\lambda\rangle_{n ; q, t} ; a, b ; q, t ; p\right)=0 \quad \text { if } \mu \not \subset \lambda
$$

for $\lambda \in P_{+}(n)$. They also satisfy

$$
R_{\mu+\left(1^{n}\right)}^{*}(x ; a, b ; q, t ; p)=R_{\mu}^{*}(x ; a q, b / q ; q, t ; p) \prod_{i=1}^{n} \frac{\theta\left(a x_{i}^{ \pm} ; p\right)}{\theta\left(p q x_{i}^{ \pm} / b ; p\right)},
$$

so that, once again, they can be extended to all $\mu \in P(n)$ via

$$
R_{\mu}^{*}(x ; a, b ; q, t ; p)=R_{\nu}^{*}\left(x ; a q^{\mu_{n}}, b q^{-\mu_{n}} ; q, t ; p\right) \prod_{i=1}^{n} \frac{\left(a x_{i}^{ \pm} ; q, p\right)_{\mu_{n}}}{\left(p q x_{i}^{ \pm} / b ; q, p\right)_{\mu_{n}}},
$$

where $\nu:=\left(\mu_{1}-\mu_{n}, \ldots, \mu_{n-1}-\mu_{n}, 0\right)$. We further extend this to pairs of weights $\boldsymbol{\mu}=$ $\left(\mu^{(1)}, \mu^{(2)}\right) \in P(n) \times P(n)$ as

$$
R_{\mu}^{*}(x ; a, b ; t ; p, q):=R_{\mu^{(1)}}^{*}(x ; a, b ; p, t ; q) R_{\mu^{(2)}}^{*}(x ; a, b ; q, t ; p) .
$$

In the limit the elliptic interpolation functions simplify to the $\mathrm{BC}_{n}$-symmetric interpolation polynomials:

$$
\lim _{p \rightarrow 0} R_{\mu}^{*}\left(s, b p^{\alpha} ; q, t ; p\right)=\left(-s t^{n-1}\right)^{|\mu|} q^{n\left(\mu^{\prime}\right)} t^{-2 n(\mu)} \frac{C_{\mu}^{-}(t ; q, t)}{C_{\mu}^{0}\left(t^{n} ; q, t\right)} \bar{P}_{\mu}^{*}(q, t, s),
$$

for $0<\alpha<1$ and $\mu \in P(n)$.

In the following we need a number of identities for the $\mathrm{BC}_{n}$ interpolation function from [36]. By (4.29) and (4.31),

$$
R_{\mu}^{*}\left(z\langle 0\rangle_{n ; q, t} ; a, b ; q, t ; p\right)=\Delta_{\mu}^{0}\left(a t^{n-1} / b \mid t^{n-1} a z, a / z ; q, t ; p\right)
$$

for $\mu \in P(n)$, which generalises (4.17). The elliptic analogue of (4.16) is given by

$$
\begin{aligned}
& R_{\mu}^{*}\left(a\langle\mu\rangle_{n ; q, t} ; a, b ; q, t ; p\right) \\
& \quad=\frac{1}{\Delta_{\mu}^{0}\left(a^{2} t^{2 n-2} \mid a b t^{n-1} ; q, t ; p\right) \Delta_{\mu}\left(a t^{n-1} / b \mid t^{n} ; q, t ; p\right)} \frac{C_{\mu}^{+}\left(a^{2} t^{2 n-2} ; q, t ; p\right)}{C_{\mu}^{+}\left(a t^{n-1} / b ; q, t ; p\right)},
\end{aligned}
$$

for $\mu \in P_{+}(n)$ and the analogue of (4.19) by

$$
\begin{aligned}
& R_{\mu}^{*}\left(x_{1}, \ldots, x_{n}, a ; a, b ; q, t ; p\right) \\
& \quad= \begin{cases}\frac{C_{\mu}^{0}\left(t^{n}, a p q / b t ; q, t ; p\right)}{C_{\mu}^{0}\left(t^{n+1}, a p q / b ; q, t ; p\right)} R_{\mu}^{*}\left(x_{1}, \ldots, x_{n} ; a t, b ; q, t ; p\right) & \text { if } \mu \in P_{+}(n), \\
0 & \text { otherwise, }\end{cases}
\end{aligned}
$$


for $\mu \in P(n+1)$. We also require the symmetry

$$
R_{\mu}^{*}(x ; a, b ; q, t ; p)=\left(a q t^{n-1} / b\right)^{|\mu|} q^{2 n\left(\lambda^{\prime}\right)} t^{-2 n(\mu)} R_{\mu}^{*}\left(x p^{1 / 2} ; a p^{1 / 2}, b p^{1 / 2} ; q, t ; p\right),
$$

for $\mu \in P(n)$.

Given partitions $\lambda, \mu$ and $n$ an arbitrary integer such that $n \geqslant l(\lambda), l(\mu)$, the elliptic analogue of the binomial coefficient $\left[\begin{array}{c}\lambda \\ \mu\end{array}\right]_{q, t, s}$ is defined as [36]

$$
\begin{aligned}
\left\langle\begin{array}{l}
\lambda \\
\mu
\end{array}\right\rangle_{[a, b] ; q, t ; p}: & =\frac{\Delta_{\lambda}^{0}(a \mid b ; q, t ; p)}{\Delta_{\mu}^{0}(a \mid b ; q, t ; p)} \frac{C_{\mu}^{+}(a ; q, t ; p)}{C_{\mu}^{+}(a / b ; q, t ; p)} \frac{R_{\mu}^{*}\left(a^{1 / 2} t^{1-n}\langle\lambda\rangle_{q, t ; n} ; a^{1 / 2} t^{1-n}, b / a^{1 / 2} ; q, t ; p\right)}{R_{\mu}^{*}\left(a^{1 / 2} t^{1-n}\langle\mu\rangle_{q, t ; n} ; a^{1 / 2} t^{1-n}, b / a^{1 / 2} ; q, t ; p\right)} \\
& =\Delta_{\lambda}^{0}(a \mid b ; q, t ; p) \Delta_{\mu}\left(a / b \mid t^{n} ; q, t ; p\right) R_{\mu}^{*}\left(a^{1 / 2} t^{1-n}\langle\lambda\rangle_{q, t ; n} ; a^{1 / 2} t^{1-n}, b / a^{1 / 2} ; q, t ; p\right)
\end{aligned}
$$

where the equality of the two expressions on the right follows from (4.35). Since

$$
R_{\mu}^{*}(x ; a, b ; q, t ; p)=R_{\mu}^{*}(-x ;-a,-b ; q, t ; p),
$$

the elliptic binomial coefficients are a function of $a$ (as opposed to $a^{1 / 2}$ ) so that no choice of branch is required. Moreover, by (4.36) they are independent of the choice of $n$ on the right as long as $n$ is sufficiently large, and by (4.30), they vanish unless $\mu \subset \lambda$. Since, for $\mu \subset \lambda$, $\Delta_{\lambda}^{0}(a \mid b ; q, t ; p) /\left.\Delta_{\mu}^{0}(a \mid b ; q, t ; p)\right|_{b=1}=\delta_{\lambda \mu}$ (and since no poles are hit by taking $b=1$ in any of the other terms in the definition of the elliptic binomial coefficients)

$$
\left\langle\begin{array}{l}
\lambda \\
\mu
\end{array}\right\rangle_{[a, 1] ; q, t ; p}=\delta_{\lambda \mu}
$$

Furthermore, since $R_{0}^{*}=1$,

$$
\left\langle\begin{array}{l}
\lambda \\
0
\end{array}\right\rangle_{[a, b] ; q, t ; p}=\Delta_{\lambda}^{0}(a \mid b ; q, t ; p) \quad \text { and } \quad\left\langle\begin{array}{l}
\lambda \\
\lambda
\end{array}\right\rangle_{[a, b] ; q, t ; p}=\frac{C_{\lambda}^{+}(a ; q, t ; p)}{C_{\lambda}^{+}(a / b ; q, t ; p)} .
$$

By (4.34), also for $\lambda=\left(N^{n}\right)$ the binomial coefficients factor:

$$
\left\langle\begin{array}{c}
\left(N^{n}\right) \\
\mu
\end{array}\right\rangle_{[a, b] ; q, t ; p}=\Delta_{\left(N^{n}\right)}^{0}(a \mid b ; q, t ; p) \Delta_{\mu}\left(a / b \mid t^{n}, a q^{N} t^{1-n}, q^{-N} ; q, t ; p\right) .
$$

The elliptic binomial coefficients satisfy a large number of symmetries and identities, and for a complete list of these the reader is referred to the original papers [36, 38, 39] or the survey [45]. Here we state a selection of result needed later.

The most important summation for elliptic binomial coefficients is the convolution-type formula

$$
\begin{gathered}
\sum_{\nu \subset \mu \subset \lambda} \Delta_{\mu}^{0}(a \mid d, e ; q, t ; p)\left\langle\begin{array}{l}
\lambda \\
\mu
\end{array}\right\rangle_{[a b, b] ; q, t ; p}\left\langle\begin{array}{l}
\mu \\
\nu
\end{array}\right\rangle_{[a, c] ; q, t ; p} \\
=\frac{\Delta_{\lambda}^{0}(a b \mid b d, b e ; q, t ; p)}{\Delta_{\nu}^{0}(a / c \mid b d, b e ; q, t ; p)}\left\langle\begin{array}{l}
\lambda \\
\nu
\end{array}\right\rangle_{[a b, b c] ; q, t ; p},
\end{gathered}
$$

provided $b c d e=a p q$. For $b c=1$ the right-hand side trivialises by (4.38), resulting in the inversion relation

$$
\sum_{\nu \subset \mu \subset \lambda}\left\langle\begin{array}{l}
\lambda \\
\mu
\end{array}\right\rangle_{[a b, b] ; q, t ; p}\left\langle\begin{array}{l}
\mu \\
\nu
\end{array}\right\rangle_{[a, 1 / b] ; q, t ; p}=\delta_{\lambda \nu}
$$


By (4.39) and (4.40) it follows that the special case $\lambda=\left(N^{n}\right)$ and $\nu=0$ of (4.41) corresponds to the $(\mathrm{B}) \mathrm{C}_{n}$-analogue of the elliptic Jackson sum (2.18), see, e.g., [5, 17, 36, 43, 52].

Two important symmetries we will rely on in Section 7 are the reciprocity and conjugation symmetries

$$
\begin{aligned}
\left\langle\begin{array}{l}
\lambda \\
\mu
\end{array}\right\rangle_{[1 / a, 1 / b] ; 1 / q, 1 / t ; p} & =\left\langle\begin{array}{l}
\lambda^{\prime} \\
\mu^{\prime}
\end{array}\right\rangle_{[1 / a q t, 1 / b] ; t, q ; p} \\
& =(-a q)^{|\mu|-2|\lambda|} q^{n\left(\mu^{\prime}\right)-4 n\left(\lambda^{\prime}\right)} t^{4 n(\lambda)-n(\mu)}\left\langle\begin{array}{l}
\lambda \\
\mu
\end{array}\right\rangle_{[a, b / p] ; q, t ; p} .
\end{aligned}
$$

Finally, as follows from (4.18) and (4.33), in the limit the elliptic binomial coefficients reduce to the binomial coefficients (4.18):

$$
\lim _{p \rightarrow 0}\left\langle\begin{array}{l}
\lambda \\
\mu
\end{array}\right\rangle_{\left[s^{2}, b / p^{1 / 2}\right] ; q, t ; p}=\left(s^{2} q\right)^{|\lambda / \mu|} q^{2 n\left(\lambda^{\prime} / \mu^{\prime}\right)} t^{-2 n(\lambda / \mu)} C_{\mu}^{+}\left(s^{2} ; q, t\right)\left[\begin{array}{l}
\lambda \\
\mu
\end{array}\right]_{q, t, s} .
$$

In Sections 5 and 7 we also use the $p, q$-symmetric variant of the elliptic binomial coefficients, defined as

$$
\left\langle\begin{array}{l}
\boldsymbol{\lambda} \\
\boldsymbol{\mu}
\end{array}\right\rangle_{[a, b] ; t ; p, q}:=\left\langle\begin{array}{l}
\lambda^{(1)} \\
\mu^{(1)}
\end{array}\right\rangle_{[a, b] ; p, t ; q}\left\langle\begin{array}{l}
\lambda^{(2)} \\
\mu^{(2)}
\end{array}\right\rangle_{[a, b] ; q, t ; p} .
$$

\section{$5 \quad$ Elliptic beta integrals and interpolation kernels}

\subsection{Elliptic beta integrals}

Let $n$ be a positive integer, $m$ an even nonnegative integer and $p, q, t, t_{0}, \ldots, t_{m-1} \in \mathbb{C}^{*}$ such that $|p|,|q|<1$. Then the elliptic Dixon (or type I) and Selberg (or type II) densities are defined as $[38,40,45]$

$$
\Delta_{\mathrm{D}}\left(z ; t_{0}, \ldots, t_{m-1} ; p, q\right):=\kappa_{n} \prod_{1 \leqslant i<j \leqslant n} \frac{1}{\Gamma_{p, q}\left(z_{i}^{ \pm} z_{j}^{ \pm}\right)} \prod_{i=1}^{n} \frac{\prod_{r=0}^{m-1} \Gamma_{p, q}\left(t_{r} z_{i}^{ \pm}\right)}{\Gamma_{p, q}\left(z_{i}^{ \pm 2}\right)}
$$

and

$$
\Delta_{\mathrm{S}}\left(z ; t_{0}, \ldots, t_{m-1} ; t ; p, q\right):=\kappa_{n} \prod_{1 \leqslant i<j \leqslant n} \frac{\Gamma_{p, q}\left(t z_{i}^{ \pm} z_{j}^{ \pm}\right)}{\Gamma_{p, q}\left(z_{i}^{ \pm} z_{j}^{ \pm}\right)} \prod_{i=1}^{n} \frac{\Gamma_{p, q}(t) \prod_{r=0}^{m-1} \Gamma_{p, q}\left(t_{r} z_{i}^{ \pm}\right)}{\Gamma_{p, q}\left(z_{i}^{ \pm 2}\right)}
$$

where

$$
\kappa_{n}=\kappa_{n}(p, q):=\frac{(p ; p)_{\infty}^{n}(q ; q)_{\infty}^{n}}{2^{n} n !(2 \pi \mathrm{i})^{n}} .
$$

The actual elliptic Dixon and Selberg integral evaluations are given by [10, 11, 38, 45]

$$
\int_{C} \Delta_{\mathrm{D}}\left(z ; t_{0}, \ldots, t_{2 n+3} ; p, q\right) \frac{\mathrm{d} z_{1}}{z_{1}} \cdots \frac{\mathrm{d} z_{n}}{z_{n}}=\prod_{0 \leqslant r<s \leqslant 2 n+3} \Gamma_{p, q}\left(t_{r} t_{s}\right)
$$

for $t_{0} \cdots t_{2 n+3}=p q$, and

$$
\begin{aligned}
S_{n}\left(t_{0}, \ldots, t_{5} ; t ; p, q\right) & :=\int_{C} \Delta_{\mathrm{S}}\left(z ; t_{0}, \ldots, t_{5} ; t ; p, q\right) \frac{\mathrm{d} z_{1}}{z_{1}} \cdots \frac{\mathrm{d} z_{n}}{z_{n}} \\
& =\prod_{i=1}^{n}\left(\Gamma_{p, q}\left(t^{i}\right) \prod_{0 \leqslant r<s \leqslant 5} \Gamma_{p, q}\left(t^{i-1} t_{r} t_{s}\right)\right),
\end{aligned}
$$


for $t^{2 n-2} t_{0} \cdots t_{5}=p q$. In both integrals $C$ is a deformation of $\mathbb{T}^{n}$, separating the double geometric progressions of poles of the integrands tending to zero from those tending to infinity. For (5.2) we must also have that $t C$ is contained in $C$. When $\left|u_{r}\right|,\left|t_{r}\right|<1$ for all $r$ as well as $|t|<1$ in the elliptic Selberg case, we may simply take $C=\mathbb{T}^{n}{ }^{4}$ For $n=1$ the integrals (5.1) and (5.2) coincide and correspond to Spiridonov's elliptic beta integral [49].

Given a $\mathrm{BC}_{n}$-symmetric function $f$, we define its elliptic Selberg average by

$$
\langle f\rangle_{t_{0}, \ldots, t_{5} ; t ; p, q}^{(n)}:=\frac{1}{S_{n}\left(t_{0}, \ldots, t_{5} ; t ; p, q\right)} \int_{C} f(z) \Delta_{\mathrm{S}}\left(z ; t_{0}, \ldots, t_{5} ; t ; p, q\right) \frac{\mathrm{d} z_{1}}{z_{1}} \cdots \frac{\mathrm{d} z_{n}}{z_{n}},
$$

where $C=C_{f}$ is as above and $t^{2 n-2} t_{0} \cdots t_{5}=p q$.

\subsection{The interpolation kernel}

Let $x, y \in\left(\mathbb{C}^{*}\right)^{n}$ and $c, p, q, t \in \mathbb{C}^{*}$ such that $|p|,|q|<1$. Then the interpolation kernel $\mathcal{K}_{c}(x ; y ; t ; p, q)$ is a meromorphic $\mathrm{BC}_{n}$-symmetric function in both $x$ and $y$, satisfying

$$
\mathcal{K}_{c}(x ; y ; t ; p, q)=\mathcal{K}_{c}(x ; y ; t ; q, p)=\mathcal{K}_{c}(y ; x ; t ; p, q)=\mathcal{K}_{-c}(-x ; y ; t ; p, q)
$$

such that $[40]$

$$
\mathcal{K}_{c}\left(x ; a\langle\boldsymbol{\mu}\rangle_{n ; t ; p, q} / c ; t ; p, q\right)=R_{\boldsymbol{\mu}}^{*}(x ; a, b ; q, t ; p) \prod_{i=1}^{n}\left(\frac{p q}{a b}\right)^{2 \mu_{i}^{(1)} \mu_{i}^{(2)}} \frac{\Gamma_{p, q}\left(a x_{i}^{ \pm}, b x_{i}^{ \pm}\right)}{\Gamma_{p, q}\left(t^{i}, a b t^{n-i}\right)},
$$

where $c^{2}=a b t^{n-1}$ and

$$
\langle\boldsymbol{\mu}\rangle_{n ; t ; p, q}:=\left(p^{\mu_{1}^{(1)}} q^{\mu_{1}^{(2)}} t^{n-1}, \ldots, p^{\mu_{n-1}^{(1)}} q^{\mu_{n-1}^{(2)}} t, p^{\mu_{n}^{(1)}} q^{\mu_{n}^{(2)}}\right)
$$

is an elliptic spectral vector. The interpolation kernel may recursively be defined using the initial conditions

$$
\mathcal{K}_{c}(-;-; t, p, q)=1 \quad \text { or } \quad \mathcal{K}_{c}\left(x_{1} ; y_{1} ; t, p, q\right)=\frac{\Gamma_{p, q}\left(c x_{1}^{ \pm} y_{1}^{ \pm}\right)}{\Gamma_{p, q}\left(t, c^{2}\right)}
$$

and branching rule

$$
\begin{aligned}
& \mathcal{K}_{c}(x ; y ; t ; p, q)=\frac{\prod_{i=1}^{n} \Gamma_{p, q}\left(c y_{n}^{ \pm} x_{i}^{ \pm}\right)}{\Gamma_{p, q}^{n}(t) \Gamma_{p, q}\left(c^{2}\right) \prod_{1 \leqslant i<j \leqslant n} \Gamma_{p, q}\left(t x_{i}^{ \pm} x_{j}^{ \pm}\right)} \\
& \quad \times \int_{C} \mathcal{K}_{c / t^{1 / 2}}(z ; \hat{y} ; t ; p, q) \Delta_{\mathrm{D}}\left(t^{1 / 2} x^{ \pm}, p q y_{n}^{ \pm} / c t^{1 / 2} ; z ; p, q\right) \frac{\mathrm{d} z_{1}}{z_{1}} \cdots \frac{\mathrm{d} z_{n-1}}{z_{n-1}},
\end{aligned}
$$

for $x, y \in\left(\mathbb{C}^{*}\right)^{n}, \hat{y}=\left(y_{1}, \ldots, y_{n-1}\right), z \in\left(\mathbb{C}^{*}\right)^{n-1}$ and a suitable subset of the parameter space. By (5.3), making the substitution

$$
\left(c, t^{1 / 2}, x\right) \mapsto\left(-c,-t^{1 / 2},-x\right)
$$

(and also negating the integration variables on the right) leaves (5.6) unchanged. Hence there is no need to fix a branch of $t^{1 / 2}$. We further note that the symmetry of the kernel in $y$ is not manifest from the recursive definition, but follows from a similar such symmetry for the "formal interpolation kernel" $K_{c}(x ; y ; q, t ; p)$ defined in [40] as a generalisation of the connection coefficients identity for $R_{\mu}^{*}(x ; a, b ; q, t)$. (By (5.5), for $n=2$ the symmetry is an immediate consequence of a special case of the elliptic integral transformation [38, Theorem 4.1].)

\footnotetext{
${ }^{4}$ For a more detailed analysis of the contours and potential issues, including existence, see the appendix of [38].
} 


\subsection{The dual Littlewood kernel}

Following [40] we consider two further kernels, known as the dual Littlewood kernel and Kawanaka kernel. For the dual Littlewood kernel, $\mathcal{L}_{c}^{\prime}(x ; t ; p, q)$, let $x \in\left(\mathbb{C}^{*}\right)^{n}$ and $c, p, q, t, u, v \in \mathbb{C}^{*}$ such that $c^{4} u v=p$ and $|p|,|q|<1$. Then [40]

$$
\begin{aligned}
\mathcal{L}_{c}^{\prime}(x ; t ; p, q):= & \frac{1}{\prod_{i=1}^{n} \Gamma_{p, q}\left(c u x_{i}^{ \pm}, c v x_{i}^{ \pm}\right)} \\
& \times \int \mathcal{K}_{c}(z ; x ; t ; p, q) \Delta_{\mathrm{S}}\left(z ; u, v ; t ; p, q^{2}\right) \frac{\mathrm{d} z_{1}}{z_{1}} \cdots \frac{\mathrm{d} z_{n}}{z_{n}} .
\end{aligned}
$$

As shown in [40, Corollary 7.3], the right-hand side depends on the product of $u$ and $v$ only so that the dual Littlewood kernel is well-defined. Like the interpolation kernel $\mathcal{K}_{c}, \mathcal{L}_{-c}^{\prime}(-x ; t, p, q)=$ $\mathcal{L}_{c}^{\prime}(-x ; t, p, q)$. It further satisfies the symmetry

$$
\mathcal{L}_{c}^{\prime}(x ; p q / t ; p, q)=\mathcal{L}_{c}^{\prime}(x ; t ; p, q) \prod_{1 \leqslant i<j \leqslant n} \Gamma_{p, q}\left(t x_{i}^{ \pm} x_{j}^{ \pm}\right),
$$

and factorisation formula

$$
\mathcal{L}_{(p / q t)^{1 / 2}}^{\prime}\left(x ; t^{2} ; p^{2}, q^{2}\right)=\Gamma_{p^{2}, q^{2}}^{n}(p / q t) \prod_{i=1}^{n} \Gamma_{p^{2}, q^{4}}\left(p q x_{i}^{ \pm 2} / t\right) \prod_{1 \leqslant i<j \leqslant n} \Gamma_{p^{2}, q^{2}}\left(p q x_{i}^{ \pm} x_{j}^{ \pm} / t\right) .
$$

Evaluating (5.7) at $x=a\langle\boldsymbol{\mu}\rangle_{n ; t ; p, q} / c$ and using (5.4) as well as the simple relations $\Gamma_{p, q}(z)=$ $\Gamma_{p, q^{2}}(z, z q)$ and

$$
\frac{\Gamma_{p, q}\left(z p^{k} q^{\ell}\right)}{\Gamma_{p, q}(z)}=(-z)^{-k \ell} p^{-\ell\left(\begin{array}{c}
k \\
2
\end{array}\right)} q^{-k\left(\begin{array}{l}
\ell \\
2
\end{array}\right)}(z ; p, q)_{k}(z ; q, p)_{\ell},
$$

we find

$$
\frac{\mathcal{L}_{c}^{\prime}\left(a\langle\boldsymbol{\mu}\rangle_{n ; t ; p, q} / c ; t ; p, q\right)}{\mathcal{L}_{c}^{\prime}\left(a\langle\mathbf{0}\rangle_{n ; t ; p, q} / c ; t ; p, q\right)}=\left(u v t^{n-1}\right)^{2 \sum_{i=1}^{n} \mu_{i}^{(1)} \mu_{i}^{(2)}} \frac{\left\langle R_{\boldsymbol{\mu}}^{*}(z ; a, b ; q, t ; p)\right\rangle_{a, a q, b, b q, u, v ; t ; p, q^{2}}^{(n)}}{\Delta_{\boldsymbol{\mu}}^{0}\left(a t^{n-1} / b \mid a u t^{n-1}, a v t^{n-1} ; t ; p, q\right)},
$$

where, as before, $c^{4} u v=p$ and $c^{2}=a b t^{n-1}$. Replacing $(p, q, t) \mapsto\left(p^{2}, q^{2}, t^{2}\right)$ and then specialising $c=(p / q t)^{1 / 2}$ the left-hand side factors thanks to (5.8). By (5.9) this yields

$$
\begin{aligned}
\left\langle R_{\boldsymbol{\mu}}^{*}(z ;\right. & \left.\left.a, b ; t^{2} ; p^{2}, q^{2}\right)\right\rangle_{a, a q^{2}, b, b q^{2}, u, v ; t^{2} ; p^{2}, q^{4}}^{(n)} \\
= & \Delta_{\boldsymbol{\mu}}^{0}\left(a t^{2 n-2} / b \mid a^{2} q^{2} t^{2 n-2}, a u t^{2 n-2}, a v t^{2 n-2} ; t^{2} ; p^{2}, q^{2}\right) \\
& \times \frac{C_{\boldsymbol{\mu}}^{-}\left(p q t ; t^{2} ; p^{2}, q^{2}\right) C_{\boldsymbol{\mu}}^{+}\left(a^{2} q^{2} t^{4 n-4} ; t^{2} ; p^{2}, q^{2}\right)}{C_{(2,1) \boldsymbol{\mu}^{2}}^{0}\left(a^{2} q^{4} t^{4 n-2} ; t^{2} ; p^{2}, q^{4}\right)},
\end{aligned}
$$

where $u v=(q t)^{2}$ and $a b q t^{2 n-1}=p$. For later convenience we interchange $p$ and $q$ (and $\left.\left(\mu^{(1)}, \mu^{(2)}\right) \mapsto\left(\mu^{(2)}, \mu^{(1)}\right)\right)$. Using the $p, q$-symmetry of the Selberg average, and finally replacing $(a, b, u, v) \mapsto(q / b t, q / a t, p t v, p t / v)$, (5.10) may equivalently be stated as in our next theorem.

Theorem 5.1. For $\boldsymbol{\mu} \in P_{+}(n) \times P_{+}(n)$ and $a, b, v, p, q, t \in \mathbb{C}^{*}$ such that $a b=p q t^{2 n-3}$ and $|p|,|q|<1$,

$$
\begin{aligned}
& \left\langle R_{\boldsymbol{\mu}}^{*}\left(z ; q / b t, q / a t ; t^{2} ; p^{2}, q^{2}\right)\right\rangle_{q / a t, p^{2} q / a t, q / b t, p^{2} q / b t, p t v, p t / v ; t^{2} ; p^{4}, q^{2}}^{(n)} \\
& \quad=\Delta_{\boldsymbol{\mu}}^{0}\left(a^{2} t / p q \mid a^{2} t^{2-2 n}, a t v^{ \pm} ; t^{2} ; p^{2}, q^{2}\right) \frac{C_{\boldsymbol{\mu}}^{-}\left(p q t ; t^{2} ; p^{2}, q^{2}\right) C_{\boldsymbol{\mu}}^{+}\left(a^{2} ; t^{2} ; p^{2}, q^{2}\right)}{C_{(1,2) \boldsymbol{\mu}^{2}}^{0}\left((a p t)^{2} ; t^{2} ; p^{4}, q^{2}\right)} .
\end{aligned}
$$


Eliminating $b$, let $f_{\mu}(a, v ; t ; p, q)$ denote the left-hand side of $(5.11)$ for $\boldsymbol{\mu}=(0, \mu)$ and $\mu \in P(n)$. By (4.31) and (5.9)

$$
f_{\mu}(a, v ; t ; p, q)=f_{\nu}\left(a q^{2 \mu_{n}}, v ; t ; p, q\right),
$$

where $\nu:=\left(\mu_{1}-\mu_{n}, \ldots, \mu_{n-1}-\mu_{n}, 0\right)$. For this special choice of $\boldsymbol{\mu}$, the same functional equation is satisfied by the right-side of (4.31) thanks to (2.14), leading to the following corollary.

Corollary 5.2. For $\mu \in P(n)$ and $a, b, v, p, q, t \in \mathbb{C}^{*}$ such that $a b=p q t^{2 n-3}$ and $|p|,|q|<1$,

$$
\begin{aligned}
& \left\langle R_{\mu}^{*}\left(z ; q / b t, q / a t ; q^{2}, t^{2} ; p^{2}\right)\right\rangle_{q / a t, p^{2} q / a t, q / b t, p^{2} q / b t, p t v, p t / v ; t^{2} ; p^{4}, q^{2}}^{(n)} \\
& \quad=\Delta_{\mu}^{0}\left(a^{2} t / p q \mid a^{2} t^{2-2 n}, a t v^{ \pm} ; q^{2}, t^{2} ; p^{2}\right) \frac{C_{\mu}^{-}\left(p q t ; q^{2}, t^{2} ; p^{2}\right) C_{\mu}^{+}\left(a^{2} ; q^{2}, t^{2} ; p^{2}\right)}{C_{2 \mu^{2}}^{0}\left((a p t)^{2} ; q^{2}, t^{2} ; p^{4}\right)} .
\end{aligned}
$$

According to [39, Corollary 4.14], if $a^{2} b^{2} u v t^{2 n-2}=p$ then $^{5}$

$$
\begin{aligned}
& \left\langle R_{\boldsymbol{\lambda}}^{*}(z ; a, b ; t ; p, q)\right\rangle_{a, a q, b, b q, u, v ; t ; p, q^{2}}^{(n)}=\Delta_{\boldsymbol{\lambda}}^{0}\left(a t^{n-1} / b \mid a^{2} q t^{n-1}, a u t^{n-1}, a v t^{n-1} ; t ; p, q\right) \\
& \quad \times \sum_{\boldsymbol{\mu}} \frac{\Delta_{\boldsymbol{\mu}}\left(1 / b^{2} \mid t^{n}, a^{2} q t^{n-1} ; t ; p, q^{2}\right)}{\Delta_{(1,2) \boldsymbol{\mu}}\left(1 / b^{2} \mid t^{n}, a^{2} q t^{n-1} ; t ; p, q\right)}\left\langle\begin{array}{c}
\boldsymbol{\lambda} \\
(1,2) \boldsymbol{\mu}
\end{array}\right\rangle_{\left[a t^{n-1} / b, a b t^{n-1}\right] ; t ; p, q} \cdot
\end{aligned}
$$

Combining this with (5.10) and using

$$
\frac{\Delta_{\boldsymbol{\mu}}^{0}\left(1 / b^{2} \mid t^{n}, a^{2} q t^{n-1} ; t ; p, q^{2}\right)}{\Delta_{(1,2) \boldsymbol{\mu}}^{0}\left(1 / b^{2} \mid t^{n}, a^{2} q t^{n-1} ; t ; p, q\right)}=\frac{1}{\Delta_{\boldsymbol{\mu}}^{0}\left(1 / b^{2} \mid q t^{n}, a^{2} q^{2} t^{n-1} ; p, q^{2}\right)},
$$

which is equal to 1 if $a^{2} b^{2} q t^{2 n-1}=p$, leads to the following quadratic summation formula.

Corollary 5.3. For $\boldsymbol{\lambda} \in P_{+}(n) \times P_{+}(n)$,

$$
\begin{gathered}
\sum_{\boldsymbol{\mu}} \frac{\Delta_{\boldsymbol{\mu}}\left(a \mid-; t^{2} ; p^{2}, q^{4}\right)}{\Delta_{(1,2) \boldsymbol{\mu}}\left(a \mid-; t^{2} ; p^{2}, q^{2}\right)}\left\langle\begin{array}{c}
\boldsymbol{\lambda} \\
(1,2) \boldsymbol{\mu}
\end{array}\right\rangle_{[a p q / t, p / q t] ; t^{2} ; p^{2}, q^{2}} \\
=\frac{C_{\boldsymbol{\lambda}}^{-}\left(p q t ; t^{2} ; p^{2}, q^{2}\right) C_{\boldsymbol{\lambda}}^{+}\left(a p^{2} / t^{2} ; t^{2} ; p^{2}, q^{2}\right)}{C_{(2,1) \boldsymbol{\lambda}^{2}}^{0}\left(a p^{2} q^{2} ; t^{2} ; p^{2}, q^{4}\right)}
\end{gathered}
$$

\subsection{The Kawanaka kernel}

For the Kawanaka kernel, $\mathcal{L}_{c}^{-}(x ; t ; p, q)$, we have a very similar definition and set of results as for the dual Littlewood kernel.

Let $x \in\left(\mathbb{C}^{*}\right)^{n}$ and $c, p, q, t, u, v \in \mathbb{C}^{*}$ such that $c^{2} u v=p q$ and $|p|,|q|<1$. Then the Kawanaka kernel is defined as [40]

$$
\begin{aligned}
\mathcal{L}_{c}^{-}(x ; t ; p, q):= & \prod_{i=1}^{n} \frac{1}{\Gamma_{p^{2}, q^{2}}\left(c u^{2} x_{i}^{ \pm}, c v^{2} x_{i}^{ \pm}\right)} \\
& \times \int \mathcal{K}_{c}\left(z^{2} ; x ; t^{2} ; p^{2}, q^{2}\right) \Delta_{\mathrm{S}}(z ; u, v ; t ; p, q) \frac{\mathrm{d} z_{1}}{z_{1}} \cdots \frac{\mathrm{d} z_{n}}{z_{n}},
\end{aligned}
$$

which once again does not depend on the individual choice of $u$ and $v$. The Kawanaka kernel satisfies the symmetry

$$
\mathcal{L}_{c}^{-}(x ; p q / t ; p, q)=\mathcal{L}_{c}^{-}(x ;-t ; p, q) \Gamma_{p^{2}, q^{2}}^{n}\left(t^{2}\right) \prod_{1 \leqslant i<j \leqslant n} \Gamma_{p^{2}, q^{2}}\left(t^{2} x_{i}^{ \pm} x_{j}^{ \pm}\right),
$$

\footnotetext{
${ }^{5}$ The statement of [39, Corollary 4.14] contains a minor typo in the argument of $\Delta_{\lambda}^{0}$.
} 
and factorisation formula

$$
\mathcal{L}_{(p q / t)^{1 / 2}}^{-}(x ;-t ; p, q)=\Gamma_{p^{2}, q^{2}}^{n}(p q / t) \prod_{i=1}^{n} \Gamma_{p, q}\left(-(p q / t)^{1 / 2} x_{i}^{ \pm}\right) \prod_{1 \leqslant i<j \leqslant n} \Gamma_{p^{2}, q^{2}}\left(p q x_{i}^{ \pm} x_{j}^{ \pm} / t\right) .
$$

Evaluating (5.14) at $x=a\langle\boldsymbol{\mu}\rangle_{n ; t^{2} ; p^{2}, q^{2}} / c$ and proceeded exactly as in the dual Littlewood case, also using

$$
\Gamma_{p^{2}, q^{2}}\left(z^{2}\right)=\Gamma_{p, q}(z,-z)
$$

we find

$$
\begin{aligned}
& \frac{\mathcal{L}_{c}^{-}\left(a\langle\boldsymbol{\mu}\rangle_{n ; t^{2} ; p^{2}, q^{2}} / c ; t ; p, q\right)}{\mathcal{L}_{c}^{-}\left(a\langle\mathbf{0}\rangle_{n ; t^{2} ; p^{2}, q^{2}} / c ; t ; p, q\right)} \\
& \quad=\left(u^{2} v^{2} t^{2 n-2}\right)^{2 \sum_{i=1}^{n} \mu_{i}^{(1)} \mu_{i}^{(2)}} \frac{\left\langle R_{\boldsymbol{\mu}}^{*}\left(z^{2} ; a, b ; q^{2}, t^{2} ; p^{2}\right)\right\rangle_{a^{1 / 2},-a^{1 / 2}, b^{1 / 2},-b^{1 / 2}, u, v ; t ; p, q}^{(n)}}{\Delta_{\boldsymbol{\mu}}^{0}\left(a t^{2 n-2} / b \mid a u^{2} t^{2 n-2}, a v^{2} t^{2 n-2} ; t^{2} ; p^{2}, q^{2}\right)},
\end{aligned}
$$

where $c^{2} u v=p q$ and $c^{2}=a b t^{2 n-2}$. Replacing $t \mapsto-t$ and then specialising $c=(p q / t)^{1 / 2}$, the left-hand side factors by (5.15), leading to our next theorem.

Theorem 5.4. For $\boldsymbol{\mu} \in P_{+}(n) \times P_{+}(n)$ and $a, b, u, v, p, q, t \in \mathbb{C}^{*}$ such that abt ${ }^{2 n-1}=p q$, uv $=t$ and $|p|,|q|<1$,

$$
\begin{aligned}
\left\langle R_{\boldsymbol{\mu}}^{*}\left(z^{2} ; a, b ; t^{2} ; p^{2}, q^{2}\right)\right\rangle_{a^{1 / 2},-a^{1 / 2}, b^{1 / 2},-b^{1 / 2}, u, v ;-t ; p, q}^{(n)} & \\
= & \Delta_{\boldsymbol{\mu}}^{0}\left(a t^{2 n-2} / b \mid a^{2} t^{2 n-2}, a u^{2} t^{2 n-2}, a v^{2} t^{2 n-2} ; t^{2} ; p^{2}, q^{2}\right) \\
& \times \frac{C_{\boldsymbol{\mu}}^{-}\left(p q t ; t^{2} ; p^{2}, q^{2}\right) C_{\boldsymbol{\mu}}^{+}\left(a^{2} t^{4 n-4} ; t^{2} ; p^{2}, q^{2}\right)}{C_{2 \boldsymbol{\mu}^{2}}^{0}\left(-a t^{2 n-1} ;-t ; p, q\right)} .
\end{aligned}
$$

From (2.13), (5.9) and (5.16) it follows that (5.17) for $\boldsymbol{\mu}=(0, \mu)$ again extends from partitions to weights.

Corollary 5.5. For $\mu \in P(n)$ and $a, b, u, v, p, q, t \in \mathbb{C}^{*}$ such that $a b t^{2 n-1}=p q$, $u v=t$ and $|p|,|q|<1$,

$$
\begin{aligned}
\left\langle R_{\mu}^{*}\left(z^{2} ; a, b ; q^{2}, t^{2} ; p^{2}\right)\right\rangle_{a^{1 / 2},-a^{1 / 2}, b^{1 / 2},-b^{1 / 2}, u, v ;-t ; p, q}^{(n)} & \\
= & \Delta_{\mu}^{0}\left(a t^{2 n-2} / b \mid a^{2} t^{2 n-2}, a u^{2} t^{2 n-2}, a v^{2} t^{2 n-2} ; q^{2}, t^{2} ; p^{2}\right) \\
& \times \frac{C_{\mu}^{-}\left(p q t ; q^{2}, t^{2} ; p^{2}\right) C_{\mu}^{+}\left(a^{2} t^{4 n-4} ; q^{2}, t^{2} ; p^{2}\right)}{C_{2 \mu^{2}}^{0}\left(-a t^{2 n-1} ; q,-t ; p\right)} .
\end{aligned}
$$

The analogue of (5.13) in the Kawanaka case is given by [39, Corollary 4.16]

$$
\begin{aligned}
\left\langle R_{\boldsymbol{\lambda}}^{*}\left(z^{2} ; a, b ; t^{2} ; p^{2}, q^{2}\right)\right\rangle_{a^{1 / 2},-a^{1 / 2}, b^{1 / 2},-b^{1 / 2}, u, v ;-t ; p, q}^{(n)} & \\
= & \Delta_{\boldsymbol{\lambda}}^{0}\left(a t^{2 n-2} / b \mid a^{2} t^{2 n-2}, a u^{2} t^{2 n-2}, a v^{2} t^{2 n-2} ; t^{2} ; p^{2}, q^{2}\right) \\
& \times \sum_{\boldsymbol{\mu}} \frac{\Delta_{\boldsymbol{\mu}}\left(1 / b \mid(-t)^{n},-a(-t)^{n-1} ;-t ; p, q\right)}{\Delta_{\boldsymbol{\mu}}\left(1 / b^{2} \mid t^{2 n}, a^{2} t^{2 n-2} ; t^{2} ; p^{2}, q^{2}\right)}\left\langle\begin{array}{c}
\boldsymbol{\lambda} \\
\boldsymbol{\mu}
\end{array}\right\rangle_{\left[a t^{2 n-2} / b, a b t^{2 n-2}\right] ; t^{2} ; p^{2}, q^{2}}
\end{aligned}
$$

for $a b u v t^{2 n-2}=p q$. Since for $a b t^{2 n-1}=p q$

$$
\frac{\Delta_{\boldsymbol{\mu}}^{0}\left(1 / b \mid(-t)^{n},-a(-t)^{n-1} ;-t ; p, q\right)}{\Delta_{\boldsymbol{\mu}}^{0}\left(1 / b^{2} \mid t^{2 n}, a^{2} t^{2 n-2} ; t^{2} ; p^{2}, q^{2}\right)}=\frac{1}{\Delta_{\mu}^{0}\left(1 / b \mid-(-t)^{n}, a(-t)^{n-1} ;-t ; p, q\right)}=1,
$$

we thus obtain the quadratic summation formula of the next corollary. 
Corollary 5.6. For $\boldsymbol{\lambda} \in P_{+}(n) \times P_{+}(n)$,

$$
\begin{aligned}
\sum_{\boldsymbol{\mu}} \frac{\Delta_{\boldsymbol{\mu}}(a \mid-;-t ; p, q)}{\Delta_{\boldsymbol{\mu}}\left(a^{2} \mid-; t^{2} ; p^{2}, q^{2}\right)}\left\langle\begin{array}{c}
\boldsymbol{\lambda} \\
\boldsymbol{\mu}
\end{array}\right\rangle_{\left[a^{2} p q / t, p q / t\right] ; t^{2} ; p^{2}, q^{2}} \\
=\frac{C_{\boldsymbol{\lambda}}^{-}\left(p q t ; t^{2} ; p^{2}, q^{2}\right) C_{\boldsymbol{\lambda}}^{+}\left(a^{2} p^{2} q^{2} / t^{2} ; t^{2} ; p^{2}, q^{2}\right)}{C_{2 \boldsymbol{\lambda}^{2}}^{0}(-a p q ;-t ; p, q)} .
\end{aligned}
$$

Here we note that $a$ in $(5.17)$ has been replaced by apqt $t^{1-2 n}$.

\section{Transition coefficients via elliptic hypergeometric integrals}

\subsection{Transition coefficients}

Recall that the Macdonald polynomials $P_{\lambda}$ indexed by weights $\lambda \in P(n)$ form a basis $\Lambda_{\mathrm{GL}(n)}$. In particular, any $\mathrm{BC}_{n}$-symmetric polynomial can be expanded in terms of Macdonald polynomials. If $\left\{f_{\lambda}\right\}$ (for $\lambda \in P_{+}(n)$ or $\lambda \in P(n)$ ) is a basis of $\Lambda_{\mathrm{BC}(n)}$ and $g$ an arbitrary element of $\Lambda_{\mathrm{BC}(n)}$ which expands in this basis as

$$
g=\sum_{\lambda} c_{\lambda} f_{\lambda}
$$

we will write $\left[f_{\lambda}\right] g$ to denote the coefficient $c_{\lambda}$. By (4.9) it then follows that

$$
\left[P_{\left(\lambda_{1}, \ldots, \lambda_{n}\right)}(q, t)\right] g=\left[P_{\left(-\lambda_{n}, \ldots,-\lambda_{1}\right)}(q, t)\right] g,
$$

so that it suffices to consider $\left[P_{\lambda}(q, t)\right] g$ for $\lambda \in P(n)$ such that $\lambda_{1} \geqslant 0$.

We are concerned with computing the transition coefficients

$$
c_{\lambda \mu}^{(n)}(q, t, s):=\left[P_{\lambda}(q, t)\right] \bar{P}_{\mu}^{*}(q, t, s) \in \mathbb{Q}(q, t)\left[s, s^{-1}\right]
$$

for $\lambda \in P(n)$ and $\mu \in P_{+}(n)$. Apart from

$$
c_{\left(\lambda_{1}, \ldots, \lambda_{n}\right), \mu}^{(n)}(q, t, s)=c_{\left(-\lambda_{n}, \ldots,-\lambda_{1}\right), \mu}^{(n)}(q, t, s),
$$

it follows from (4.13), the homogeneity of the Macdonald polynomials and (4.5) that $c_{\lambda \mu}^{(n)}$ satisfies the simple relations

$$
c_{\lambda \mu}^{(n)}(q, t, s)=c_{\lambda \mu}^{(n)}(1 / q, 1 / t, 1 / s)=(-1)^{|\lambda|-|\mu|} c_{\lambda \mu}^{(n)}(q, t,-s) .
$$

It will be convenient to scale $c_{\lambda \mu}^{(n)}(q, t, s)$ to a function that depends polynomially on $s^{2}$. To this end we define

$$
C_{\lambda \mu}^{(n)}(q, t, s):=\left(-s t^{n-1}\right)^{|\mu|-|\lambda|} q^{n\left(\mu^{\prime}\right)-n\left(\lambda^{\prime}\right)} t^{2 n(\lambda)-2 n(\mu)} c_{\lambda \mu}^{(n)}(q, t, s) \in \mathbb{Q}(q, t)\left[s^{2}\right],
$$

where we recall that $n(\lambda)$ and $n\left(\lambda^{\prime}\right)$ are defined for arbitrary weights $\lambda$ on page 7 . Some of the above symmetries for $c_{\lambda \mu}^{(n)}$ translate to

$$
C_{\lambda \mu}^{(n)}(q, t, s)=C_{\lambda \mu}^{(n)}(q, t,-s)=\left(\frac{q}{s^{2}}\right)^{|\lambda|} C_{\left(-\lambda_{n}, \ldots,-\lambda_{1}\right), \mu}^{(n)}(q, t, s) .
$$

Lemma 6.1. Let $\lambda, \mu \in P_{+}(n)$. Then

$$
C_{\lambda \mu}^{(n)}(q, t, s)=0 \quad \text { if } \lambda \not \subset \mu
$$

and $\mathcal{C}_{\lambda \lambda}^{(n)}(q, t, s)=1$. 
Proof. According to [35, Theorem 6.16], for $\lambda, \mu \in P_{+}(n)$,

$$
\bar{C}_{\lambda \mu}:=\left[P_{\lambda}\left(x^{ \pm} ; q, t\right)\right] \bar{P}_{\mu}^{*}(x ; q, t, s)=0
$$

if $\lambda \not \subset \mu$ (where $\bar{C}_{\lambda \mu} \in \mathbb{Q}(q, t)\left[s^{ \pm}\right]$), and $\bar{C}_{\mu \mu}=1$. We also have, for $\lambda \in P_{+}(n)$ and $\nu \in P(n)$, that

$$
\hat{C}_{\nu \lambda}:=\left[P_{\nu}(x ; q, t)\right] P_{\lambda}\left(x^{ \pm} ; q, t\right)=0
$$

if $|\lambda|-|\nu|$ is odd, or if there exists an $1 \leqslant i \leqslant n$ such that $\left|\nu_{i}\right|>\lambda_{i}$ (where $\hat{C}_{\nu \lambda} \in \mathbb{Q}(q, t)$ ), and $\hat{C}_{\lambda \lambda}=1$. Combining these two results the claim immediately follows.

Corollary 6.2. Let $N \in \mathbb{Z}$ and $\mu \in P_{+}(n)$. Then

$$
C_{\left(N^{n}\right), \mu}^{(n)}(q, t, s)=0 \quad \text { if }\left(|N|^{n}\right) \not \subset \mu .
$$

It seems to be a hard problem to get a handle on the general form of $c_{\lambda \mu}^{(n)}(q, t, s)$. When $n=1$ it is a straightforward exercise in basic hypergeometric series to show that for $N$ an integer and $k$ a nonnegative integer

$$
C_{(N),(k)}^{(1)}\left(q, t, s q^{1 / 2}\right)=\sum_{i=0}^{k} s^{2 i} q^{i(i+N)}\left[\begin{array}{l}
k \\
i
\end{array}\right]_{q}\left[\begin{array}{c}
k \\
i+N
\end{array}\right]_{q} .
$$

Here $\left[\begin{array}{l}k \\ i\end{array}\right]_{q}$ is the standard $q$-binomial coefficient

$$
\left[\begin{array}{c}
k \\
i
\end{array}\right]_{q}= \begin{cases}\frac{(q ; q)_{k}}{(q ; q)_{i}(q ; q)_{k-i}} & \text { for } 0 \leqslant i \leqslant k \\
0 & \text { otherwise }\end{cases}
$$

For $s=1$ the sum in (6.5) can be performed by the $q$-Chu-Vandermonde summation [13, equation (II.7)] to give

$$
C_{(N),(k)}^{(1)}\left(q, t, q^{1 / 2}\right)=\left[\begin{array}{c}
2 k \\
k+N
\end{array}\right]_{q}
$$

which generalises nicely for $\lambda=\left(N^{n}\right)$.

Theorem 6.3. For $N$ a nonnegative integer and $\mu \in P_{+}(n)$ such that $\left(N^{n}\right) \subset \mu$,

$$
C_{\left(N^{n}\right), \mu}^{(n)}\left(q, t, q^{1 / 2}\right)=\frac{C_{\mu}^{0}\left(t^{n} ; q, t\right) C_{\mu}^{+}\left(q t^{2 n-2} ; q, t\right)}{C_{\mu}^{-}(t ; q, t)} \prod_{i=1}^{n} \frac{\left(q t^{n-i} ; q\right)_{\mu_{i}}}{\left(q t^{n-i} ; q\right)_{\mu_{i}-N}\left(q t^{n-i} ; q\right)_{\mu_{i}+N}} .
$$

By (6.4) with $s^{2}=q$, the restriction that $N$ is nonnegative is non-essential, and it is of course clear that the right-hand side of (6.6) is invariant under negation of $N$. Replacing $\mu \mapsto \mu+\left(N^{n}\right)$ and using (2.13) and (4.14), it follows that (6.6) may also be stated as

$$
C_{\left(N^{n}\right), \mu+\left(N^{n}\right)}^{(n)}\left(q, t, q^{1 / 2}\right)=\frac{C_{\mu}^{0}\left(t^{n} ; q, t\right) C_{\mu}^{+}\left(q^{2 N+1} t^{2 n-2} ; q, t\right)}{C_{\mu}^{0}\left(q t^{n-1} ; q, t\right) C_{\mu}^{-}(t ; q, t)} .
$$

By the connection coefficient formula for $\bar{P}_{\mu}^{*}(q, t, s)$, see [35, Theorem 3.12], (6.6) leads to an expression for the more general transition coefficient $C_{\left(N^{n}\right), \mu}^{(n)}\left(q, t, s q^{1 / 2}\right)$. Since this result is not 
needed later, we omit the details. We do remark, however, that this expression does not (in an obvious manner) generalise (6.5), but instead extends the alternative form

$$
C_{(N),(k)}^{(n)}\left(q, t, s q^{1 / 2}\right)=\sum_{i=0}^{k} s^{i-N} \frac{(s q ; q)_{k}(s ; q)_{k-i}}{(s q ; q)_{i}}\left[\begin{array}{c}
k \\
i
\end{array}\right]_{q}\left[\begin{array}{c}
2 i \\
i+N
\end{array}\right]_{q},
$$

which obscures the fact that this is polynomial in $s^{2}$. The equality of (6.5) and (6.8) follows from the transformation formula

$$
{ }_{2} \phi_{1}\left[\begin{array}{c}
q^{-N} / b, q^{-N} \\
b q
\end{array} ; q, a^{2} q^{2 N+1}\right]=(a / b, a q ; q){ }_{N}{ }_{4} \phi_{3}\left[\begin{array}{c}
b q^{1 / 2},-b q^{1 / 2},-b q, q^{-N} \\
b^{2} q, a q, b q^{1-N} / a
\end{array} ; q, q\right],
$$

which we have not yet succeeded in generalising to the multivariable setting. The more general expression for $\mathcal{C}_{\left(N^{n}\right), \mu+\left(N^{n}\right)}^{(n)}\left(q, t, s q^{1 / 2}\right)$ does however show it to be a rational function in $\mathbb{Q}\left(q, t, s, t^{n}, q^{N} t^{n}\right)$.

\subsection{Proof of Theorem 6.3}

In this section we give a proof of Theorem 6.3 based on the elliptic hypergeometric integral (5.12).

Proposition 6.4. For $\mu \in P(n)$ and $q, t, s \in \mathbb{C}$ such that $0<|q|,|t|<1$ and $\left|s q^{\mu_{n}}\right|<1$,

$$
\begin{aligned}
\left\langle\bar{P}_{\mu}^{*}(z ; q, t, s)\right. & \left.\prod_{i=1}^{n} \frac{\theta\left(v z_{i} ; q\right)}{\left(s z_{i}^{ \pm} ; q\right)_{\infty}}\right\rangle_{q, t}^{(n)} \\
= & \left(-s t^{n-1}\right)^{-|\mu|} q^{-n\left(\mu^{\prime}\right)} t^{2 n(\mu)} \frac{C_{\mu}^{0}\left(t^{n}, s^{2} t^{n-1} ; q, t\right) C_{\mu}^{+}\left(s^{2} t^{2 n-2} ; q, t\right)}{C_{\mu}^{0}\left(s v t^{n-1}, s v^{-1} q t^{n-1} ; q, t\right) C_{\mu}^{-}(t ; q, t)} \\
& \times \prod_{i=1}^{n} \frac{\left(s v t^{i-1}, s v^{-1} q t^{i-1} ; q\right)_{\infty}}{\left(q t^{i-1}, s^{2} t^{i-1} ; q\right)_{\infty}} .
\end{aligned}
$$

The integrand on the left has simple poles at $z_{i}=\left(s q^{\mu_{n}+k}\right)^{\sigma}$ for $\sigma \in\{-1,1\}, 1 \leqslant i \leqslant n$ and $k$ a nonnegative integer. The condition $\left|s q^{\mu_{n}}\right|<1$ ensures that the poles at $z_{i}=s q^{\mu_{n}+k}$ lie in the interior of $\mathbb{T}^{n}$ and the poles at $z_{i}=s^{-1} q^{-\mu_{n}-k}$ lie in the exterior.

Before showing how (6.9) follows from (5.12), we first use the former to prove Theorem 6.3.

Proof of Theorem 6.3. Let $N$ be a nonnegative integer and replace $s, v \mapsto q^{N+1 / 2}$ in (6.9). By (2.8) and (4.14) this yields

$$
\begin{gathered}
\left(-q^{N+1 / 2} t^{n-1}\right)^{|\mu|} q^{n\left(\mu^{\prime}\right)} t^{-2 n(\mu)}\left\langle\bar{P}_{\mu+\left(N^{n}\right)}^{*}\left(z ; q, t, q^{1 / 2}\right) \prod_{i=1}^{n} z_{i}^{-N}\right\rangle_{q, t}^{(n)} \\
=\frac{C_{\mu}^{0}\left(t^{n} ; q, t\right) C_{\mu}^{+}\left(q^{2 N+1} t^{2 n-2} ; q, t\right)}{C_{\mu}^{0}\left(q t^{n-1} ; q, t\right) C_{\mu}^{-}(t ; q, t)}
\end{gathered}
$$

By the orthogonality (4.3),

$$
c_{\lambda \mu}(q, t, s)=\frac{\left\langle P_{\lambda}\left(z^{-1} ; q, t\right) \bar{P}_{\mu}^{*}(z ; q, t, s)\right\rangle_{q, t}^{(n)}}{\left\langle P_{\lambda}\left(z^{-1} ; q, t\right) P_{\lambda}(z ; q, t)\right\rangle_{q, t}^{(n)}} .
$$

Since $P_{\left(N^{n}\right)}(z ; q, t)=\prod_{i=1}^{n} z_{i}^{N}$, for $\lambda=\left(N^{n}\right)$ this simplifies to

$$
\mathcal{c}_{\left(N^{n}\right), \mu}(q, t, s)=\left\langle\bar{P}_{\mu}^{*}(z ; q, t, s) \prod_{i=1}^{n} z_{i}^{-N}\right\rangle_{q, t}^{(n)} .
$$


Shifting $\mu \mapsto \mu+\left(N^{n}\right)$ and using (6.3), this yields

$$
\mathcal{C}_{\left(N^{n}\right), \mu+\left(N^{n}\right)}\left(q, t, q^{1 / 2}\right)=\frac{C_{\mu}^{0}\left(t^{n} ; q, t\right) C_{\mu}^{+}\left(q^{2 N+1} t^{2 n-2} ; q, t\right)}{C_{\mu}^{0}\left(q t^{n-1} ; q, t\right) C_{\mu}^{-}(t ; q, t)} .
$$

Equating this with (6.10) we obtain (6.7).

Proof of Proposition 6.4. We begin by making the substitutions $a \mapsto s t^{2 n-2}$ and $v \mapsto v / p$ in (5.12), resulting in

$$
\begin{aligned}
\left\langle R_{\mu}^{*}\left(z ; s / p, q t^{1-2 n} / s ; q^{2}, t^{2} ; p^{2}\right)\right\rangle_{q t^{1-2 n} / s, p^{2} q t^{1-2 n} / s, s / p, s p, t v, p^{2} t / v ; t^{2} ; p^{4}, q^{2}}^{(n)} & \\
= & \Delta_{\mu}^{0}\left(s^{2} t^{4 n-3} / p q \mid s^{2} t^{2 n-2}, s t^{2 n-1} v / p, s p t^{2 n-1} / v ; q^{2}, t^{2} ; p^{2}\right) \\
& \times \frac{C_{\mu}^{+}\left(s^{2} t^{4 n-4} ; q^{2}, t^{2} ; p^{2}\right) C_{\mu}^{-}\left(p q t ; q^{2}, t^{2} ; p^{2}\right)}{C_{2 \mu^{2}}^{0}\left(s p^{2} t^{4 n-2} ; q^{2}, t^{2} ; p^{4}\right)} .
\end{aligned}
$$

The general method for taking the $p \rightarrow 0$ limit of integrals such as (6.11) was developed in [37] and has also applied more recently in [1]. It relies on a trick to break the $\mathrm{BC}_{n}$ symmetry, resulting in an integral with $S_{n}$-symmetry only. Denote the left-hand side of (6.11) by $\mathscr{L}$. Then, by

$$
\Gamma_{p^{2}, q}(z, p z)=\Gamma_{p, q}(z)
$$

we have

$$
\begin{aligned}
\mathscr{L}= & \frac{1}{S_{n}\left(q t^{1-2 n} / s, p^{2} q t^{1-2 n} / s, s / p, s p, t v, p^{2} t / v ; t^{2} ; p^{4}, q^{2}\right)} \\
& \times \int R_{\mu}^{*}\left(z ; s / p, q t^{1-2 n} / s ; q^{2}, t^{2} ; p^{2}\right) \Delta_{\mathrm{S}}\left(z ; t v, p^{2} t / v ; t^{2} ; p^{4}, q^{2}\right) \\
& \times \prod_{i=1}^{n} \Gamma_{p^{2}, q^{2}}\left(s z_{i}^{ \pm} / p, q t^{-n} z_{i}^{ \pm} / s\right) \frac{\mathrm{d} z_{1}}{z_{1}} \cdots \frac{\mathrm{d} z_{n}}{z_{n}} .
\end{aligned}
$$

By $\mathrm{BC}_{n}$-symmetry and the identity

$$
1=\sum_{\sigma \in\{ \pm 1\}^{n}} \prod_{i=1}^{n} \frac{\theta\left(t_{0} z_{i}^{\sigma_{i}}, t_{1} z_{i}^{\sigma_{i}}, t_{2} z_{i}^{\sigma_{i}}, t^{n-1} t_{0} t_{1} t_{2} z_{i}^{-\sigma_{i}} ; q\right)}{\theta\left(z_{i}^{2 \sigma_{i}}, t^{i-1} t_{0} t_{1}, t^{i-1} t_{0} t_{2}, t^{i-1} t_{1} t_{2} ; q\right)} \prod_{1 \leqslant i<j \leqslant n} \frac{\theta\left(t z_{i}^{\sigma_{i}} z_{j}^{\sigma_{j}} ; q\right)}{\theta\left(z_{i}^{\sigma_{i}} z_{j}^{\sigma_{j}} ; q\right)}
$$

we may multiply the integrand by the symmetry-breaking factor

$$
2^{n} \prod_{i=1}^{n} \frac{\theta\left(s z_{i} / p, q t^{1-2 n} z_{i} / s, t v z_{i}, q v z_{i}^{-1} / p ; q^{2}\right)}{\theta\left(z_{i}^{2}, q t^{2 i-2 n-1} / p, s t^{2 i-1} v / p, q t^{2 i-2 n} v / s ; q^{2}\right)} \prod_{1 \leqslant i<j \leqslant n} \frac{\theta\left(t^{2} z_{i} z_{j} ; q^{2}\right)}{\theta\left(z_{i} z_{j} ; q^{2}\right)} .
$$

By the functional equation (2.5) for the elliptic gamma function, this leads to

$$
\begin{aligned}
\mathscr{L}= & \frac{2^{n} \kappa_{n}\left(p^{4}, q^{2}\right) \Gamma_{p^{4}, q^{2}}^{n}\left(t^{2}\right)}{S_{n}\left(q t^{1-2 n} / s, p^{2} q t^{1-2 n} / s, s p, s / p, t v, p^{2} t / v ; t^{2} ; p^{4}, q^{2}\right)} \\
& \times \int R_{\mu}^{*}\left(z ; s / p, q t^{1-2 n} / s ; q^{2}, t^{2} ; p^{2}\right) \prod_{i=1}^{n} \theta\left(p q z_{i} / v ; q^{2}\right) \\
& \times \prod_{i=1}^{n} \frac{\Gamma_{p^{2}, q^{2}}\left(s\left(p z_{i}\right)^{ \pm}, p q t^{1-2 n}\left(p z_{i}\right)^{ \pm} / s\right) \Gamma_{p^{4}, q^{2}}\left(p t v\left(p z_{i}\right)^{ \pm}, p^{2} t z_{i}^{ \pm} / v\right)}{\Gamma_{p^{4}, q^{2}}\left(p^{2}\left(p^{2} z_{i}^{2}\right)^{ \pm}\right)} \\
& \times \prod_{1 \leqslant i<j \leqslant n} \frac{\Gamma_{p^{4}, q^{2}}\left(p^{2} t^{2}\left(p^{2} z_{i} z_{j}\right)^{ \pm}, t^{2}\left(z_{i} / z_{j}\right)^{ \pm}\right)}{\Gamma_{p^{4}, q^{2}}\left(p^{2}\left(p^{2} z_{i} z_{j}\right)^{ \pm},\left(z_{i} / z_{j}\right)^{ \pm}\right)} \frac{\mathrm{d} z_{1}}{z_{1}} \cdots \frac{\mathrm{d} z_{n}}{z_{n}} .
\end{aligned}
$$


We can scale the contour $C$ by a factor $1 / p$ without crossing any poles. Then replacing $z_{i} \mapsto z_{i} / p$ (so that the contour is once again given by $C$ ) and using (4.37) with

$$
(x, a, b, p, q, t) \mapsto\left(z / p, s / p, q t^{1-2 n} / s, p^{2}, q^{2}, t^{2}\right),
$$

this yields

$$
\begin{aligned}
\mathscr{L}= & \frac{2^{n} q^{4 n\left(\mu^{\prime}\right)} t^{-4 n(\mu)}\left(s^{2} q t^{4 n-3} / p\right)^{|\mu|} \kappa_{n}\left(p^{4}, q^{2}\right) \Gamma_{p^{4}, q^{2}}^{n}\left(t^{2}\right)}{S_{n}\left(q t^{1-2 n} / s, p^{2} q t^{1-2 n} / s, s p, s / p, t v, p^{2} t / v ; t^{2} ; p^{4}, q^{2}\right)} \\
& \times \int R_{\mu}^{*}\left(z ; s, p q t^{1-2 n} / s ; q^{2}, t^{2} ; p^{2}\right) \prod_{i=1}^{n} \theta\left(q z_{i} / v ; q^{2}\right) \\
& \times \prod_{i=1}^{n} \frac{\Gamma_{p^{2}, q^{2}}\left(s z_{i}^{ \pm}, p q t^{1-2 n} z_{i}^{ \pm} / s\right) \Gamma_{p^{4}, q^{2}}\left(p t v z_{i}^{ \pm}, p t z_{i} / v, p^{3} t z_{i}^{-1} / v\right)}{\Gamma_{p^{4}, q^{2}}\left(p^{2} z_{i}^{ \pm 2}\right)} \\
& \times \prod_{1 \leqslant i<j \leqslant n} \frac{\Gamma_{p^{4}, q^{2}}\left(p^{2} t^{2}\left(z_{i} z_{j}\right)^{ \pm}, t^{2}\left(z_{i} / z_{j}\right)^{ \pm}\right)}{\Gamma_{p^{4}, q^{2}}\left(p^{2}\left(z_{i} z_{j}\right)^{ \pm},\left(z_{i} / z_{j}\right)^{ \pm}\right)} \frac{\mathrm{d} z_{1}}{z_{1}} \cdots \frac{\mathrm{d} z_{n}}{z_{n}} .
\end{aligned}
$$

Taking the $p \rightarrow 0$ limit using (4.33) and

$$
\lim _{p \rightarrow 0} \Gamma\left(p^{\alpha} z ; p, q\right)= \begin{cases}1 /(z ; q)_{\infty} & \text { if } \alpha=0 \\ 1 & \text { if } 0<\alpha<1 \\ (q / z ; q)_{\infty} & \text { if } \alpha=1\end{cases}
$$

we obtain

$$
\begin{aligned}
\lim _{p \rightarrow 0}\left(\frac{p}{q t}\right)^{|\mu|} \mathscr{L}= & \left(-s t^{2 n-2}\right)^{3|\mu|} q^{6 n\left(\mu^{\prime}\right)} t^{-8 n(\mu)} \prod_{i=1}^{n} \frac{\left(q^{2} t^{2 i-2}, s^{2} t^{2 i-2} ; q^{2}\right)_{\infty}}{\left(s q t^{2 i-2} v^{ \pm} ; q^{2}\right)_{\infty}} \\
& \times \frac{C_{\mu}^{-}\left(t^{2} ; q^{2}, t^{2}\right)}{C_{\mu}^{0}\left(t^{2 n} ; q^{2}, t^{2}\right)}\left\langle\bar{P}_{\mu}^{*}\left(z ; q^{2}, t^{2}, s^{2}\right) \prod_{i=1}^{n} \frac{\theta\left(q z_{i} / v ; q^{2}\right)}{\left(s z_{i}^{ \pm} ; q^{2}\right)_{\infty}}\right\rangle_{q^{2}, t^{2}}^{(n)}
\end{aligned}
$$

where we have also used the evaluation (4.2).

Taking the same limit in the right-hand side of (6.11) yields

$$
\lim _{p \rightarrow 0}\left(\frac{p}{q t}\right)^{|\mu|} \mathscr{R}=\left(s t^{2 n-2}\right)^{2|\mu|} q^{4 n\left(\mu^{\prime}\right)} t^{-4 n(\mu)} \frac{C_{\mu}^{0}\left(s^{2} t^{2 n-2} ; q^{2}, t^{2}\right) C_{\mu}^{+}\left(s^{2} t^{4 n-4} ; q^{2}, t^{2}\right)}{C_{\mu}^{0}\left(s q t^{2 n-2} v^{ \pm} ; q^{2}, t^{2}\right)} .
$$

Equating the right-hand sides of (6.12) and (6.13), and replacing $v \mapsto q / v$ results in (6.9) with $(q, t) \mapsto\left(q^{2}, t^{2}\right)$, completing the proof.

\section{The elliptic hypergeometric function $\Phi_{\lambda}$}

In this section we define a new elliptic hypergeometric function,

$$
\Phi_{\lambda}=\Phi_{\lambda}(q, t ; p)=\Phi_{\lambda}(a ; b, c, d ; q, t ; p)
$$

study its symmetries and prove two summation formulas for one-parameter specialisations of $\{a, b, c, d\}$. The $p \rightarrow 0$ limit of $\Phi_{\lambda}(q, t ; p)$ will play an important role in proving the $q$, $t$-branching rules (1.8) and (1.12). 
For $\lambda$ a partition and $a, b, c, d, p, q, t \in \mathbb{C}^{*}$ such that $|p|<1$, the elliptic hypergeometric function $\Phi_{\lambda}(q, t ; p)$ is defined as

$$
\begin{gathered}
\Phi_{\lambda}(a ; b, c, d ; q, t ; p) \\
:=\frac{1}{\Delta_{\lambda}^{0}\left(e \mid f ; q^{2}, t^{2} ; p^{2}\right)} \sum_{\mu \subset \lambda} \frac{C_{\mu}^{-}\left(p q t ; q^{2}, t^{2} ; p^{2}\right) C_{\mu}^{+}\left(a^{2} p^{2} / q^{2} ; q^{2}, t^{2} ; p^{2}\right)}{C_{\mu}^{0}\left(a p, b p, c p, d p ; q^{2}, t^{2} ; p\right)}\left\langle\begin{array}{l}
\lambda \\
\mu
\end{array}\right\rangle_{[e, f] ; q^{2}, t^{2} ; p^{2}} \\
=\frac{1}{\Delta_{\lambda}^{0}\left(e \mid f ; q^{2}, t^{2} ; p^{2}\right)} \sum_{\mu \subset \lambda}\left(\left(-e q^{2}\right)^{|\mu|} q^{2 n\left(\mu^{\prime}\right)} t^{-2 n(\mu)}\right. \\
\left.\quad \times \frac{C_{\mu}^{-}\left(p q t ; q^{2}, t^{2} ; p^{2}\right) C_{\mu}^{+}\left(a^{2} / q^{2} ; q^{2}, t^{2} ; p^{2}\right)}{C_{\mu}^{0}\left(a, b, c, d ; q^{2}, t^{2} ; p\right)}\left\langle\begin{array}{l}
\lambda \\
\mu
\end{array}\right\rangle_{[e, f] ; q^{2}, t^{2} ; p^{2}}\right),
\end{gathered}
$$

where $e:=b c d / a q^{2}$ and $f:=b c d q / a^{3} p t$. The equality of the two expressions on the right of (7.1) is a direct consequence of the quasi-periodicity (2.9) of the elliptic $C$-symbols. We also note that $\Phi_{\lambda}(a ;-a, c, d ; q, t ; p)$ is a function of $a^{2}$ only, so that $\Phi_{\lambda}(a ;-a, c, d ; q, t ; p)=\Phi_{\lambda}(-a ; a, c, d ; q, t ; p)$.

For later use we also define the following basic hypergeometric analogue of $\Phi_{\lambda}(q, t ; p)$ :

$$
\Phi_{\lambda}(q, t)=\Phi_{\lambda}(a ; b, c, d ; q, t):=\sum_{\mu \subset \lambda}(-1)^{|\mu|} q^{-n\left(\mu^{\prime}\right)} t^{n(\mu)} \frac{C_{\mu}^{+}\left(a^{2} / q, s^{2} ; q, t\right)}{C_{\mu}^{0}(a, b, c, d ; q, t)}\left[\begin{array}{l}
\lambda \\
\mu
\end{array}\right]_{q, t, s},
$$

where $s^{2}:=b c d / a q$. The reason for renaming $e$ in (7.1) as $s^{2}$ is the convention of writing $\left[\begin{array}{l}\lambda \\ \mu\end{array}\right]_{q, t, s}$. Here we recall that the $q, t, s$-binomial coefficient is a function of $s^{2}$ only. For $\lambda$ a partition of length at most one $\Phi_{\lambda}(q, t)$ is independent of $t$ and simplifies to a balanced, terminating ${ }_{5} \phi_{4}$ basic hypergeometric series [13]:

$$
\Phi_{(N)}(a ; b, c, d ; q, t)={ }_{5} \phi_{4}\left[\begin{array}{c}
a q^{-1 / 2},-a q^{-1 / 2},-a, b c d q^{N-1} / a, q^{-N} \\
a^{2} q^{-1}, b, c, d
\end{array} ; q, q\right] .
$$

Lemma 7.1. We have

$$
\lim _{p \rightarrow 0} \Phi_{\lambda}(a ; b, c, d ; q, t ; p)=\Phi_{\lambda}\left(a ; b, c, d ; q^{2}, t^{2}\right) .
$$

Proof. By (2.9a),

$$
\Delta_{\lambda}^{0}(e \mid f ; q, t ; p)=(e q)^{|\lambda|} q^{2 n(\lambda)} t^{-2 n(\lambda)} \Delta_{\lambda}^{0}(e \mid f p ; q, t ; p) .
$$

Replacing $(p, q, t) \mapsto\left(p^{2}, q^{2}, t^{2}\right)$ and substituting the above in the second form for $\Phi$, the $p \rightarrow 0$ limit can be taken using (4.44) and $\lim _{p \rightarrow 0} C_{\lambda}^{0, \pm}\left(z p ; q^{2}, t^{2} ; p^{2}\right)=1$, resulting in the claim.

The function $\Phi_{\lambda}$ satisfies the following symmetries.

Lemma 7.2. For $\lambda$ a partition

$$
\Phi_{\lambda}(1 / a ; 1 / b, 1 / c, 1 / d ; 1 / q, 1 / t ; p)=\Phi_{\lambda}(a ; b, c, d ; q, t ; p)
$$

and

$$
\Phi_{\lambda^{\prime}}(a ; b, c, d ; t, q ; p)=\Phi_{\lambda}(1 / a ; 1 / b, 1 / c, 1 / d ; q, t ; p) .
$$

Combining these two results further yields

$$
\Phi_{\lambda}(a ; b, c, d ; 1 / q, 1 / t ; p)=\Phi_{\lambda^{\prime}}(a ; b, c, d ; t, q ; p) .
$$


Proof. Throughout the proof $e$ and $f$ are fixed as $e=b c d / a q^{2}$ and $f=b c d q / a^{3} p t$.

By (2.11) and (4.43),

$$
\begin{aligned}
\Phi_{\lambda}(1 / a ; 1 / b, 1 / c, 1 / d ; 1 / q, 1 / t ; p) & \frac{1}{\Delta_{\lambda}^{0}\left(1 / e \mid 1 / f p^{2} ; 1 / q^{2}, 1 / t^{2} ; p^{2}\right)} \sum_{\mu \subset \lambda}\left(\left(-e q^{2}\right)^{-|\mu|} q^{-2 n\left(\mu^{\prime}\right)} t^{2 n(\mu)}\right. \\
& \left.\times \frac{C_{\mu}^{-}\left(p / q t ; 1 / q^{2}, 1 / t^{2} ; p^{2}\right) C_{\mu}^{+}\left(q^{2} / a^{2} ; 1 / q^{2}, 1 / t^{2} ; p^{2}\right)}{C_{\mu}^{0}\left(1 / a, 1 / b, 1 / c, 1 / d ; 1 / q^{2}, 1 / t^{2} ; p\right)}\left\langle\begin{array}{l}
\lambda \\
\mu
\end{array}\right\rangle_{\left[1 / e, 1 / f p^{2}\right] ; 1 / q^{2}, 1 / t^{2} ; p^{2}}\right) \\
= & \left(\frac{f^{2} p^{6}}{e^{3} q^{6}}\right)^{|\lambda|} \frac{q^{-8 n\left(\lambda^{\prime}\right)} t^{8 n(\lambda)}}{\Delta_{\lambda}^{0}\left(e q^{2} / p^{4} \mid f p^{2} ; q^{2}, t^{2} ; p^{2}\right)} \\
& \times \sum_{\mu \subset \lambda}(e p q / t)^{|\mu|} t^{-4 n(\mu)} \frac{C_{\mu}^{-}\left(q t / p ; q^{2}, t^{2} ; p^{2}\right) C_{\mu}^{+}\left(a^{2} / q^{2} ; q^{2}, t^{2} ; p^{2}\right)}{C_{\mu}^{0}\left(a, b, c, d ; q^{2}, t^{2} ; p\right)}\left\langle\begin{array}{l}
\lambda\rangle_{\mu} \\
\rangle_{[e, f] ; q^{2}, t^{2} ; p^{2}}
\end{array} .\right.
\end{aligned}
$$

The first claim now follows by applying (2.9a) to $\Delta_{\lambda}^{0}$ and (2.9b) to $C_{\mu}^{-}$.

Similarly, by (2.10) and (4.43),

$$
\begin{aligned}
\Phi_{\lambda^{\prime}}(a ; b, c, d ; t, q ; p) & \frac{1}{\Delta_{\lambda^{\prime}}^{0}\left(e q^{2} / t^{2} \mid f t^{2} / q^{2} ; t^{2}, q^{2} ; p^{2}\right)} \sum_{\mu \subset \lambda}\left(\left(-e q^{2}\right)^{|\mu|} q^{-2 n\left(\mu^{\prime}\right)} t^{2 n(\mu)}\right. \\
& \left.\times \frac{C_{\mu^{\prime}}^{-}\left(p q t ; t^{2}, q^{2} ; p^{2}\right) C_{\mu^{\prime}}^{+}\left(a^{2} / t^{2} ; t^{2}, q^{2} ; p^{2}\right)}{C_{\mu^{\prime}}^{0}\left(a, b, c, d ; t^{2}, q^{2} ; p\right)}\left\langle\begin{array}{l}
\lambda^{\prime} \\
\mu^{\prime}
\end{array}\right\rangle_{\left[e q^{2} / t^{2}, f t^{2} / q^{2}\right] ; t^{2}, q^{2} ; p^{2}}\right) \\
= & \left(\frac{e^{3} p^{2} q^{10}}{f^{2} t^{4}}\right)^{|\lambda|} \frac{q^{-8 n\left(\lambda^{\prime}\right)} t^{8 n(\lambda)}}{\Delta_{\lambda}^{0}\left(1 / e p^{4} q^{4} \mid q^{2} / f t^{2} ; q^{2}, t^{2} ; p^{2}\right)} \sum_{\mu \subset \lambda}\left(\left(-e q^{2}\right)^{-|\mu|} q^{2 n\left(\mu^{\prime}\right)} t^{-2 n(\mu)}\right. \\
& \left.\times \frac{C_{\mu}^{-}\left(p q t ; q^{2}, t^{2} ; p^{2}\right) C_{\mu^{\prime}}^{+}\left(1 / a^{2} q^{2} ; q^{2}, t^{2} ; p^{2}\right)}{C_{\mu^{\prime}}^{0}\left(1 / a, 1 / b, 1 / c, 1 / d ; q^{2}, t^{2} ; p\right)}\left\langle\begin{array}{l}
\lambda \\
\mu
\end{array}\right\rangle_{\left[1 / e q^{4}, q^{2} / f p^{2} t^{2}\right] ; q^{2}, t^{2} ; p^{2}}\right) .
\end{aligned}
$$

By (2.9a) applied to $\Delta_{\lambda}^{0}$ this yields

$$
\begin{aligned}
\Phi_{\lambda^{\prime}}(a ; b, c, d ; t, q ; p) & \\
= & \frac{1}{\Delta_{\lambda}^{0}\left(1 / e q^{4} \mid q^{2} / f p^{2} t^{2} ; q^{2}, t^{2} ; p^{2}\right)} \sum_{\mu \subset \lambda}\left(\left(-e q^{2}\right)^{-|\mu|} q^{2 n\left(\mu^{\prime}\right)} t^{-2 n(\mu)}\right. \\
& \left.\times \frac{C_{\mu}^{-}\left(p q t ; q^{2}, t^{2} ; p^{2}\right) C_{\mu^{\prime}}^{+}\left(1 / a^{2} q^{2} ; q^{2}, t^{2} ; p^{2}\right)}{C_{\mu^{\prime}}^{0}\left(1 / a, 1 / b, 1 / c, 1 / d ; q^{2}, t^{2} ; p\right)}\left\langle\begin{array}{l}
\lambda \\
\mu
\end{array}\right\rangle_{\left[1 / e q^{4}, q^{2} / f p^{2} t^{2}\right] ; q^{2}, t^{2} ; p^{2}}\right) \\
= & \Phi_{\lambda}(1 / a ; 1 / b, 1 / c, 1 / d ; q, t ; p) .
\end{aligned}
$$

For the final equality we note that if for $e=e(a, b, c, d ; q)$ and $f=f(a, b, c, d ; q, t ; p)$ we define $\hat{e}:=e(1 / a, 1 / b, 1 / c, 1 / d ; q)$ and $\hat{f}:=f(1 / a, 1 / b, 1 / c, 1 / d ; q, t ; p)$, then $e q^{2}=1 / \hat{e} q^{2}$ (so that $\left.1 / e q^{4}=\hat{e}\right)$ and $q^{2} / f p^{2} t^{2}=\hat{f}$.

The elliptic hypergeometric series (7.1) is balanced but not, generally, (very-)well-poised. For example, as follows from (4.40), the one-row case of $\Phi_{\lambda}$ is given by

$$
\begin{aligned}
\Phi_{(N)}(a ; b, c, d ; q, t ; p)=\sum_{k=0}^{N} & \left(\frac{\left(e p^{2} q^{2} / f ; q^{2}, p^{2}\right)_{2 k}}{\left(e / f ; q^{2}, p^{2}\right)_{2 k}} \frac{\left(a^{2} p^{2}, a^{2} p^{2} / q^{2} ; q^{4}, p^{2}\right)_{k}}{\left(a p, b p, c p, d p ; q^{2}, p\right)_{k}}\right. \\
& \left.\times \frac{\left(e / f, p q t, e q^{2 N}, q^{-2 N} ; q^{2}, p^{2}\right)_{k}}{\left(p^{2} q^{2}, a^{2} p^{2} / q^{2}, p^{2} q^{2-2 N} / f, e p^{2} q^{2 N+2} / f ; q^{2}, p^{2}\right)_{k}}\right),
\end{aligned}
$$


generalising (7.3). Since

$$
\frac{\left(a^{2} p^{2}, a^{2} p^{2} / q^{2} ; q^{4}, p^{2}\right)_{k}}{\left(a p, b p, c p, d p ; q^{2}, p\right)_{k}}=\frac{\left(-a p,-a p^{2}, \pm a p / q, \pm a p^{2} / q ; q^{2}, p^{2}\right)_{k}}{\left(b p, b p^{2}, c p, c p^{2}, d p, d p^{2} ; q^{2}, p^{2}\right)_{k}},
$$

$\Phi_{(N)}(a ; b, c, d ; q, t ; p)$ is a balanced elliptic hypergeometric series of the form (2.17) for $r=9$. The ratio of elliptic shifted factorials (7.6) is however not well-poised, and there are exactly eight choices for $b, c, d$ for which it is: $b \in\{-a,-a t / q\}$ and $c, d$ one of

$$
\begin{aligned}
& c=-d=a t, \\
& c=-d=a / q, \\
& c=\sigma a t, \quad d=-\sigma a / q,
\end{aligned}
$$

where $\sigma \in\{-1,1\}$. By (7.4b) only four of these are independent, and irrespective of the choice of $b,(7.7 \mathrm{a})$ and $(7.7 \mathrm{~b})$ as well as the two choices for $\sigma$ in $(7.7 \mathrm{c})$ are related by conjugation.

For two of these independent choices we have a closed-form evaluation, generalising $(2.19 \mathrm{~b})$ and (2.19d). Our first result is a generalisation of (2.19b), which is recovered for $\lambda=(N)$ after making the substitution $(a, t) \mapsto\left(a q^{4} / b p^{2}, b p / q\right)$ followed by $\left(p^{2}, q^{2}\right) \mapsto(p, q)$.

Theorem 7.3. For $\lambda$ a partition,

$$
\Phi_{\lambda}\left(a^{1 / 2} ;-a^{1 / 2}, a^{1 / 2} t,-a^{1 / 2} t ; q, t ; p\right)= \begin{cases}\frac{\Delta_{\mu}\left(a t^{2} / q^{2} \mid-; q^{4}, t^{2} ; p^{2}\right)}{\Delta_{2 \mu}\left(a t^{2} / q^{2} \mid q t / p ; q^{2}, t^{2} ; p^{2}\right)} & \text { if } \lambda=2 \mu, \\ 0 & \text { otherwise. }\end{cases}
$$

Proof. By the inversion relation (4.42), the quadratic summation of Corollary 5.3 implies

$$
\begin{aligned}
\sum_{\boldsymbol{\mu}} \frac{C_{\boldsymbol{\mu}}^{-}\left(p q t ; t^{2} ; p^{2}, q^{2}\right) C_{\boldsymbol{\mu}}^{+}\left(a p^{2} / t^{2} ; t^{2} ; p^{2}, q^{2}\right)}{C_{(2,1) \boldsymbol{\mu}^{2}}^{0}\left(a p^{2} q^{2} ; t^{2} ; p^{2}, q^{4}\right)}\left\langle\begin{array}{l}
\boldsymbol{\lambda} \\
\boldsymbol{\mu}
\end{array}\right\rangle_{[a, q t / p] ; t^{2} ; p^{2}, q^{2}} \\
\quad= \begin{cases}\frac{\Delta_{\boldsymbol{\nu}}\left(a \mid-; t^{2} ; p^{2}, q^{4}\right)}{\Delta_{(1,2) \boldsymbol{\nu}}\left(a \mid-; t^{2} ; p^{2}, q^{2}\right)} & \text { if } \boldsymbol{\lambda}=(1,2) \boldsymbol{\nu}, \\
0 & \text { otherwise. }\end{cases}
\end{aligned}
$$

Choosing $\boldsymbol{\lambda}=(0, \lambda)$ and noting that

$$
C_{\mu^{2}}^{0}\left(a p^{2} q^{2} ; q^{4}, t^{2} ; p^{2}\right)=C_{\mu}^{0}\left( \pm a^{1 / 2} p q, \pm a^{1 / 2} p q / t ; q^{2}, t^{2} ; p\right),
$$

leads to

$$
\begin{aligned}
& \sum_{\mu} \frac{C_{\mu}^{-}\left(p q t ; q^{2}, t^{2} ; p^{2}\right) C_{\mu}^{+}\left(a p^{2} / t^{2} ; q^{2}, t^{2} ; p^{2}\right)}{C_{\mu}^{0}\left( \pm a^{1 / 2} p q, \pm a^{1 / 2} p q / t ; q^{2}, t^{2} ; p\right)}\left\langle\begin{array}{l}
\lambda \\
\mu
\end{array}\right\rangle_{[a, q t / p] ; q^{2}, t^{2} ; p^{2}} \\
& \quad= \begin{cases}\frac{\Delta_{\nu}\left(a \mid-; q^{4}, t^{2} ; p^{2}\right)}{\Delta_{2 \nu}\left(a \mid-; q^{2}, t^{2} ; p^{2}\right)} & \text { if } \lambda=2 \nu, \\
0 & \text { otherwise. }\end{cases}
\end{aligned}
$$

Dividing both sides by $\Delta_{\lambda}^{0}\left(a \mid q t / p ; q^{2}, t^{2} ; p^{2}\right)$ and replacing $a \mapsto a t^{2} / q^{2}$ results in (7.8).

The $p \rightarrow 0$ limit of Theorem 7.3 yields a summation generalising Andrews' terminating $q$-analogue of Watson's ${ }_{3} F_{2}$ summation [2, Theorem 1]

$$
{ }_{4} \phi_{3}\left[\begin{array}{cl}
a^{1 / 2},-a^{1 / 2}, b q^{N-1}, q^{-N} \\
a, b^{1 / 2},-b^{1 / 2}
\end{array} ; q, q\right]= \begin{cases}\frac{a^{N / 2}\left(q, b / a ; q^{2}\right)_{N / 2}}{\left(a q, b ; q^{2}\right)_{N / 2}} & \text { if } N \text { is even } \\
0 & \text { otherwise. }\end{cases}
$$


Corollary 7.4. For $\lambda$ a partition,

$$
\begin{aligned}
& \Phi_{\lambda}\left(a^{1 / 2} ;-a^{1 / 2},(a t)^{1 / 2},-(a t)^{1 / 2} ; q, t\right) \\
& \quad= \begin{cases}(a / q)^{|\mu|} t^{-2 n(\mu)} \frac{C_{\mu}^{-}\left(q, q t ; q^{2}, t\right) C_{\mu}^{+}\left(a, a t ; q^{2}, t\right)}{C_{2 \mu^{2}}^{0}\left(a t ; q^{2}, t\right)} & \text { if } \lambda=2 \mu, \\
0 & \text { otherwise. }\end{cases}
\end{aligned}
$$

Andrews' summation is obtained by taking $\lambda=(N)$ and replacing $(a, t) \mapsto(a q, b / a q)$. In Conjecture 9.1 below we give a second multi-sum generalisation of (7.10).

Proof. In the following we consider the right-hand side of $(7.8)$ with $\left(p^{2}, q^{2}, t^{2}\right) \mapsto(p, q, t)$.

By (2.9),

$$
\Delta_{\lambda}(a \mid-; q, t, p)=\left(\frac{t}{a^{3} q^{3}}\right)^{|\lambda|} q^{-4 n\left(\lambda^{\prime}\right)} t^{6 n(\lambda)} \frac{C_{2 \lambda^{2}}^{0}(a q ; q, t ; p)}{C_{\lambda}^{-}(q, t ; q, t ; p) C_{\lambda}^{+}(a, a q / t ; q, t ; p)} .
$$

This implies

$$
\begin{aligned}
\lim _{p \rightarrow 0} & \frac{\Delta_{\mu}\left(a t /\left.q\right|^{-} ; q^{2}, t, p\right)}{\Delta_{2 \mu}\left(a t /\left.q\right|^{-} ; q, t, p\right)} \\
& =\left(a^{3} q t^{2}\right)^{|\mu|} q^{8 n\left(\mu^{\prime}\right)} t^{-6 n(\mu)} \frac{C_{2 \mu^{2}}^{0}\left(a q t ; q^{2}, t\right) C_{2 \mu}^{-}(q, t ; q, t) C_{2 \mu}^{+}(a, a t / q ; q, t)}{C_{4 \mu^{2}}^{0}(a t ; q, t) C_{\mu}^{-}\left(q^{2}, t ; q^{2}, t\right) C_{\mu}^{+}\left(a q, a t / q ; q^{2}, t\right)} \\
& =\left(a^{3} q t^{2}\right)^{|\mu|} q^{8 n\left(\mu^{\prime}\right)} t^{-6 n(\mu)} \frac{C_{\mu}^{-}\left(q, q t ; q^{2}, t\right) C_{\mu}^{+}\left(a, a t ; q^{2}, t\right)}{C_{2 \mu^{2}}^{0}\left(a t ; q^{2}, t\right)}
\end{aligned}
$$

where the last line follows from (2.12a). Also

$$
\frac{1}{\Delta_{2 \mu}^{0}(a t / q \mid b ; q, t ; p)}=(a q t)^{-2|\mu|} q^{-8 n\left(\mu^{\prime}\right)} t^{4 n(\mu)} \frac{1}{\Delta_{2 \mu}^{0}(a t / q \mid b p ; q, t ; p)},
$$

and thus

$$
\lim _{p \rightarrow 0} \frac{1}{\Delta_{2 \mu}^{0}\left(a t / q \mid(q t / p)^{1 / 2} ; q, t ; p\right)}=(a q t)^{-2|\mu|} q^{-8 n\left(\mu^{\prime}\right)} t^{4 n(\mu)} .
$$

The identity (7.11) now follows from Lemma 7.1.

Before stating our second summation formula for $\Phi_{\lambda}$ we remark that if instead of specialising $\boldsymbol{\lambda}=(0, \lambda)$ in $(7.9)$ we take $\boldsymbol{\lambda}=(\lambda, 0)$ and then swap $p$ and $q$, we obtain the following higherdimensional analogue of (2.19a):

$$
\sum_{\mu} \frac{C_{\mu}^{-}\left(p q t ; q^{2}, t^{2} ; p^{2}\right) C_{\mu}^{+}\left(a q^{2} / t^{2} ; q^{2}, t^{2} ; p^{2}\right)}{C_{2 \mu^{2}}^{0}\left(a p^{2} q^{2} ; q^{2}, t^{2} ; p^{4}\right)}\left\langle\begin{array}{l}
\lambda \\
\mu
\end{array}\right\rangle_{[a, p t / q] ; q^{2}, t^{2} ; p^{2}}=\frac{\Delta_{\lambda}\left(a \mid-; q^{2}, t^{2} ; p^{4}\right)}{\Delta_{\lambda}\left(\left.a\right|^{-} ; q^{2}, t^{2} ; p^{2}\right)}
$$

Our second theorem generalises (2.19d), obtained by taking $\lambda=(N)$ and replacing $(a, b) \mapsto$ $\left(-a / b p, b^{2} p q\right)$.

Theorem 7.5. For $\lambda$ a partition,

$$
\Phi_{\lambda}\left(a q ; a,-a q t,-a t ; q^{2}, t^{2} ; p^{2}\right)=\frac{\Delta_{\lambda}(a t / q \mid-; q,-t ; p)}{\Delta_{\lambda}\left(a^{2} t^{2} / q^{2} \mid t / p q ; q^{2}, t^{2} ; p^{2}\right)} .
$$


Proof. The proof is basically the same as that of Theorem 7.3. Inverting (5.18) using (4.42), we have

$$
\sum_{\boldsymbol{\mu}} \frac{C_{\boldsymbol{\mu}}^{-}\left(p q t ; t^{2} ; p^{2}, q^{2}\right) C_{\boldsymbol{\mu}}^{+}\left(a^{2} p^{2} q^{2} / t^{2} ; t^{2} ; p^{2}, q^{2}\right)}{C_{2 \boldsymbol{\mu}^{2}}^{0}(-a p q ;-t ; p, q)}\left\langle\begin{array}{l}
\boldsymbol{\lambda} \\
\boldsymbol{\mu}
\end{array}\right\rangle_{\left[a^{2}, t / p q\right] ; t^{2} ; p^{2}, q^{2}}=\frac{\Delta_{\boldsymbol{\lambda}}(a \mid-;-t ; p, q)}{\Delta_{\boldsymbol{\lambda}}\left(a^{2} \mid-; t^{2} ; p^{2}, q^{2}\right)} .
$$

Taking $\boldsymbol{\lambda}=(0, \lambda)$, noting that by $(2.12)$

$$
C_{2 \mu^{2}}^{0}(-a p q ; q,-t ; p)=C_{\mu}^{0}\left(-a p q,-a p q^{2}, a p q / t, a p q^{2} / t ; q^{2}, t^{2} ; p\right),
$$

and finally dividing both sides by $\Delta_{\lambda}^{0}\left(a^{2} \mid t / p q ; q^{2}, t^{2} ; p^{2}\right)$, we obtain (7.12) with $a \mapsto a q / t$.

The $p \rightarrow 0$ limit of (7.12) yields a generalisation of the following quadratic summation due to Bressoud, Ismail and Stanton [4, equation (2.1)]:

$$
{ }_{4} \phi_{3}\left[\begin{array}{c}
a, a q, b^{2} q^{2 N-2}, q^{-2 N} \\
a^{2}, b, b q
\end{array} q^{2}, q^{2}\right]=\frac{a^{N}(1-b / q)(-q, b / a ; q)_{N}}{\left(1-b q^{2 N-1}\right)(-a, b / q ; q)_{N}} .
$$

Corollary 7.6. For $\lambda$ a partition,

$$
\Phi_{\lambda}\left(a q ; a, a q t, a t ; q^{2}, t^{2}\right)=(-a)^{|\lambda|} t^{-2 n(\lambda)} \frac{C_{\lambda}^{-}(-q,-t ; q, t) C_{\lambda}^{+}(a, a t / q ; q, t)}{C_{2 \lambda^{2}}^{0}(a t ; q, t)} .
$$

We note that for $\lambda=(N)$ and $(a, t) \mapsto(-a, b / a)$ this is (7.13) and that in going from (7.12) to (7.14) the parameter $t$ has been replaced by $-t$.

The proof of Corollary 7.6 proceeds along the same lines as the proof of Corollary 7.4, and we omit the details.

\section{Proof of Theorem 1.2}

In Section 1 we stated (1.12) as a corollary of (1.8), but in fact both results are equivalent, and proving (1.12) for fixed $m, r$ and all $n \geqslant r$ is the same as proving (1.8) for fixed $m, r$. To avoid the use of virtual Koornwinder polynomials in our proof, we will in the following establish Corollary 1.3 instead of Theorem 1.2.

The first step in our proof is to dualise the three claims of Corollary 1.3, an approach that was also utilised in [42] to prove bounded Littlewood identities for Macdonald polynomials.

Let $x:=\left(x_{1}, \ldots, x_{n}\right)$ and $y:=\left(y_{1}, \ldots, y_{m}\right)$. By the complementation symmetry (4.8) and homogeneity of the Macdonald polynomials, the (dual) Cauchy identity (4.6) can be written in the form (see also [32])

$$
\sum_{\mu \subset\left(m^{n}\right)}(-1)^{|\mu|} P_{\left(m^{n}\right)-\mu}(x ; q, t) P_{\mu^{\prime}}(y ; t, q)=\prod_{i=1}^{n} \prod_{j=1}^{m}\left(x_{i}-y_{j}\right) \text {. }
$$

Replacing $n \mapsto 2 n$ and then specialising $x_{i+n}=x_{i}^{-1}$ for all $1 \leqslant i \leqslant n$ yields

$$
\sum_{\mu \subset\left(m^{2 n}\right)}(-1)^{|\mu|} P_{\left(m^{2 n}\right)-\mu}\left(x^{ \pm} ; q, t\right) P_{\mu^{\prime}}(y ; t, q)=\prod_{i=1}^{n} \prod_{j=1}^{m} x_{i}^{-1}\left(x_{i}-y_{j}\right)\left(1-x_{i} y_{j}\right) .
$$

Up to the simple factor $(-1)^{m n}\left(y_{1} \cdots y_{m}\right)^{n}$ the right-hand side coincides with the right-hand side of the Cauchy identity (4.26) for Koornwinder polynomials. Correcting for this factor, we 
can thus equate the respective left-hand sides, resulting in

$$
\begin{aligned}
& \sum_{\mu \subset\left(m^{2 n}\right)}(-1)^{|\mu|} P_{\left(m^{2 n}\right)-\mu}\left(x^{ \pm} ; q, t\right) P_{\mu^{\prime}}(y ; t, q) \\
& \quad=\sum_{\nu \subset\left(m^{n}\right)}(-1)^{m n+|\nu|} K_{\left(m^{n}\right)-\nu}(x ; q, t ; \underline{t})\left(y_{1} \cdots y_{m}\right)^{n} K_{\nu^{\prime}}(y ; t, q ; \underline{t}) .
\end{aligned}
$$

Let $r$ be an integer such that $0 \leqslant r \leqslant n, \lambda$ a partition contained in $\left(m^{r}\right)$, and $s:=2 n-r \geqslant n$. Extracting the coefficient of

$$
K_{\left(m^{r}\right)-\lambda}(x ; q, t ; \underline{t}) P_{\left(s^{m}\right)}(y ; t, q)
$$

in (8.1) picks out the term $\mu=\left(m^{s}\right)$ in the sum on the left and

$$
\nu=(\underbrace{m, \ldots, m}_{n-r \text { times }}, \lambda_{1}, \ldots, \lambda_{r})
$$

in the sum on the right. For such $\mu$ and $\nu$,

$$
\left(m^{2 n}\right)-\mu=\left(m^{r}\right) \quad \text { and } \quad \nu^{\prime}=\lambda^{\prime}+\left(N^{m}\right),
$$

where $N:=n-r$. Hence

$$
\begin{aligned}
& {\left[K_{\left(m^{r}\right)-\lambda}(x ; q, t ; \underline{t})\right] P_{\left(m^{r}\right)}\left(x^{ \pm} ; q, t\right)} \\
& \quad=(-1)^{|\lambda|}\left[P_{\left(s^{m}\right)}(y ; t, q)\right]\left(y_{1} \cdots y_{m}\right)^{n} K_{\lambda^{\prime}+\left(N^{m}\right)}(y ; t, q ; \underline{t}),
\end{aligned}
$$

where the reader is warned that in the above right-hand side we have not followed our earlier convention, and $\left[P_{\lambda}(y ; t, q)\right] f(y)$ denotes the coefficient of $P_{\lambda}(t, q)$ of $f \in \Lambda_{\mathrm{GL}(m)}$.

Recall that in (4.7) we extended the Macdonald polynomials to arbitrary weights. Accordingly, for $k \in \mathbb{Z}$ and $\mu \in P(m)$,

$$
P_{\mu}(y ; q, t)=\left(y_{1} \cdots y_{m}\right)^{k} P_{\left(\mu_{1}-k, \ldots, \mu_{m}-k\right)}(y ; q, t) .
$$

This implies that if $f$ is an $S_{m}$-symmetric Laurent polynomial in $y$, then

$$
\left[P_{\mu}(y ; t, q)\right]\left(y_{1} \cdots y_{m}\right)^{k} f(y)=\left[P_{\left(\mu_{1}-k, \ldots, \mu_{m}-k\right)}(y ; t, q)\right] f(y) .
$$

Equation (8.2) therefore simplifies to

$$
\left[K_{\left(m^{r}\right)-\lambda}(x ; q, t ; \underline{t})\right] P_{\left(m^{r}\right)}\left(x^{ \pm} ; q, t\right)=(-1)^{|\lambda|}\left[P_{\left(N^{m}\right)}(y ; t, q)\right] K_{\lambda^{\prime}+\left(N^{m}\right)}(y ; t, q ; \underline{t}),
$$

where we recall that $N:=n-r \geqslant 0$. Comparing this with (1.12) (where $P^{\left(\mathrm{B}_{n}, \mathrm{C}_{n}\right)}(q, t, s)$ and $P^{\left(\mathrm{C}_{n}, \mathrm{C}_{n}\right)}(q, t, s)$ are given by $\left.(1.5)\right)$ it follows that we must prove three identities for

$$
\left[P_{\left(N^{m}\right)}(y ; t, q)\right] K_{\lambda^{\prime}+\left(N^{m}\right)}(y ; t, q ; \underline{t}) .
$$

(Of course, by duality we only need to actually prove two identities.) To state these in the simplest possible form we first define, in analogy with (6.2),

$$
d_{\lambda \mu}^{(n)}(q, t ; \underline{t}):=\left[P_{\lambda}(x ; q, t)\right] K_{\mu}(x ; q, t ; \underline{t}) \in \mathbb{Q}\left(q, t, t_{0}, t_{1}, t_{2}, t_{3}\right)
$$

for $\lambda \in P(n), \mu \in P_{+}(n)$. By (4.25), (6.1) and the homogeneity of the Macdonald polynomials, these coefficients satisfy the symmetries

$$
d_{\lambda \mu}^{(n)}(q, t ; \underline{t})=d_{\lambda \mu}^{(n)}(1 / q, 1 / t ; 1 / \underline{t})=(-1)^{|\lambda|-|\mu|} d_{\lambda \mu}^{(n)}(q, t ;-\underline{t})=d_{\left(-\lambda_{n}, \ldots,-\lambda_{1}\right), \mu}^{(n)}(q, t ; \underline{t}),
$$


where $\lambda=\left(\lambda_{1}, \ldots, \lambda_{n}\right)$.

In the case of $(1.12 \mathrm{a})$ we need to show that

$$
d_{\left(N^{m}\right), \lambda^{\prime}+\left(N^{m}\right)}^{(m)}\left(t, q ; t^{1 / 2},-t^{1 / 2},(q t)^{1 / 2},-(q t)^{1 / 2}\right)= \begin{cases}c_{\lambda}\left(q^{-m}, t^{-N} ; q, t\right) & \text { if } \lambda^{\prime} \text { even } \\ 0 & \text { otherwise }\end{cases}
$$

where $N$ is an arbitrary nonnegative integer and $\lambda \in P_{+}(m)$. Replacing

$$
\left(m, \lambda, y_{1}, \ldots, y_{m}, q, t\right) \mapsto\left(n, \lambda^{\prime}, x_{1}, \ldots, x_{n}, t, q\right)
$$

and defining

$$
\hat{c}_{2 \lambda}(w, z ; q, t):=c_{\left(\lambda^{\prime}\right)^{2}}\left(z^{-1}, w^{-1} ; t, q\right),
$$

this can be rewritten as

$$
d_{\left(N^{n}\right), \lambda+\left(N^{n}\right)}^{(n)}\left(q, t ; q^{1 / 2},-q^{1 / 2},(q t)^{1 / 2},-(q t)^{1 / 2}\right)= \begin{cases}\hat{c}_{\lambda}\left(q^{N}, t^{n} ; q, t\right) & \text { if } \lambda \text { even, } \\ 0 & \text { otherwise }\end{cases}
$$

where now $\lambda \in P_{+}(n)$. By (1.9a) and (2.10),

$$
\hat{c}_{2 \lambda}(w, z ; q, t)=\frac{C_{2 \lambda}^{0}(z ; q, t)}{C_{2 \lambda}^{0}(q z / t ; q, t)} \frac{C_{\lambda}^{-}\left(q ; q^{2}, t\right)}{C_{\lambda}^{-}\left(t ; q^{2}, t\right)} \frac{C_{\lambda}^{+}\left(q^{2} w^{2} z^{2} / t^{2} ; q^{2}, t\right)}{C_{\lambda}^{+}\left(q w^{2} z^{2} / t ; q^{2}, t\right)} .
$$

Similarly, the dual case of $(1.12 \mathrm{~b})$ translates to

$$
d_{\left(N^{n}\right), \lambda+\left(N^{n}\right)}^{(n)}\left(q, t ; 1,-1, q^{1 / 2},-q^{1 / 2}\right)= \begin{cases}\hat{d}_{\lambda}\left(q^{N}, t^{n} ; q, t\right) & \text { if } \lambda^{\prime} \text { even } \\ 0 & \text { otherwise }\end{cases}
$$

with $N$ and $\lambda$ as above, and

$$
\hat{d}_{\lambda^{2}}(w, z ; q, t)=d_{2 \lambda^{\prime}}\left(z^{-1}, w^{-1} ; t, q\right)=\frac{C_{\lambda^{2}}^{0}(z ; q, t)}{C_{\lambda^{2}}^{0}(q z / t ; q, t)} \frac{C_{\lambda}^{-}\left(q t ; q, t^{2}\right)}{C_{\lambda}^{-}\left(t^{2} ; q, t^{2}\right)} \frac{C_{\lambda}^{+}\left(q w^{2} z^{2} / t^{4} ; q, t^{2}\right)}{C_{\lambda}^{+}\left(w^{2} z^{2} / t^{3} ; q, t^{2}\right)}
$$

Finally, in the case of $(1.12 \mathrm{c})$ we get

$$
d_{\left(N^{n}\right), \lambda+\left(N^{n}\right)}^{(n)}\left(q^{2}, t^{2} ;-1,-q,-t,-q t\right)=\hat{e}_{\lambda}\left(q^{N}, t^{n} ; q, t\right),
$$

where

$$
\begin{aligned}
\hat{e}_{\lambda}(w, z ; q, t) & =(-1)^{|\lambda|} e_{\lambda^{\prime}}\left(z^{-1}, w^{-1} ; t, q\right) \\
& =\frac{C_{\lambda}^{0}\left(z^{2} ; q^{2}, t^{2}\right)}{C_{\lambda}^{0}\left(q^{2} z^{2} / t^{2} ; q^{2}, t^{2}\right)} \frac{C_{\lambda}^{-}(-q ; q, t)}{C_{\lambda}^{-}(t ; q, t)} \frac{C_{\lambda}^{+}\left(q w^{2} z^{2} / t^{2} ; q, t\right)}{C_{\lambda}^{+}\left(-w^{2} z^{2} / t ; q, t\right)} .
\end{aligned}
$$

By (8.4) we also have the companion identity

$$
d_{\left(N^{n}\right), \lambda+\left(N^{n}\right)}^{(n)}\left(q^{2}, t^{2} ; 1, q, t, q t\right)=(-1)^{|\lambda|} \hat{e}_{\lambda}\left(q^{N}, t^{n} ; q, t\right) .
$$

In the following it will be convenient to define

$$
\begin{aligned}
\mathcal{D}_{\lambda}^{(N, n)}\left(q, t ; t_{0}:\right. & \left.t_{1}, t_{2}, t_{3}\right):=\left(t_{0} q^{N} t^{n-1}\right)^{|\lambda|} t^{-n(\lambda)} d_{\left(N^{n}\right), \lambda+\left(N^{n}\right)}^{(n)}(q, t ; \underline{t}) \\
& \times \frac{C_{\lambda}^{0}\left(q t^{n-1} ; q, t\right) C_{\lambda}^{-}(t ; q, t) C_{\lambda}^{+}\left(t_{0} t_{1} t_{2} t_{3} q^{2 N-1} t^{2 n-2} ; q, t\right)}{C_{\lambda}^{0}\left(t^{n}, q^{N+1} t^{n-1}, t_{0} t_{1} q^{N} t^{n-1}, t_{0} t_{2} q^{N} t^{n-1}, t_{0} t_{3} q^{N} t^{n-1} ; q, t\right)},
\end{aligned}
$$


for $\lambda \in P_{+}(n)$. The colon between $t_{0}$ and $t_{1}, t_{2}, t_{3}$ indicates the absence of full $S_{4}$-symmetry. Combining (4.24) with Corollary 6.2 then gives

$$
\begin{aligned}
\mathcal{D}_{\lambda}^{(N, n)}\left(q, t ; t_{0}: t_{1}, t_{2}, t_{3}\right) & \\
=\sum_{\mu \subset \lambda} & \left((-1)^{|\mu|} q^{-n\left(\mu^{\prime}\right)} t^{n(\mu)} \frac{C_{\mu}^{0}\left(q t^{n-1} ; q, t\right) C_{\mu}^{-}(t ; q, t) C_{\mu}^{+}\left(t_{0} t_{1} t_{2} t_{3} q^{2 N-1} t^{2 n-2} ; q, t\right)}{C_{\mu}^{0}\left(t^{n}, q^{N+1} t^{n-1}, t_{0} t_{1} q^{N} t^{n-1}, t_{0} t_{2} q^{N} t^{n-1}, t_{0} t_{3} q^{N} t^{n-1} ; q, t\right)}\right. \\
& \left.\times\left[\begin{array}{l}
\lambda \\
\mu
\end{array}\right]_{q, t,\left(t_{0} t_{1} t_{2} t_{3} q^{2 N-1} t^{2 n-2}\right)^{1 / 2}} C_{\left(N^{n}\right), \mu+\left(N^{n}\right)}^{(n)}\left(q, t, t_{0}\right)\right),
\end{aligned}
$$

where we have also used (2.13) (with $p=0$ ) and (4.22), as well as the definitions (6.2) and (6.3). Recalling that $c_{\lambda, \mu}^{(n)}\left(q, t, t_{0}\right)$ is a function of $t_{0}^{2}$, it follows from (6.7) that

$$
\begin{aligned}
\mathcal{D}_{\lambda}^{(N, n)}\left(q, t ;-q^{1 / 2}:-q^{1 / 2} t_{1},-q^{1 / 2} t_{2},-q^{1 / 2} t_{3}\right) & \\
= & \sum_{\mu \subset \lambda}\left((-1)^{|\mu|} q^{-n\left(\mu^{\prime}\right)} t^{n(\mu)}\left[\begin{array}{l}
\lambda \\
\mu
\end{array}\right]_{q, t,\left(t_{1} t_{2} t_{3} q^{2 N+1} t^{2 n-2}\right)^{1 / 2}}\right. \\
& \left.\times \frac{C_{\mu}^{+}\left(q^{2 N+1} t^{2 n-2}, t_{1} t_{2} t_{3} q^{2 N-1} t^{2 n-2} ; q, t\right)}{C_{\mu}^{0}\left(q^{N+1} t^{n-1}, t_{1} q^{N+1} t^{n-1}, t_{2} q^{N+1} t^{n-1}, t_{3} q^{N+1} t^{n-1} ; q, t\right)}\right) \\
= & \Phi_{\lambda}\left(q^{N+1} t^{n-1} ; t_{1} q^{N+1} t^{n-1}, t_{2} q^{N+1} t^{n-1}, t_{3} q^{N+1} t^{n-1} ; q, t\right),
\end{aligned}
$$

with $\Phi_{\lambda}$ defined in (7.2). If we specialise $\left\{t_{1}, t_{2}, t_{3}\right\}=\left\{-1, t^{1 / 2},-t^{1 / 2}\right\}$ then, by Corollary 7.4,

$$
\begin{aligned}
& \mathcal{D}_{\lambda}^{(N, n)}\left(q, t ;-q^{1 / 2}: q^{1 / 2},-(q t)^{1 / 2},(q t)^{1 / 2}\right) \\
& \quad=\left(q^{2 N+1} t^{2 n-2}\right)^{|\mu|} t^{-2 n(\mu)} \frac{C_{\mu}^{-}\left(q, q t ; q^{2}, t\right) C_{\mu}^{+}\left(q^{2 N+2} t^{2 n-2}, q^{2 N+2} t^{2 n-1} ; q^{2}, t\right)}{C_{2 \mu^{2}}^{0}\left(q^{2 N+2} t^{2 n-1} ; q^{2}, t\right)}
\end{aligned}
$$

if $\lambda=2 \mu$ and 0 otherwise. By (8.8) the non-vanishing case finally gives

$$
\begin{aligned}
& d_{\left(N^{n}\right), \lambda+\left(N^{n}\right)}^{(n)}\left(q, t ; q^{1 / 2},-q^{1 / 2},(q t)^{1 / 2},-(q t)^{1 / 2}\right) \\
& \quad=\frac{C_{2 \mu}^{0}\left(t^{n} ; q, t\right) C_{\mu}^{-}\left(q ; q^{2}, t\right) C_{\mu}^{+}\left(q^{2 N+2} t^{2 n-2} ; q^{2}, t\right)}{C_{2 \mu}^{0}\left(q t^{n-1} ; q, t\right) C_{\mu}^{-}\left(t ; q^{2}, t\right) C_{\mu}^{+}\left(q^{2 N+1} t^{2 n-1} ; q^{2}, t\right)} \\
& \quad=\hat{c}_{\lambda}\left(q^{N}, t^{n} ; q, t\right),
\end{aligned}
$$

where we have also used (2.12) to simplify the $C$-symbols in the second line. This completes the proof of (8.5) and, by duality, that of (8.6).

To prove the identity (8.7), we replace $(q, t) \mapsto\left(q^{2}, t^{2}\right)$ in (8.9) and then make the specialisation $\left\{t_{1}, t_{2}, t_{3}\right\}=\{1 / q, t, t / q\}$. This gives

$$
\begin{aligned}
\mathcal{D}_{\lambda}^{(N, n)} & \left(q^{2}, t^{2} ;-q:-1,-t,-q t\right) \\
& =\Phi_{\lambda}\left(q^{2 N+2} t^{2 n-2} ; q^{2 N+1} t^{2 n-2}, q^{2 N+2} t^{2 n-1}, q^{2 N+1} t^{2 n-1} ; q^{2}, t^{2}\right) .
\end{aligned}
$$

By Corollary 7.6 this series on the right once again can be summed to yield

$$
\begin{aligned}
\mathcal{D}_{\lambda}^{(N, n)} & \left(q^{2}, t^{2} ;-q:-1,-q t,-t\right) \\
& =\left(-q^{2 N+1} t^{2 n-2}\right)^{|\lambda|} t^{-2 n(\lambda)} \frac{C_{\lambda}^{-}(-q,-t ; q, t) C_{\lambda}^{+}\left(q^{2 N+1} t^{2 n-2}, q^{2 N} t^{2 n-1} ; q, t\right)}{C_{2 \lambda^{2}}^{0}\left(q^{2 N+1} t^{2 n-1} ; q, t\right)},
\end{aligned}
$$

and hence by (8.8) and (2.12),

$$
\begin{aligned}
d_{\left(N^{n}\right), \lambda+\left(N^{n}\right)}^{(n)}\left(q^{2}, t^{2} ;-1,-q,-t,-q t\right) & =\frac{C_{\lambda}^{0}\left(t^{2 n} ; q^{2}, t^{2}\right) C_{\lambda}^{-}(-q ; q, t) C_{\lambda}^{+}(a / q ; q, t)}{C_{\lambda}^{0}\left(q^{2} t^{2 n-2} ; q^{2}, t^{2}\right) C_{\lambda}^{-}(t ; q, t) C_{\lambda}^{+}\left(-a t / q^{2} ; q, t\right)} \\
& =\hat{e}_{\lambda}\left(q^{N}, t^{n} ; q, t\right) .
\end{aligned}
$$




\section{Open problems}

The problem with proving the branching rule of Conjecture 1.4 is that it requires the following curious identity for the basic hypergeometric function

$$
\Phi_{\lambda}\left((a q)^{1 / 2} ;-(a q)^{1 / 2},(a t)^{1 / 2},-(a t)^{1 / 2} ; q, t\right) .
$$

Conjecture 9.1. For $\lambda$ a partition

$$
\begin{aligned}
& \sum_{\mu \subset \lambda}(-1)^{|\mu|} q^{-n\left(\mu^{\prime}\right)} t^{n(\mu)} \frac{C_{\mu}^{+}(a, a t / q ; q, t)}{C_{\mu}^{0}\left(a q, a t ; q^{2}, t^{2}\right)}\left[\begin{array}{l}
\lambda \\
\mu
\end{array}\right]_{q, t,(a t / q)^{1 / 2}} \\
& = \begin{cases}a^{|\lambda| / 2} q^{2 \hat{n}^{\circ}\left(\lambda^{\prime}\right)-2 \hat{n}^{\mathrm{e}}\left(\lambda^{\prime}\right)} t^{-2 n^{\circ}(\lambda)} \frac{C_{\lambda}^{-, \mathrm{e}}(q, t ; q, t) C_{\lambda}^{+, \mathrm{e}}(a, a t / q ; q, t)}{C_{\lambda}^{0}\left(a q, a t ; q^{2}, t^{2}\right)} & \text { if } 2 \text {-core }(\lambda)=0, \\
0 & \text { otherwise. }\end{cases}
\end{aligned}
$$

For $\lambda=(N)$ this is (7.10) with $b$ replaced by at. By the $p=0$ case of (7.5) it follows that, up to a rescaling of $a,(9.1)$ is invariant under conjugation of $\lambda^{\prime}$. Hence it also holds for $\lambda=\left(1^{n}\right)$. Since the elliptic hypergeometric series

$$
\Phi_{\lambda}\left((a q)^{1 / 2} ;-(a q)^{1 / 2},(a t)^{1 / 2},-(a t)^{1 / 2} ; q, t ; p\right)
$$

is not very-well poised, it remains unclear what the elliptic analogue Conjecture 9.1 should be. Another obvious special case arises when $t=q$, in which case we can use the determinantal expression (4.23) for the generalised binomial coefficients. Up to an overall factor, the left-hand side of (9.1) for $a \mapsto a t^{2 n-2}$ may then be written as

$$
\sum_{\nu_{1}, \ldots, \nu_{n}=0}^{\kappa_{1}} \prod_{1 \leqslant i<j \leqslant n} \frac{\left(q^{\nu_{i}}-q^{\nu_{j}+1}\right)}{\left(1-a q^{\nu_{i}+\nu_{j}}\right)} \operatorname{det}_{1 \leqslant i, j \leqslant n}\left(\frac{\left(a q^{\kappa_{i}}, q^{-\kappa_{i}} ; q\right)_{\nu_{j}}\left(a ; q^{2}\right)_{\nu_{j}}}{(q, a ; q)_{\nu_{j}}\left(a q ; q^{2}\right)_{\nu_{j}}} q^{\nu_{j}}\right),
$$

where $\kappa_{i}:=\lambda_{i}+n-i$. It is again unclear why this vanishes unless $\lambda \in P_{+}(n)$ has empty 2-core.

We conclude with several conjectures closely related to Conjecture 9.1, such as new vanishing integrals in the sense of $[35,41]$ and a number of new Littlewood-type identities.

Let

$$
\mathrm{d} T(x):=\frac{1}{2^{n} n !(2 \pi \mathrm{i})^{n}} \frac{\mathrm{d} x_{1}}{x_{1}} \cdots \frac{\mathrm{d} x_{n}}{x_{n}}
$$

and, for $|a|,|b|,|q|,|t|<1$,

$$
\begin{aligned}
Z_{n}(a, b ; q, t) & :=\int_{\mathbb{T}^{n}} \prod_{i=1}^{n} \frac{\left(x_{i}^{ \pm 2} ; q\right)_{\infty}}{\left(a x_{i}^{ \pm 2}, b x_{i}^{ \pm 2} ; q^{2}\right)_{\infty}} \prod_{1 \leqslant i<j \leqslant n} \frac{\left(x_{i}^{ \pm} x_{j}^{ \pm} ; q\right)_{\infty}}{\left(t x_{i}^{ \pm} x_{j}^{ \pm} ; q\right)_{\infty}} \mathrm{d} T(x) \\
& =\prod_{i=1}^{n} \frac{\left(t, a b t^{n+i-2} ; q\right)_{\infty}}{\left(q, t^{i},-a t^{i-1},-b t^{i-1} ; q\right)_{\infty}\left(a b t^{2 i-2} ; q^{2}\right)_{\infty}^{2}}
\end{aligned}
$$

where the explicit evaluation is a special case of Gustafson's $\mathrm{BC}_{n}$ analogue of the Askey-Wilson integral [14].

Conjecture 9.2 (vanishing integral). Let $\lambda \in P_{+}(2 n)$ and $a, b, q, t \in \mathbb{C}$ such that $|a|,|b|,|q|$, $|t|<1$. Then

$$
\begin{aligned}
& I_{\lambda}\left(a, b ; q, t, t^{n}\right):=\frac{1}{Z_{n}(a, b ; q, t)} \\
& \quad \times \int_{\mathbb{T}^{n}} P_{\lambda}\left(x_{1}^{ \pm}, \ldots, x_{n}^{ \pm} ; q, t\right) \prod_{i=1}^{n} \frac{\left(x_{i}^{ \pm 2} ; q\right)_{\infty}}{\left(a x_{i}^{ \pm 2}, b x_{i}^{ \pm 2} ; q^{2}\right)_{\infty}} \prod_{1 \leqslant i<j \leqslant n} \frac{\left(x_{i}^{ \pm} x_{j}^{ \pm} ; q\right)_{\infty}}{\left(t x_{i}^{ \pm} x_{j}^{ \pm} ; q\right)_{\infty}} \mathrm{d} T(x)
\end{aligned}
$$


vanishes unless 2 -core $(\lambda)=0$. Moreover

$$
I_{\lambda}(q, t ; q, t, T)=q^{n^{\mathrm{e}}\left(\lambda^{\prime}\right)-n^{\mathrm{o}}\left(\lambda^{\prime}\right)} t^{2 \hat{n}^{\mathrm{o}}(\lambda)-2 \hat{n}^{\mathrm{e}}(\lambda)} \frac{C_{\lambda}^{0, \mathrm{e}}\left(T^{2} ; q, t\right)}{C_{\lambda}^{0, \mathrm{o}}\left(q T^{2} / t ; q, t\right)} \frac{C_{\lambda}^{-, \mathrm{e}}(q ; q, t)}{C_{\lambda}^{-, \mathrm{o}}(t ; q, t)}
$$

and

$$
I_{\lambda}(1, q t ; q, t, T)=\frac{u_{\lambda}(q, t)+v_{\lambda}(q, t) T}{1+T} \frac{C_{\lambda}^{0, \mathrm{e}}\left(T^{2} ; q, t\right)}{C_{\lambda}^{0, \mathrm{o}}\left(q T^{2} / t ; q, t\right)} \frac{C_{\lambda}^{-, \mathrm{e}}(q ; q, t)}{C_{\lambda}^{-, \mathrm{o}}(t ; q, t)},
$$

where

$$
\begin{aligned}
& u_{\lambda}(q, t):=q^{2 \hat{n}^{\circ}\left(\lambda^{\prime}\right)-2 \hat{n}^{\mathrm{e}}\left(\lambda^{\prime}\right)} t^{n^{\mathrm{e}}(\lambda)-n^{\circ}(\lambda)},
\end{aligned}
$$

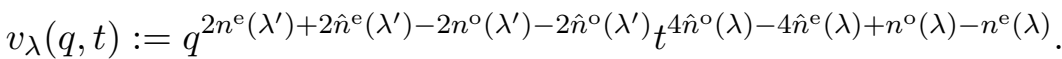

That the integral on the right of (9.2) depends on $n$ only through $t^{n}$ follows from the fact that this integral is equal to the $T=t^{n}$ instance of

$$
\left[\tilde{K}_{0}\left(x_{1}, \ldots, x_{n} ; q, t, T ; a^{1 / 2},-a^{1 / 2}, b^{1 / 2},-b^{1 / 2}\right)\right] P_{\lambda}\left(x_{1}^{ \pm}, \ldots, x_{n}^{ \pm} ; q, t\right)
$$

with $\tilde{K}_{\lambda}$ the lifted Koornwinder polynomial (4.27), see [35].

When $t=q$ we have a proof of the vanishing part of the conjecture and alternative expressions for the right-hand sides of $(9.3 \mathrm{a})$ and $(9.3 \mathrm{~b})$ in terms of pfaffians. Define $I_{\lambda}(a, b ; q, T):=$ $I_{\lambda}(a, b ; q, q, T)$ and $Z_{n}(a, b ; q):=Z_{n}(a, b ; q, q)$.

Proposition 9.3. Let $\lambda \in P_{+}(2 n)$ and $a, b, q \in \mathbb{C}$ such that $|a|,|b|,|q|<1$. Then

$$
\int_{\mathbb{T}^{n}} s_{\lambda}\left(x_{1}^{ \pm}, \ldots, x_{n}^{ \pm}\right) \prod_{i=1}^{n} \frac{\left(x_{i}^{ \pm 2} ; q\right)_{\infty}}{\left(a x_{i}^{ \pm 2}, b x_{i}^{ \pm 2} ; q^{2}\right)_{\infty}} \prod_{1 \leqslant i<j \leqslant n}\left(1-x_{i}^{ \pm} x_{j}^{ \pm}\right) \mathrm{d} T(x)
$$

vanishes unless $\lambda$ has empty 2-core. Moreover,

$$
\begin{aligned}
I_{\lambda}\left(q, q ; q, q^{n}\right)= & \prod_{i=1}^{n} \frac{\left(1-q^{2 i-1}\right)^{2 n-2 i+1}}{\left(1-q^{2 i}\right)^{2 n-2 i}} \\
& \times \operatorname{pf}_{1 \leqslant i, j \leqslant 2 n}\left(\frac{q^{\left(\lambda_{i}-\lambda_{j}-i+j-1\right) / 2}}{1-q^{\lambda_{i}-\lambda_{j}-i+j}} \chi\left(\lambda_{i}-\lambda_{j}-i+j \text { is odd }\right)\right)
\end{aligned}
$$

and

$$
\begin{aligned}
I_{\lambda}\left(1, q^{2} ; q, q^{n}\right)= & \frac{1}{2^{n}} \frac{2}{1+q^{n}} \prod_{i=1}^{n} \frac{\left(1-q^{2 i-1}\right)^{2 n-2 i+1}}{\left(1-q^{2 i}\right)^{2 n-2 i}} \\
& \times \operatorname{pf}_{1 \leqslant i, j \leqslant 2 n}\left(\frac{1+q^{\lambda_{i}-\lambda_{j}-i+j}}{1-q^{\lambda_{i}-\lambda_{j}-i+j}} \chi\left(\lambda_{i}-\lambda_{j}-i+j \text { is odd }\right)\right) .
\end{aligned}
$$

Before we prove this, we remark that for any fixed choice of partition $\lambda$ with empty 2-core each of the above two pfaffians can be written as an $n \times n$ determinant containing the non-vanishing entries, up to sign. For example,

$$
\begin{aligned}
I_{\left(r^{2 n}\right)}\left(q, q ; q, q^{n}\right) & =\prod_{i=1}^{n} \frac{\left(1-q^{2 i-1}\right)^{2 n-2 i+1}}{\left(1-q^{2 i}\right)^{2 n-2 i}} \operatorname{pf}_{1 \leqslant i, j \leqslant 2 n}\left(\frac{q^{(j-i-1) / 2}}{1-q^{j-i}} \chi(j-i \text { is odd })\right) \\
& =\prod_{i=1}^{n} \frac{\left(1-q^{2 i-1}\right)^{2 n-2 i+1}}{\left(1-q^{2 i}\right)^{2 n-2 i}} \operatorname{det}_{1 \leqslant i, j \leqslant n}\left(\frac{(-1)^{j-i} q^{j-i}}{1-q^{2 j-2 i+1}}\right) \\
& =1 .
\end{aligned}
$$


We do not know, however, how to compute the pfaffian for arbitrary $\lambda$. Of course, from (9.3a) it follows that we must have

$$
\begin{aligned}
& \prod_{i=1}^{n} \frac{\left(1-q^{2 i-1}\right)^{2 n-2 i+1}}{\left(1-q^{2 i}\right)^{2 n-2 i}} \operatorname{pf}_{1 \leqslant i, j \leqslant 2 n}\left(\frac{q^{\left(\lambda_{i}-\lambda_{j}-i+j-1\right) / 2}}{1-q^{\lambda_{i}-\lambda_{j}-i+j}} \chi\left(\lambda_{i}-\lambda_{j}-i+j \text { is odd }\right)\right) \\
& =q^{n^{\mathrm{e}}\left(\lambda^{\prime}\right)-n^{\mathrm{o}}\left(\lambda^{\prime}\right)+2 \hat{n}^{\mathrm{o}}(\lambda)-2 \hat{n}^{\mathrm{e}}(\lambda)} \frac{C_{\lambda}^{0, \mathrm{e}}\left(q^{2 n} ; q, q\right)}{C_{\lambda}^{0, \mathrm{o}}\left(q^{2 n} ; q, q\right)} \frac{C_{\lambda}^{-, \mathrm{e}}(q ; q, q)}{C_{\lambda}^{-, \mathrm{o}}(q ; q, q)},
\end{aligned}
$$

with a similar result for the second pfaffian.

Proof of Proposition 9.3. Using (3.1) to express the Schur function $s_{\lambda}$ as a determinant, we have

$$
I_{\lambda}\left(a, b ; q, q^{n}\right)=\frac{1}{Z_{n}(a, b ; q)} \int_{\mathbb{T}^{n}} \operatorname{det}_{1 \leqslant i, j \leqslant 2 n}\left(y_{i}^{\lambda_{j}+2 n-j}\right) \prod_{i=1}^{n} \frac{x_{i}^{-1} \theta\left(x_{i}^{2} ; q\right)}{\left(a x_{i}^{ \pm 2}, b x_{i}^{ \pm 2} ; q^{2}\right)_{\infty}} \mathrm{d} T(x),
$$

where $y=\left(x_{1}, x_{1}^{-1}, \ldots, x_{n}, x_{n}^{-1}\right)$. Let $\left(a_{i j}(x)\right)$ be a $2 n \times 2 n$ matrix such that $a_{2 i-1, j}(x)=\phi_{j}\left(x_{i}\right)$ and $a_{2 i, j}(x)=\psi_{j}\left(x_{i}\right)$. Then [6, equation (7.3)]

$$
\frac{1}{n !} \int \operatorname{det}_{1 \leqslant i, j \leqslant 2 n}\left(a_{i j}(x)\right) \mathrm{d} \mu\left(x_{1}\right) \cdots \mathrm{d} \mu\left(x_{n}\right)=\operatorname{pf}_{1 \leqslant i, j \leqslant 2 n}\left(\int\left(\phi_{i}(x) \psi_{j}(x)-\phi_{j}(x) \psi_{i}(x)\right) \mathrm{d} \mu(x)\right) .
$$

Applying this to (9.6) yields

$$
\begin{aligned}
I_{\lambda}\left(a, b ; q, q^{n}\right)= & \frac{1}{2^{n} Z_{n}(a, b ; q)} \\
& \times \operatorname{pf}_{1 \leqslant i, j \leqslant 2 n}\left(\frac{1}{2 \pi \mathrm{i}} \int_{\mathbb{T}}\left(z^{\lambda_{i}-\lambda_{j}-i+j}-z^{\lambda_{j}-\lambda_{i}+i-j}\right) \frac{z^{-1} \theta\left(z^{2} ; q\right)}{\left(a z^{ \pm 2}, b z^{ \pm 2} ; q^{2}\right)_{\infty}} \frac{\mathrm{d} z}{z}\right) .
\end{aligned}
$$

The $(i, j)$-entry of above pfaffian vanishes if $\lambda_{i}-\lambda_{j}-i+j$ is even. For the pfaffian to not vanish the set

$$
\left\{\lambda_{1}+2 n-1, \ldots, \lambda_{2 n-1}^{\prime}+1, \lambda_{2 n}\right\}
$$

must thus have $n$ even and $n$ odd elements. By Lemma 2.1 this is exactly the case if $\lambda$ has empty 2-core, which settles the non-vanishing part of the proposition.

For the second part, we take $a=q^{1-\alpha}$ and $b=q^{\alpha+1}$ with $\alpha \in\{0,1\}$ so that

$$
\frac{\theta\left(z^{2} ; q\right)}{\left(a z^{ \pm 2}, b z^{ \pm 2} ; q^{2}\right)_{\infty}^{2}}=\frac{\theta\left(q^{\alpha} z^{2} ; q^{2}\right)}{\theta\left(q^{\alpha+1} z^{2} ; q^{2}\right)_{\infty}}
$$

Assume now that $\lambda_{i}-\lambda_{j}-i+j$ is odd, say $2 k+1$. Then the $(i, j)$-entry of the pfaffian is given by

$$
\frac{1}{2 \pi \mathrm{i}} \int_{\mathbb{T}}\left(z^{2 k+1}-z^{-2 k-1}\right) \frac{z^{-1} \theta\left(q^{\alpha} z^{2} ; q^{2}\right)}{\theta\left(q^{\alpha+1} z^{2} ; q^{2}\right)} \frac{\mathrm{d} z}{z}
$$




$$
\begin{aligned}
& =\frac{1}{2 \pi \mathrm{i}} \sum_{i, j=0}^{\infty} \int_{\mathbb{T}}\left(z^{2 k+1}-z^{-2 k-1}\right) z^{2 i-2 j-1} q^{i+j+\alpha(i-j)} \frac{\left(1 / q ; q^{2}\right)_{i}\left(q ; q^{2}\right)_{j}}{\left(q^{2} ; q^{2}\right)_{i}\left(q^{2} ; q^{2}\right)_{j}} \frac{\mathrm{d} z}{z} \\
& =\sum_{i=0}^{\infty} q^{2 i-(\alpha-1) k} \frac{\left(1 / q ; q^{2}\right)_{i}\left(q ; q^{2}\right)_{i+k}}{\left(q^{2} ; q^{2}\right)_{i}\left(q^{2} ; q^{2}\right)_{i+k}}-\sum_{i=0}^{\infty} q^{2 i+(\alpha-1)(k+1)} \frac{\left(1 / q ; q^{2}\right)_{i}\left(q ; q^{2}\right)_{i-k-1}}{\left(q^{2} ; q^{2}\right)_{i}\left(q^{2} ; q^{2}\right)_{i-k-1}} \\
& =\frac{q^{-(\alpha-1) k}}{1-q^{2 k+1}} \frac{\left(q ; q^{2}\right)_{\infty}}{\left(q^{2} ; q^{2}\right)_{\infty}}-\frac{q^{(\alpha-1)(k+1)}}{1-q^{-2 k-1}} \frac{\left(q ; q^{2}\right)_{\infty}}{\left(q^{2} ; q^{2}\right)_{\infty}} \\
& =\frac{q^{-(\alpha-1) k}+q^{\alpha+(\alpha+1) k}}{1-q^{2 k+1}} \frac{\left(q ; q^{2}\right)_{\infty}^{2}}{\left(q^{2} ; q^{2}\right)_{\infty}^{2}}
\end{aligned}
$$

where the second equality follows from double use of the $q$-binomial theorem [13, equation (II.3)]

$$
{ }_{1} \phi_{0}(a ;-; q, z)=\frac{(a z ; q)_{\infty}}{(z ; q)_{\infty}}
$$

and the second-last equality from the $q$-Gauss sum [13, equation (II.8)]

$$
{ }_{2} \phi_{1}(a, b ; c ; q, z)=\frac{(c / a, c / b ; q)_{\infty}}{(c, c / a b ; q)_{\infty}} .
$$

Since

$$
Z_{n}\left(q^{1-\alpha}, q^{\alpha+1} ; q\right)=\frac{1+q^{\alpha n}}{2}\left(\frac{\left(q ; q^{2}\right)_{\infty}}{\left(q^{2} ; q^{2}\right)_{\infty}}\right)^{2 n} \prod_{i=1}^{n} \frac{\left(1-q^{2 i}\right)^{2 n-2 i}}{\left(1-q^{2 i-1}\right)^{2 n-2 i+1}},
$$

we obtain (9.5a) and (9.5b).

By [42, Proposition 4.9] the integrals (9.3a) and (9.3b) are equivalent to a pair of bounded Littlewood identities. To state these we define, for $\lambda$ a partition such that 2 -core $(\lambda)=0$,

$$
\begin{aligned}
& \kappa_{\lambda}^{(1)}(z ; q, t):=q^{n^{\mathrm{e}}\left(\lambda^{\prime}\right)-n^{\mathrm{o}}\left(\lambda^{\prime}\right)} t^{2 \hat{n}^{\mathrm{o}}(\lambda)-2 \hat{n}^{\mathrm{e}}(\lambda)}\left(\frac{q}{t}\right)^{|\lambda| / 2} \frac{C_{\lambda}^{0, \mathrm{e}}\left(z^{2} ; q, t\right)}{C_{\lambda}^{0, \mathrm{o}}\left(z^{2} q / t ; q, t\right)} \frac{C_{\lambda}^{-, \mathrm{e}}(t ; q, t)}{C_{\lambda}^{-, \mathrm{o}}(q ; q, t)}, \\
& \kappa_{\lambda}^{(2)}(z ; q, t):=\frac{q^{2 \hat{n}^{\mathrm{o}}\left(\lambda^{\prime}\right)-2 \hat{n}^{\mathrm{e}}\left(\lambda^{\prime}\right)} t^{n^{\mathrm{e}}(\lambda)-n^{\mathrm{o}}(\lambda)}+z q^{4 \hat{n}^{\mathrm{e}}\left(\lambda^{\prime}\right)-4 n^{\mathrm{o}}\left(\lambda^{\prime}\right)} t^{2 n^{\mathrm{o}}(\lambda)+3 \hat{n}^{\mathrm{o}}(\lambda)-5 \hat{n}^{\mathrm{e}}(\lambda)}}{1+z} \\
& \times\left(\frac{q}{t}\right)^{|\lambda| / 2} \frac{C_{\lambda}^{0, \mathrm{e}}\left(z^{2} ; q, t\right)}{C_{\lambda}^{0, \mathrm{o}}\left(z^{2} q / t ; q, t\right)} \frac{C_{\lambda}^{-, \mathrm{e}}(t ; q, t)}{C_{\lambda}^{-, \mathrm{o}}(q ; q, t)},
\end{aligned}
$$

and set $\kappa_{\lambda}^{(1)}(z ; q, t)=\kappa_{\lambda}^{(2)}(z ; q, t)=0$ if $\lambda$ has a non-trivial 2-core. As follows from Lemmas 2.2 and 2.3, in the non-vanishing case these two functions are related to $I_{\lambda}(q, t ; q, t, T)$ and $I_{\lambda}(1, q t ; q, t, T)$ as conjectured on the right-hand side of (9.3) via

$$
\kappa_{\lambda}^{(1)}(z ; q, t)=I_{\lambda^{\prime}}(t, q ; t, q, 1 / z) \quad \text { and } \quad \kappa_{\lambda}^{(2)}(z ; q, t)=I_{\lambda^{\prime}}(1, q t ; t, q, 1 / z) .
$$

Conjecture 9.4 (bounded Littlewood identities). For $m, n$ nonnegative integers,

$$
\sum_{\lambda \in P_{+}(n)} \kappa_{\lambda}^{(1)}\left(q^{-m} ; q, t\right) P_{\lambda}(x ; q, t)=\left(x_{1} \cdots x_{n}\right)^{m} K_{\left(m^{n}\right)}\left(x ; q, t ; q^{1 / 2},-q^{1 / 2}, t^{1 / 2},-t^{1 / 2}\right)
$$

and

$$
\sum_{\lambda \in P_{+}(n)} \kappa_{\lambda}^{(2)}\left(q^{-m} ; q, t\right) P_{\lambda}(x ; q, t)=\left(x_{1} \cdots x_{n}\right)^{m} K_{\left(m^{n}\right)}\left(x ; q, t ; 1,-1,(q t)^{1 / 2},-(q t)^{1 / 2}\right) .
$$


Both $\kappa_{\lambda}^{(1)}\left(q^{-m} ; q, t\right)$ and $\kappa_{\lambda}^{(2)}\left(q^{-m} ; q, t\right)$ vanish if $\lambda_{1}>2 m$ so that only partitions $\lambda$ contained in the rectangle $\left((2 m)^{n}\right)$ with 2 -core $(\lambda)=0$ contribute to the sum. We also remark that bounded Littlewood identities and integrals such as (9.2) satisfy a duality in which the Koornwinder parameters $t_{r}$ (for $0 \leqslant r \leqslant t_{3}$ ) are mapped to $-(q t)^{1 / 2} / t_{r}$, see [42]. With respect to this duality both vanishing integrals and bounded Littlewood identities are self-dual.

Finally, define

$$
\begin{aligned}
& \kappa_{\lambda}^{(1)}(q, t):=\lim _{z \rightarrow \infty} \kappa_{\lambda}^{(1)}(z ; q, t)=q^{2 \hat{n}^{\mathrm{o}}\left(\lambda^{\prime}\right)-2 \hat{n}^{\mathrm{e}}\left(\lambda^{\prime}\right)} t^{n^{\mathrm{e}}(\lambda)-n^{\mathrm{o}}(\lambda)} \frac{C_{\lambda}^{-, \mathrm{e}}(t ; q, t)}{C_{\lambda}^{-, \mathrm{o}}(q ; q, t)}, \\
& \kappa_{\lambda}^{(2)}(q, t):=\lim _{z \rightarrow \infty} \kappa_{\lambda}^{(2)}(z ; q, t)=q^{n^{\mathrm{e}}\left(\lambda^{\prime}\right)-n^{\mathrm{o}}\left(\lambda^{\prime}\right)} t^{2 \hat{n}^{\mathrm{o}}(\lambda)-2 \hat{n}^{\mathrm{e}}(\lambda)} \frac{C_{\lambda}^{-, \mathrm{e}}(t ; q, t)}{C_{\lambda}^{-, \mathrm{o}}(q ; q, t)} .
\end{aligned}
$$

In the large- $m, n$ limit, Conjecture 9.4 then simplifies to the following pair of unbounded Littlewood identities.

Conjecture 9.5 (Littlewood identities). We have

$$
\sum_{\lambda} \kappa_{\lambda}^{(1)}(q, t) P_{\lambda}(x ; q, t)=\prod_{i \geqslant 1} \frac{\left(t x_{i}^{2} ; q^{2}\right)_{\infty}}{\left(x_{i}^{2} ; q^{2}\right)_{\infty}} \prod_{i<j} \frac{\left(t x_{i} x_{j} ; q\right)_{\infty}}{\left(x_{i} x_{j} ; q\right)_{\infty}}
$$

and

$$
\sum_{\lambda} \kappa_{\lambda}^{(2)}(q, t) P_{\lambda}(x ; q, t)=\prod_{i \geqslant 1} \frac{\left(q t x_{i}^{2} ; q^{2}\right)_{\infty}}{\left(q x_{i}^{2} ; q^{2}\right)_{\infty}} \prod_{i<j} \frac{\left(t x_{i} x_{j} ; q\right)_{\infty}}{\left(x_{i} x_{j} ; q\right)_{\infty}} .
$$

The above identities are no longer self-dual and, as follows from Lemma 2.3 and (4.11) as well as Lemma 9.6 below, they form a dual pair with respect to $\omega_{q, t}$.

Lemma 9.6. Let $\omega_{q, t}$ be the automorphism of $\Lambda_{\mathbb{Q}(q, t)}$ given by (4.10). Then

$$
\omega_{q, t}\left(\prod_{i \geqslant 1} \frac{\left(t x_{i}^{2} ; q^{2}\right)_{\infty}}{\left(x_{i}^{2} ; q^{2}\right)_{\infty}} \prod_{i<j} \frac{\left(t x_{i} x_{j} ; q\right)_{\infty}}{\left(x_{i} x_{j} ; q\right)_{\infty}}\right)=\prod_{i \geqslant 1} \frac{\left(q t x_{i}^{2} ; t^{2}\right)_{\infty}}{\left(t x_{i}^{2} ; t^{2}\right)_{\infty}} \prod_{i<j} \frac{\left(q x_{i} x_{j} ; t\right)_{\infty}}{\left(x_{i} x_{j} ; t\right)_{\infty}} .
$$

Proof. We have

$$
\begin{aligned}
\mathcal{L}(a ; q, t):= & \prod_{i \geqslant 1} \frac{\left(a t x_{i}^{2} ; q^{2}\right)_{\infty}}{\left(a x_{i}^{2} ; q^{2}\right)_{\infty}} \prod_{i<j} \frac{\left(t x_{i} x_{j} ; q\right)_{\infty}}{\left(x_{i} x_{j} ; q\right)_{\infty}} \\
=\sum_{r \geqslant 0} & \left(\sum_{i \geqslant 1}\left(\log \left(1-q^{2 r} a t x_{i}^{2}\right)-\log \left(1-a q^{2 r} x_{i}^{2}\right)\right)\right. \\
& \left.+\sum_{i<j}\left(\log \left(1-q^{r} t x_{i} x_{j}\right)-\log \left(1-q^{r} x_{i} x_{j}\right)\right)\right) .
\end{aligned}
$$

Using $\log (1-x)=-\sum_{n \geqslant 1} x^{n} / n$, the sum over $r$ can be carried out by the geometric series. Since $\sum_{i<j}\left(x_{i} x_{j}\right)^{n}=\left(p_{n}^{2}-p_{2 n}\right) / 2$, we thus find

$$
\mathcal{L}(a ; q, t)=\sum_{n \geqslant 1} \frac{1-t^{n}}{n}\left(\frac{a^{n} p_{2 n}}{1-q^{2 n}}+\frac{1}{2} \frac{p_{n}^{2}-p_{2 n}}{1-q^{n}}\right) .
$$

In particular,

$$
\mathcal{L}(q ; q, t)=\frac{1}{2} \sum_{n \geqslant 1} \frac{1-t^{n}}{n}\left(\frac{p_{n}^{2}}{1-q^{n}}-\frac{p_{2 n}}{1+q^{n}}\right) .
$$


and

$$
\mathcal{L}(1 ; q, t)=\frac{1}{2} \sum_{n \geqslant 1} \frac{1-t^{n}}{n}\left(\frac{p_{n}^{2}}{1-q^{n}}+\frac{p_{2 n}}{1+q^{n}}\right) .
$$

Applying $\omega_{q, t}$ to this last expression yields

$$
\mathcal{L}(1 ; q, t)=\frac{1}{2} \sum_{n \geqslant 1} \frac{1-q^{n}}{n}\left(\frac{p_{n}^{2}}{1-t^{n}}-\frac{p_{2 n}}{1+t^{n}}\right)=\mathcal{L}(t ; t, q),
$$

completing the proof.

\section{Postscript}

One of the referees posed the question as to how the branching rule (1.12a) compares to conjectural branching formulas of Hoshino and Shiraishi [15] between certain type A and C asymptotically-free eigenfunctions of Macdonald operators. In this postscript we address the referee's question.

Hoshino and Shiraishi [15, Section 9] considered functions

$$
\tilde{\varphi}^{\left(\mathrm{A}_{2 n-1}\right)}(s|x| q, t) \quad \text { and } \quad \varphi^{\left(\mathrm{C}_{n}\right)}(s|x| q, t),
$$

where $s=\left(s_{1}, \ldots, s_{n}\right)$ and $x=\left(x_{1}, \ldots, x_{n}\right)$, such that for $\lambda \in P_{+}(n)$

$$
\begin{aligned}
& x^{\lambda} \tilde{\varphi}^{\left(\mathrm{A}_{2 n-1}\right)}\left(t^{n} q^{\lambda_{1}}, \ldots, t q^{\lambda_{n}}|x| q, t\right)=P_{\lambda}\left(x^{ \pm} ; q, t\right), \\
& x^{\lambda} \varphi^{\left(\mathrm{C}_{n}\right)}\left(t^{n} q^{\lambda_{1}}, \ldots, t q^{\lambda_{n}}|x| q, t\right)=P_{\lambda}^{\left(\mathrm{C}_{n}, \mathrm{C}_{n}\right)}(x ; q, t, t),
\end{aligned}
$$

where $x^{\lambda}:=x_{1}^{\lambda_{1}} \cdots x_{n}^{\lambda_{n}}$. Here we note that the function $\tilde{\varphi}^{\left(\mathrm{A}_{2 n-1}\right)}$ arises by folding the asymptotically-free solution of the trigonometric Ruijsenaars model [30, 48] of rank $2 n-1$, see [15] for more details. In the rank-one case,

$$
\tilde{\varphi}^{\left(\mathrm{A}_{1}\right)}\left(s_{1}\left|x_{1}\right| q, t\right)=\varphi^{\left(\mathrm{C}_{1}\right)}\left(s_{1}\left|x_{1}\right| q, t\right)={ }_{2} \phi_{1}\left[\begin{array}{c}
t, t s_{1} \\
q s_{1}
\end{array} ; q, \frac{q x_{1}^{2}}{t}\right] .
$$

Let $\theta=\left\{\theta_{i j}\right\}_{1 \leqslant i<j \leqslant n}$ be a set of nonnegative integers and, for $1 \leqslant i \leqslant n$, define

$$
\phi_{i}:=\sum_{j=1}^{i-1} \theta_{j i}+\sum_{j=i+1}^{n} \theta_{i j}
$$

and $\phi:=\left(\phi_{1}, \ldots, \phi_{n}\right)$. Hoshino and Shiraishi considered the problem of determining the rational functions $e_{n}(s ; \theta ; q, t)$ for $n \geqslant 1$, such that the following branching rule holds:

$$
\tilde{\varphi}^{\left(\mathrm{A}_{2 n-1}\right)}(s|x| q, t)=\sum_{\theta} e_{n}(s ; \theta ; q, t) \varphi^{\left(\mathrm{C}_{n}\right)}\left(s q^{-\phi}|x| q, t\right) x^{-\phi}
$$

where $s q^{-\phi}:=\left(s_{1} q^{-\phi_{1}}, \ldots, s_{n} q^{-\phi_{n}}\right)$ and $x^{-\phi}:=x_{1}^{-\phi_{1}} \cdots x_{n}^{-\phi_{n}}=\prod_{1 \leqslant i<j \leqslant n}\left(x_{i} x_{j}\right)^{-\theta_{i j}}$.

By (9.8), $e_{1}\left(s_{1} ;-; q, t\right)=1$. In [15, Conjectures 9.12 and 9.13] Hoshino and Shiraishi made the following two conjectures.

Conjecture 9.7. We have

$$
e_{2}\left(s_{1}, s_{2} ; \theta_{12} ; q, t\right)=\frac{\left(t, t / s_{1}, t / s_{2}, q^{\theta_{12}+1} / t s_{1} s_{2} ; q\right)_{\theta_{12}}}{\left(q, q / s_{1}, q / s_{2}, q^{\theta_{12} / s_{1}} s_{2} ; q\right)_{\theta_{12}}}\left(\frac{q}{t}\right)^{\theta_{12}}
$$


and

$$
\begin{aligned}
& e_{3}\left(s_{1}, s_{2}, s_{3} ; \theta_{12}, \theta_{13}, \theta_{23} ; q, t\right)=\prod_{i=1}^{3} \frac{\left(t / s_{i} ; q\right)_{\phi_{i}}}{\left(q / s_{i} ; q\right)_{\phi_{i}}} \\
& \quad \times \prod_{1 \leqslant i<j \leqslant 3} \frac{\left(t s_{j} / s_{i}, q^{1-\theta_{j, 6-i-j}} s_{j} / t s_{i} ; q\right)_{\theta_{1,5-j}}}{\left(q s_{j} / s_{i}, q^{-\theta_{j, 6-i-j}} s_{j} / s_{i} ; q\right)_{\theta_{1,5-j}}} \frac{\left(t, q^{1+\sum_{1 \leqslant k<l \leqslant 3} \theta_{k l}} / t s_{i} s_{j} ; q\right)_{\theta_{i j}}}{\left(q, q^{\left.\sum_{1 \leqslant k<l \leqslant 3} \theta_{k l} / s_{i} s_{j} ; q\right)_{\theta_{i j}}}\left(\frac{q}{t}\right)^{\theta_{i j}},\right.},
\end{aligned}
$$

where $\theta_{j i}:=\theta_{i j}$ for $1 \leqslant i<j \leqslant 3$.

To now answer the question of the referee, we first notice that (9.7b) can be used to define $P_{\lambda}^{\left(\mathrm{C}_{n}, \mathrm{C}_{n}\right)}(x ; q, t, t)$ for integer sequences $\lambda=\left(\lambda_{1}, \ldots, \lambda_{n}\right)$ that are not necessarily partitions. In (9.9) we can then specialise $s_{i}=q^{\lambda_{i}} t^{n-i+1}$ for $1 \leqslant i \leqslant n$, multiply both sides by $x^{\lambda}$, and use $(9.7 \mathrm{a})$ and $(9.7 \mathrm{~b})$, to obtain

$$
P_{\lambda}\left(x^{ \pm} ; q, t\right)=\sum_{\theta} e_{n}\left(q^{\lambda_{1}} t^{n}, \ldots, q^{\lambda_{n}} t ; \theta ; q, t\right) P_{\left(\lambda_{1}-\phi_{1}, \ldots, \lambda_{n}-\phi_{n}\right)}^{\left(\mathrm{C}_{n}, \mathrm{C}_{n}\right)}(x ; q, t, t) .
$$

Assuming Conjecture 9.7,

$$
e_{2}\left(q^{\lambda_{1}} t^{2}, q^{\lambda_{2}} t ; \theta_{12} ; q, t\right)=0
$$

unless $\theta_{12} \leqslant \lambda_{2}$, and

$$
e_{3}\left(q^{\lambda_{1}} t^{3}, q^{\lambda_{2}} t^{2}, q^{\lambda_{3}} t ; \theta_{12}, \theta_{13}, \theta_{23} ; q, t\right)=0
$$

unless $\theta_{12} \leqslant \lambda_{2}-\lambda_{3}, \theta_{13} \leqslant \lambda_{1}-\lambda_{2}$ and $\theta_{13}+\theta_{23} \leqslant \lambda_{3}$. For $n=2$ this implies that $\lambda_{1}-\phi_{1}=$ $\lambda_{1}-\theta_{12} \geqslant 0$, and for $n=3$ it implies

$$
\begin{aligned}
& \left(\lambda_{1}-\phi_{1}\right)-\left(\lambda_{2}-\phi_{2}\right)=\lambda_{1}-\lambda_{2}-\theta_{13}+\theta_{23} \geqslant \theta_{23} \geqslant 0 \\
& \left(\lambda_{2}-\phi_{2}\right)-\left(\lambda_{3}-\phi_{3}\right)=\lambda_{2}-\lambda_{3}-\theta_{12}+\theta_{13} \geqslant \theta_{13} \geqslant 0 \\
& \lambda_{3}-\phi_{3}=\lambda_{3}-\theta_{13}-\theta_{23} \geqslant 0 .
\end{aligned}
$$

It is thus reasonable to conjecture that

$$
e_{n}\left(q^{\lambda_{1}} t^{n}, \ldots, q^{\lambda_{n}} t ; \theta ; q, t\right)=0
$$

if $\left(\lambda_{1}-\phi_{1}, \ldots, \lambda_{n}-\phi_{n}\right) \notin P_{+}(n)$, so that

$$
P_{\lambda}\left(x^{ \pm} ; q, t\right)=\sum_{\substack{\theta \\\left(\lambda_{1}-\phi_{1}, \ldots, \lambda_{n}-\phi_{n}\right) \in P_{+}(n)}} e_{n}\left(q^{\lambda_{1}} t^{n}, \ldots, q^{\lambda_{n}} t ; \theta ; q, t\right) P_{\left(\lambda_{1}-\phi_{1}, \ldots, \lambda_{n}-\phi_{n}\right)}^{\left(\mathrm{C}_{n}, \mathrm{C}_{n}\right)}(x ; q, t, t) .
$$

If $\lambda=\left(m^{r}\right)$ for $0 \leqslant r \leqslant n$ we may compare the above with the branching rule (1.12a). Let $\rho:=\lfloor r / 2\rfloor, I_{n}:=\{(i, j): 1 \leqslant i<j \leqslant n\}$,

$$
J_{r}:=\{(r-2 i+1, r-2 i+2): 1 \leqslant i \leqslant \rho\} \quad \text { and } \quad \bar{J}_{r, n}:=I_{n} \backslash J_{r} .
$$

It then follows from (1.12a) that

$$
e_{n}\left(q^{m} t^{n}, \ldots, q^{m} t^{n-r+1}, t^{n-r}, \ldots, t ; \theta ; q, t\right)
$$

is non-vanishing if and only if

$$
\begin{aligned}
& \theta_{i j}=0 \quad \text { for }(i, j) \in \bar{J}_{n, r}, \\
& m \geqslant \theta_{r-1, r} \geqslant \theta_{r-3, r-2} \geqslant \cdots \geqslant \theta_{r-2 \rho+1, r-2 \rho+2} \geqslant 0,
\end{aligned}
$$


in which case

$$
\begin{array}{ll}
\phi_{r-2 i+1}=\phi_{2-2 i+2}=\theta_{r-2 i+1,2 i-2 i+2} & \text { for } 1 \leqslant i \leqslant \rho, \\
\phi_{i}=0 & \text { otherwise. }
\end{array}
$$

Moreover, for such non-vanishing $\theta$ we have the following identification of $e_{n}$ and the function $c_{\lambda^{2}}$ defined in (1.9a). Let $\mu \subset\left(m^{r}\right)$ such that $\mu^{\prime}$ is even (i.e., $\left.\mu=\lambda^{2}\right)$ and let $\theta$ be fixed in terms of $\mu($ or $\lambda)$ as

$$
\begin{array}{ll}
\theta_{r-2 i+1, r-2 i+2}=\mu_{2 i-1}=\lambda_{i} & \text { for } 1 \leqslant i \leqslant \rho, \\
\theta_{i j}=0 & \text { for }(i, j) \in \bar{J}_{n, r} .
\end{array}
$$

Then

$$
e_{n}\left(q^{m} t^{n}, \ldots, q^{m} t^{n-r+1}, t^{n-r}, \ldots, t ; \theta ; q, t\right)=c_{\lambda^{2}}\left(q^{-m}, t^{-(n-r)} ; q, t\right) .
$$

It is readily checked that the rational functions $e_{2}$ and $e_{3}$ of Conjecture 9.7 satisfy (9.10), showing that the conjecture is consistent with the branching rule (1.12a). In particular, we have the slightly more general

$$
\begin{aligned}
& e_{2}\left(t^{2} s, t ; \theta_{12} ; q, t\right)=\delta_{\theta_{12}, 0}, \\
& e_{2}\left(t^{2} s, t s ; \theta_{12} ; q, t\right)=c_{\left(\theta_{12}, \theta_{12}\right)}(1 / s, 1 ; q, t)
\end{aligned}
$$

and

$$
\begin{aligned}
& e_{3}\left(t^{3} s, t^{2}, t ; \theta_{12}, \theta_{13}, \theta_{23} ; q, t\right)=\delta_{\theta_{12}, 0} \delta_{\theta_{13}, 0} \delta_{\theta_{23}, 0} \\
& e_{3}\left(t^{3} s, t^{2} s, t ; \theta_{12}, \theta_{13}, \theta_{23} ; q, t\right)=c_{\left(\theta_{12}, \theta_{12}\right)}(1 / s, 1 / t ; q, t) \delta_{\theta_{13}, 0} \delta_{\theta_{23}, 0} \\
& e_{3}\left(t^{3} s, t^{2} s, t s ; \theta_{12}, \theta_{13}, \theta_{23} ; q, t\right)=c_{\left(\theta_{23}, \theta_{23}\right)}(1 / s, 1 ; q, t) \delta_{\theta_{12}, 0} \delta_{\theta_{13}, 0}
\end{aligned}
$$

\section{Acknowledgements}

We thank one of the referees of our paper for suggesting we compare the branching rule (1.12a) with [15, Conjectures 9.12 and 9.13] by Hoshino and Shiraishi. This work was supported by the Australian Research Council Discovery Grant DP170102648 and a KIAS Individual Grant (MG067302) at Korea Institute for Advanced Study.

\section{References}

[1] Albion S.P., Rains E.M., Warnaar S.O., AFLT-type Selberg integrals, arXiv:2001.05637.

[2] Andrews G.E., On q-analogues of the Watson and Whipple summations, SIAM J. Math. Anal. 7 (1976), $332-336$.

[3] Baker T.H., Forrester P.J., Transformation formulas for multivariable basic hypergeometric series, Methods Appl. Anal. 6 (1999), 147-164, arXiv:math.QA/9803146.

[4] Bressoud D., Ismail M.E.H., Stanton D., Change of base in Bailey pairs, Ramanujan J. 4 (2000), 435-453, arXiv:math.CO/9909053.

[5] Coskun H., Gustafson R.A., Well-poised Macdonald functions $W_{\lambda}$ and Jackson coefficients $\omega_{\lambda}$ on $B C_{n}$, in Jack, Hall-Littlewood and Macdonald polynomials, Contemp. Math., Vol. 417, Amer. Math. Soc., Providence, RI, 2006, 127-155, arXiv:math.CO/0412153.

[6] de Bruijn N.G., On some multiple integrals involving determinants, J. Indian Math. Soc. (N.S.) 19 (1955), $133-151$.

[7] van Diejen J.F., Emsiz E., Pieri formulas for Macdonald's spherical functions and polynomials, Math. Z. 269 (2011), 281-292, arXiv:1009.4482. 
[8] van Diejen J.F., Emsiz E., Branching formula for Macdonald-Koornwinder polynomials, J. Algebra 444 (2015), 606-614, arXiv:1408.2280.

[9] van Diejen J.F., Emsiz E., Branching rules for symmetric hypergeometric polynomials, in Representation Theory, Special Functions and Painlevé Equations - RIMS 2015, Adv. Stud. Pure Math., Vol. 76, Math. Soc. Japan, Tokyo, 2018, 125-153, arXiv:1601.06186.

[10] van Diejen J.F., Spiridonov V.P., An elliptic Macdonald-Morris conjecture and multiple modular hypergeometric sums, Math. Res. Lett. 7 (2000), 729-746.

[11] van Diejen J.F., Spiridonov V.P., Elliptic Selberg integrals, Int. Math. Res. Not. 2001 (2001), $1083-1110$.

[12] Frenkel I.B., Turaev V.G., Elliptic solutions of the Yang-Baxter equation and modular hypergeometric functions, in The Arnold-Gelfand Mathematical Seminars, Birkhäuser Boston, Boston, MA, 1997, 171-204.

[13] Gasper G., Rahman M., Basic hypergeometric series, 2nd ed., Encyclopedia of Mathematics and its Applications, Vol. 96, Cambridge University Press, Cambridge, 2004.

[14] Gustafson R.A., A generalization of Selberg's beta integral, Bull. Amer. Math. Soc. (N.S.) 22 (1990), 97-105.

[15] Hoshino A., Shiraishi J., Macdonald polynomials of type $C_{n}$ with one-column diagrams and deformed Catalan numbers, SIGMA 14 (2018), 101, 33 pages, arXiv:1801.09939.

[16] Hoshino A., Shiraishi J., Branching rules for Koornwinder polynomials with one column diagrams and matrix inversions, SIGMA 16 (2020), 084, 28 pages, arXiv:2002.02148.

[17] Ito M., Noumi M., Derivation of a $B C_{n}$ elliptic summation formula via the fundamental invariants, Constr. Approx. 45 (2017), 33-46, arXiv:1504.07108.

[18] King R.C., Branching rules for classical Lie groups using tensor and spinor methods, J. Phys. A: Math. Gen. 8 (1975), 429-449.

[19] Koike K., Terada I., Young-diagrammatic methods for the representation theory of the classical groups of type $B_{n}, C_{n}, D_{n}$, J. Algebra 107 (1987), 466-511.

[20] Koornwinder T.H., Askey-Wilson polynomials for root systems of type $B C$, in Hypergeometric Functions on Domains of Positivity, Jack Polynomials, and Applications (Tampa, FL, 1991), Contemp. Math., Vol. 138, Amer. Math. Soc., Providence, RI, 1992, 189-204.

[21] Krattenthaler C., Identities for classical group characters of nearly rectangular shape, J. Algebra 209 (1998), 1-64, arXiv:math.RT/9808118.

[22] Kwon J.H., Combinatorial extension of stable branching rules for classical groups, Trans. Amer. Math. Soc. 370 (2018), 6125-6152, arXiv:1512.01877.

[23] Lam T., Lapointe L., Morse J., Shimozono M., The poset of $k$-shapes and branching rules for $k$-Schur functions, Mem. Amer. Math. Soc. 223 (2013), vi+101 pages.

[24] Lascoux A., Warnaar S.O., Branching rules for symmetric functions and $\mathfrak{s l}_{n}$ basic hypergeometric series, Adv. in Appl. Math. 46 (2011), 424-456, arXiv:0903.3996.

[25] Littlewood D.E., The theory of group characters and matrix representations of groups, Oxford University Press, New York, 1940.

[26] Macdonald I.G., Symmetric functions and Hall polynomials, 2nd ed., Oxford Mathematical Monographs, The Clarendon Press, Oxford University Press, New York, 1995.

[27] Macdonald I.G., Orthogonal polynomials associated with root systems, Sém. Lothar. Combin. 45 (2000), Art. B45a, 40 pages, arXiv:math.QA/0011046.

[28] Mimachi K., A duality of MacDonald-Koornwinder polynomials and its application to integral representations, Duke Math. J. 107 (2001), 265-281.

[29] Naito S., Sagaki D., An approach to the branching rule from $\mathfrak{s l}_{2 n}(\mathbb{C})$ to $\mathfrak{s p}_{2 n}(\mathbb{C})$ via Littelmann's path model, J. Algebra 286 (2005), 187-212.

[30] Noumi M., Shiraishi J., A direct approach to the bispectral problem for the Ruijsenaars-Macdonald $q$ difference operators, arXiv:1206.5364.

[31] Okada S., Applications of minor summation formulas to rectangular-shaped representations of classical groups, J. Algebra 205 (1998), 337-367.

[32] Okounkov A., BC-type interpolation Macdonald polynomials and binomial formula for Koornwinder polynomials, Transform. Groups 3 (1998), 181-207, arXiv:q-alg/9611011.

[33] Okounkov A., Olshanski G., Shifted Schur functions. II. The binomial formula for characters of classical groups and its applications, in Kirillov's Seminar on Representation Theory, Amer. Math. Soc. Transl. Ser. 2, Vol. 181, Amer. Math. Soc., Providence, RI, 1998, 245-271. 
[34] Proctor R.A., Shifted plane partitions of trapezoidal shape, Proc. Amer. Math. Soc. 89 (1983), 553-559.

[35] Rains E.M., BC ${ }_{n}$-symmetric polynomials, Transform. Groups 10 (2005), 63-132, arXiv:math.QA/0112035.

[36] Rains E.M., $B C_{n}$-symmetric Abelian functions, Duke Math. J. 135 (2006), 99-180, arXiv:math.CO/0402113.

[37] Rains E.M., Limits of elliptic hypergeometric integrals, Ramanujan J. 18 (2009), 257-306, arXiv:math.CA/0607093.

[38] Rains E.M., Transformations of elliptic hypergeometric integrals, Ann. of Math. 171 (2010), 169-243, arXiv:math.QA/0309252.

[39] Rains E.M., Elliptic Littlewood identities, J. Combin. Theory Ser. A 119 (2012), 1558-1609, arXiv:0806.0871.

[40] Rains E.M., Multivariate quadratic transformations and the interpolation kernel, SIGMA 14 (2018), 019, 69 pages, arXiv:1408.0305.

[41] Rains E.M., Vazirani M., Vanishing integrals of Macdonald and Koornwinder polynomials, Transform. Groups 12 (2007), 725-759.

[42] Rains E.M., Warnaar S.O., Bounded Littlewood identities, Mem. Amer. Math. Soc., to appear, arXiv:1506.02755.

[43] Rosengren H., A proof of a multivariable elliptic summation formula conjectured by Warnaar, in $q$-series with applications to combinatorics, number theory, and physics (Urbana, IL, 2000), Contemp. Math., Vol. 291, Amer. Math. Soc., Providence, RI, 2001, 193-202, arXiv:math.CA/0101073.

[44] Rosengren H., Schlosser M.J., Multidimensional matrix inversions and elliptic hypergeometric series on root systems, SIGMA 16 (2020), 088, 21 pages, arXiv:2005.02203.

[45] Rosengren H., Warnaar S.O., Elliptic hypergeometric functions associated with root systems, in Encyclopedia of Special Functions: The Askey-Bateman Project, Vol. 2, Multivariable Special Functions, Cambridge University Press, Cambridge, 2020, 159-186, arXiv:1704.08406.

[46] Ruijsenaars S.N.M., First order analytic difference equations and integrable quantum systems, J. Math. Phys. 38 (1997), 1069-1146.

[47] Schumann B., Torres J., A non-Levi branching rule in terms of Littelmann paths, Proc. Lond. Math. Soc. 117 (2018), 1077-1100, arXiv:1607.08225.

[48] Shiraishi J., A conjecture about raising operators for Macdonald polynomials, Lett. Math. Phys. 73 (2005), 71-81, arXiv:math.QA/0503727.

[49] Spiridonov V.P., On the elliptic beta function, Russian Math. Surveys 56 (2001), 185-186.

[50] Spiridonov V.P., Theta hypergeometric series, in Asymptotic Combinatorics with Application to Mathematical Physics (St. Petersburg, 2001), NATO Sci. Ser. II Math. Phys. Chem., Vol. 77, Kluwer Acad. Publ., Dordrecht, 2002, 307-327, arXiv:math.CA/0303204.

[51] Stanley R.P., Enumerative combinatorics, Vol. 2, Cambridge Studies in Advanced Mathematics, Vol. 62, Cambridge University Press, Cambridge, 1999.

[52] Warnaar S.O., Summation and transformation formulas for elliptic hypergeometric series, Constr. Approx. 18 (2002), 479-502, arXiv:math.QA/0001006.

[53] Warnaar S.O., Extensions of the well-poised and elliptic well-poised Bailey lemma, Indag. Math. (N.S.) 14 (2003), 571-588, arXiv:math.CA/0309241.

[54] Warnaar S.O., Summation formulae for elliptic hypergeometric series, Proc. Amer. Math. Soc. 133 (2005), 519-527, arXiv:math.CA/0309242. 